\title{
Dilepton and photon production in the presence of a nontrivial Polyakov loop
}

\author{
Yoshimasa Hidaka, ${ }^{a}$ Shu Lin, ${ }^{b}$ Robert D. Pisarski ${ }^{c, b}$ and Daisuke Satow ${ }^{d}$ \\ ${ }^{a}$ Theoretical Research Division, Nishina Center, RIKEN, \\ Wako 351-0198, Japan \\ ${ }^{b}$ RIKEN/BNL Research Center, Brookhaven National Laboratory, \\ Upton, NY 11973, U.S.A. \\ ${ }^{c}$ Department of Physics, Brookhaven National Laboratory, \\ Upton, NY 11973, U.S.A. \\ ${ }^{d}$ European Centre for Theoretical Studies in Nuclear Physics and Related Areas (ECT*), \\ and Fondazione Bruno Kessler, \\ Villa Tambosi, Strada delle Tabarelle 286, I-38123 Villazzano (TN), Italy \\ E-mail: hidaka@riken.jp, slin@quark.phy.bnl.gov, pisarski@bnl.gov, \\ daisuke.sato@riken.jp
}

ABstract: We calculate the production of dileptons and photons in the presence of a nontrivial Polyakov loop in QCD. This is applicable to the semi-Quark Gluon Plasma (QGP), at temperatures above but near the critical temperature for deconfinement. The Polyakov loop is small in the semi-QGP, and near unity in the perturbative QGP. Working to leading order in the coupling constant of QCD, we find that there is a mild enhancement, $\sim 20 \%$, for dilepton production in the semi-QGP over that in the perturbative QGP. In contrast, we find that photon production is strongly suppressed in the semi-QGP, by about an order of magnitude, relative to the perturbative QGP. In the perturbative QGP photon production contains contributions from $2 \rightarrow 2$ scattering and collinear emission with the Landau-Pomeranchuk-Migdal (LPM) effect. In the semi-QGP we show that the two contributions are modified differently. The rate for $2 \rightarrow 2$ scattering is suppressed by a factor which depends upon the Polyakov loop. In contrast, in an $\mathrm{SU}(N)$ gauge theory the collinear rate is suppressed by $1 / N$, so that the LPM effect vanishes at $N=\infty$. To leading order in the semi-QGP at large $N$, we compute the rate from $2 \rightarrow 2$ scattering to the leading logarithmic order and the collinear rate to leading order.

KeYwords: QCD Phenomenology, Heavy Ion Phenomenology

ArXiv EPrint: 1504.01770 


\section{Contents}

1 Introduction $\quad 2$

2 Semi-quark gluon plasma $\quad 3$

2.1 Double line notation 3

2.2 The Polyakov loop in Euclidean spacetime 3

2.3 Analytic continuation to Minkowski space-time 5

2.4 Relation to lattice results 6

3 Dilepton production rate $\quad 8$

3.1 Computation to leading order 8

3.2 Enhancement of dilepton production in the confined phase versus the perturbative Quark-Gluon Plasma 11

4 Photon production $\quad 14$

4.1 Overview 14

$\begin{array}{lll}\text { 4.2 Hard momentum exchange with trivial Polyakov loop } & 15\end{array}$

4.3 Hard momentum exchange with nontrivial Polyakov loop 20

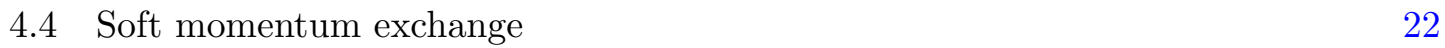

4.5 Why so few photons are produced in the semi-QGP 28

5 Collinear rate $\quad 31$

$\begin{array}{lll}5.1 & \text { Review of AMY's calculation of photon production } & 31\end{array}$

5.1.1 Photon self-energy in RA basis 31

5.1.2 Reduction of spinor structure 33

5.1.3 Color structure in the double line basis $\quad 35$

5.1.4 Resummation of infinite self-energy diagrams 35

$\begin{array}{ll}\text { 5.2 Photon self-energy with nontrivial Polyakov loop } & 38\end{array}$

5.2.1 Quark and gluon thermal masses with background color charge 38

5.2.2 AMY's integral equation with one color index 40

5.3 Photon rate in the collinear regime at large N 43

5.4 Why the LPM effect is suppressed in the semi-QGP 46

$\begin{array}{lll}6 & \text { Summary and concluding remarks } & 47\end{array}$

A Corrections to Boltzmann approximation to thermal distribution $\begin{array}{ll}\text { functions } & 48\end{array}$

B Thermal gluon mass in the presence of Polyakov loop 49 


\section{Introduction}

In many ways, the collisions of heavy ions at high energies appear to be well described by thermal properties of a Quark-Gluon Plasma (QGP). Certainly the bulk properties of hadrons are accurately modeled by a nearly ideal plasma, using hydrodynamics [1-5].

It is also important to consider electromagnetic probes of a QGP such as dilepton [6-25] and photon [26-43] production. Theoretically, these can be computed in the (resummed) perturbative QGP at high temperature [44], by using hadronic models at low temperature [45, 46], and using the AdS/CFT correspondence [47-49].

Neither applies directly to Quantum ChromoDynamics (QCD) at temperatures near that for the phase transition, at a temperature $T_{c}$. There are various models which can be used to study the region near $T_{c}$, which we call the "semi" Quark-Gluon Plasma. This includes Parton Hadron String Dynamics [50-55], Polyakov loop models [56-58], the functional renormalization group [59-70], background field methods [71-73], and lastly, matrix models [74-85].

Experiment has discovered several unexpected phenomena. For dileptons, there is an enhancement at invariant masses below that for the $\rho$-meson. This is observed from energies at the Super Proton Synchrotron (SPS) at CERN, to the Relativistic Heavy Ion Collider (RHIC) at Brookhaven National Laboratory, and onto the Large Hadron Collider (LHC) at CERN [24].

Another puzzle appears in the photon spectrum: there is an unexpectedly large elliptic flow for photons at moderate momenta, comparable to the elliptical flow observed for hadrons $[86,87]$. This large elliptic flow for photons is very difficult to understand from either a perturbative analysis or from AdS/CFT.

In this paper we consider electromagnetic signals in a matrix model of the semi-QGP. In a matrix model the relevant parameter is the expectation value of the Polyakov loop: properly normalized, the expectation value of the loop is near unity in the perturbative QGP [88-90]. Numerical simulations on the lattice [91-97] find that for QCD, there is no true phase transition, only a rapid increase in the number of degrees of freedom. For our purposes, whether or not there is a true phase transition is irrelevant: all that matters is that the (renormalized) Polyakov loop, which from the lattice is $\langle\ell\rangle \sim 0.1$ at $T_{c}$, is small [91-97].

A brief summary of the results of this analysis has appeared previously [98]. In this paper we describe the computations in full. These are straightforward, simply a matter of computing in the presence of a background field for the time-like component of the gluon vector potential, $A_{0}$. We then compute to leading order in the QCD coupling $g$. These formalisms will be explained in section 2 . For photons, we only compute to leading logarithmic order, which means that we regard the logarithm of some large number as much larger than unity.

In the semi-QGP, the production of colored particles is suppressed by powers of the Polyakov loop as $T \rightarrow T_{c}$. This is natural, as in the pure gauge theory, there are no colored particles in the confined phase. Thus one might expect that dilepton production is suppressed in the semi-QGP, relative to that in the perturbative phase. We make this 
comparison at the same temperature, and the same value of the QCD coupling, so that the ratio is only a function of the value of the Polyakov loop in section 3. In contrast to the naive expectation above, we find a mild enhancement of dilepton production in the semi-QGP, even into the confined phase. This is because for an off-shell photon, it can proceed directly through a color singlet channel, a quark anti-quark pair. While a single quark or anti-quark is suppressed by a power of the Polyakov loop, a quark anti-quark pair is not. We also show that to leading order, a Polyakov Nambu-Jona-Lasino model [56-58] gives essentially the same result for dilepton production [99] as our matrix model. As we discuss, this equality is not true beyond leading order.

The production of real photons, which will be analyzed in section 4.1, is very different. Kinematically, a photon on its mass shell cannot decay directly into a quark anti-quark pair. Therefore, the leading contribution is from a 2 to 2 scattering, which includes the Compton scattering of a quark with a gluon and the pair annihilation of a quark anti-quark pair. These particles also can form a color singlet like the case of the dilepton production, but for an $\mathrm{SU}(N)$ gauge theory, the ratio of the color singlet state to the number of all the states is suppressed by $1 / N^{2}$ at large $N$. Consequently, we find a strong suppression of real photon production in the semi-QGP. The contribution from the collinear emission of the photon, which also can contribute at the leading order to the photon production, is discussed in section 5 .

\section{Semi-quark gluon plasma}

\subsection{Double line notation}

It is useful to compute the color factors using the double line basis [76]. In this basis, as usual fundamental quarks carry a single index in the fundamental representation, $a=$ $1, \cdots, N$. Gluons, however, carry a pair of fundamental indices, $(a b)$. For an $\operatorname{SU}(N)$ group there are $N^{2}$ such pairs, and so this basis is overcomplete by one generator. This is compensated by introducing the operator

$$
\mathcal{P}_{c d}^{a b}=\delta_{c}^{a} \delta_{d}^{b}-\frac{1}{N} \delta^{a b} \delta_{c d} .
$$

This is a projection operator,

$$
\mathcal{P}_{e f}^{a b} \mathcal{P}_{c d}^{e f}=\mathcal{P}_{c d}^{a b} .
$$

In the double line basis, the vertex between a quark anti-quark pair and a gluon is proportional to this projection operator,

$$
\left(T^{a b}\right)_{c d}=\frac{1}{\sqrt{2}} \mathcal{P}_{c d}^{a b}
$$

The other vertices are not relevant for the present discussion.

\subsection{The Polyakov loop in Euclidean spacetime}

To introduce the effect of nontrivial Polyakov loop in perturbative calculation, we work in an effective model introduced in ref. [76]. The Lagrangian of that model is the same as 
that in QCD with $N$ colors, but in a mean field type approximation, we take the temporal component of the gluon field to be a constant, diagonal matrix,

$$
A_{0}^{a b}=\frac{1}{g} \delta^{a b} Q^{a}
$$

where summation over index $a$ is not taken and $g$ is the coupling constant. There is no background field for the spatial components of the gluon, $A_{i}$. As the gauge group is $\operatorname{SU}(N)$, $A_{0}$ is traceless, and the sum of the $Q$ 's vanishes, $\sum_{a} Q^{a}=0$.

The Wilson line in the temporal direction is

$$
\mathbf{L}(\vec{x}) \equiv \mathcal{P} \exp \left(i g \int_{0}^{1 / T} d \tau A_{0}(\tau, \vec{x})\right),
$$

where $\mathcal{P}$ denotes path ordering and $\tau$ is the imaginary time, $\tau: 0 \rightarrow 1 / T$.

To leading order in the coupling constant, a mean field approximation implies that we can neglect fluctuations in $A_{0}$. The variable $Q$ is naturally proportional to the temperature, so it is useful to introduce a dimensionless variable $q$, where

$$
Q^{a}=2 \pi T q^{a} .
$$

In this paper we shall use both the $Q^{a}$ 's and the $q^{a}$ 's. For intermediate expressions the $Q^{a}$ 's are more convenient, but final expressions are simpler in terms of the $q^{a}$ 's.

Traces of powers of the the Wilson line are Polyakov loops,

$$
\ell_{n}(Q) \equiv \frac{1}{N}\left\langle\operatorname{tr} \mathbf{L}^{n}\right\rangle=\frac{1}{N} \sum_{a=1}^{N} e^{i n Q^{a} / T},
$$

and are gauge invariant. Since it arises frequently we write a loop without the subscript as the first Polyakov loop, $\ell=\ell_{1}$.

In general there are $N-1$ independent $Q^{a}$ 's. For the problems of interest in this paper, though, we can perform a global color rotation to enforce that the expectation value of the loop $\ell$ is real. This implies that the eigenvalues pair up as

$$
Q^{a}=\left(-Q^{j},-Q^{j-1} \ldots-Q^{1}, 0, Q^{1} \ldots Q^{j-1}, Q^{j}\right),
$$

where we assume that $N$ is odd, and $j=(N-1) / 2$. When $N$ is even the zero eigenvalue is dropped, and there are $j=N / 2$ pairs. Thus in general there are $j$ independent eigenvalues. For an arbitrary value of the loop, there is no simple relation between these eigenvalues.

Nevertheless, there are two exceptions. One is the perturbative QGP, where all $Q^{a}$ vanish. The other is the confined phase of a pure gauge theory,

$$
Q_{\text {conf }}^{a}=\pi T \frac{N+1-2 k}{N}, k=1, \ldots, N .
$$

That is, in the confined phase the eigenvalues are evenly distributed on the unit circle. The loops in the confined phase are

$$
\ell_{n}\left(Q_{\text {conf }}\right)= \begin{cases}(-1)^{j(N+1)} & , n=j N \\ 0 & , n \neq j N\end{cases}
$$


for general $N$. This behavior is easy to understand. Loops which carry $Z(N)$ charge vanish in the confined phase of the pure gauge theory, while those which are $Z(N)$ neutral do not.

For three colors,

$$
Q^{a}=(-Q, 0, Q)=2 \pi T(-q, 0, q) .
$$

The first Polyakov loop is then

$$
\ell=\frac{1}{3}(1+2 \cos (2 \pi q))
$$

In the confined phase of the pure gauge theory $q_{\mathrm{conf}}=1 / 3$. Similarly,

$$
l_{n}=\frac{1}{3}(1+2 \cos (2 \pi n q)) .
$$

In the presence of dynamical quarks there is no rigorous definition of a confined phase. Dynamical quarks act as a background $Z(N)$ field, so that any Polyakov loop is nonzero at nonzero temperature. Nevertheless, numerical simulations on the lattice find that $\ell$ is small, $\langle\ell\rangle \sim 0.1$, at the phase transition, at least for three colors and three light flavors. Thus we shall find it very convenient to compare results in the perturbative QGP to those in the confined phase of the pure gauge theory, as a limiting case of how large the effects can possibly be.

\subsection{Analytic continuation to Minkowski space-time}

Expanding around the background field in eq. (2.4), in Euclidean spacetime the energy of a quark becomes

$$
p_{0} \rightarrow p_{0}+Q^{a}
$$

while that of a gluon becomes

$$
p_{0} \rightarrow p_{0}+Q^{a b} ; \quad Q^{a b} \equiv Q^{a}-Q^{b},
$$

where $a$ and $b$ are color indices of the quark and the gluon in the double line basis [76]. Because of the usual boundary conditions in imaginary time, the energy $p_{0}$ for a fermion is an odd multiple of $\pi T$, while that for a boson is an even multiple of $\pi T$.

Although the momenta for fermions and bosons are rather different in Euclidean spacetime, it was argued previously that the proper procedure for analytic continuation to Minkowski spacetime is to continue the entire Euclidean energy to $-i E$, where $E$ is a continuous energy variable [76].

This has a simple but profound implication. In kinetic theory a given process is given by an integral over phase space of the square of a matrix elements times products of statistical distribution functions. Since the energies in Minkowski spacetime are as usual, then, for processes in which all the momenta are hard, the only change is in the $Q$-dependence of the statistical distribution functions. For processes involving soft momenta, it is also necessary to include the $Q$-dependence of the hard thermal loops as well [76]. We shall illustrate these general expectations by our explicit computations. It also suggests that it may be useful to treat the semi-QGP in kinetic theory, as for the perturbative QGP [100, 101]. 
The background gluon field acts as an imaginary chemical potential for colored particle, so that the statistical distribution functions for the quark, anti-quark, and gluon are, respectively,

$$
\begin{aligned}
\widetilde{n}_{a}(E) & =\frac{1}{e^{\left(E-i Q^{a}\right) / T}+1}, \quad \widetilde{n}_{\bar{a}}(E)=\frac{1}{e^{\left(E+i Q^{a}\right) / T}+1}, \\
n_{a b}(E) & =\frac{1}{e^{\left(E-i\left(Q^{a}-Q^{b}\right)\right) / T}-1} .
\end{aligned}
$$

Notice that the sign of $Q$ for the anti-quark, $+i Q^{a}$ in $\widetilde{n}_{\bar{a}}(E)$, is opposite to that for the quark, $-i Q^{a}$ in $\widetilde{n}_{a}(E)$. This is just like the change in sign for a quark chemical potential which is real. When the $Q^{a}=0$, of course these reduce to the usual Fermi-Dirac and Bose-Einstein distribution functions.

For future reference, it is useful to compute the statistical distribution functions, summed over all colors, in the confined phase of a pure gauge theory, eqs. (2.9) and (2.10). For the quark distribution function, this is

$$
\frac{1}{N} \sum_{a=1}^{N} \frac{1}{e^{\left(E-i Q_{\mathrm{conf}}^{a}\right) / T}+1}=\frac{1}{e^{N E / T}+1},
$$

while that of the gluon distribution function is

$$
\frac{1}{N^{2}} \sum_{a, b=1}^{N} \frac{1}{e^{\left(E-i Q_{\mathrm{conf}}^{a}+i Q_{\mathrm{conf}}^{b}\right) / T}-1}=\frac{1}{e^{N E / T}-1} .
$$

In the confined phase of the pure gauge theory, the only loops which contribute are those which wrap around a multiple of $N$ times. These can be considered as a type of "baryon", albeit in the pure gauge theory. Consequently, the energy which enters in the right hand side of eqs. (2.17) and (2.18) is not $E$, but $N$ times $E$. This rescaling of the energy will be seen to help explain the suppression of photon production at large $N$, eq. (4.81).

\subsection{Relation to lattice results}

In a matrix model all results are functions of the temperature, the coupling constant, and the background field $Q^{a}$. In this paper we take a conservative approach to fix the variation of the coupling constant, and the $Q^{a}$ 's, with temperature. We stress, however, that all of our results can easily be used to compute for alternate choices of $Q^{a}(T)$.

In order to give results as a function of temperature, in this work we determine the $Q^{a}$ 's from measurements of the (renormalized) Polyakov loop in numerical simulations on the lattice [85]. To do so, first it is necessary to remove perturbative corrections from the expectation value of the loop [88],

$$
\begin{aligned}
\ell(Q=0) & =1+\delta \ell(Q=0), \\
\delta \ell(Q=0) & =\frac{g^{2} C_{f} m_{D}}{8 \pi T}+\frac{g^{4} C_{f}}{(4 \pi)^{2}}\left[-\frac{N_{f}}{2} \ln 2+N\left(\ln \frac{m_{D}}{T}+\frac{1}{4}\right)\right]+\mathcal{O}\left(g^{5}\right),
\end{aligned}
$$



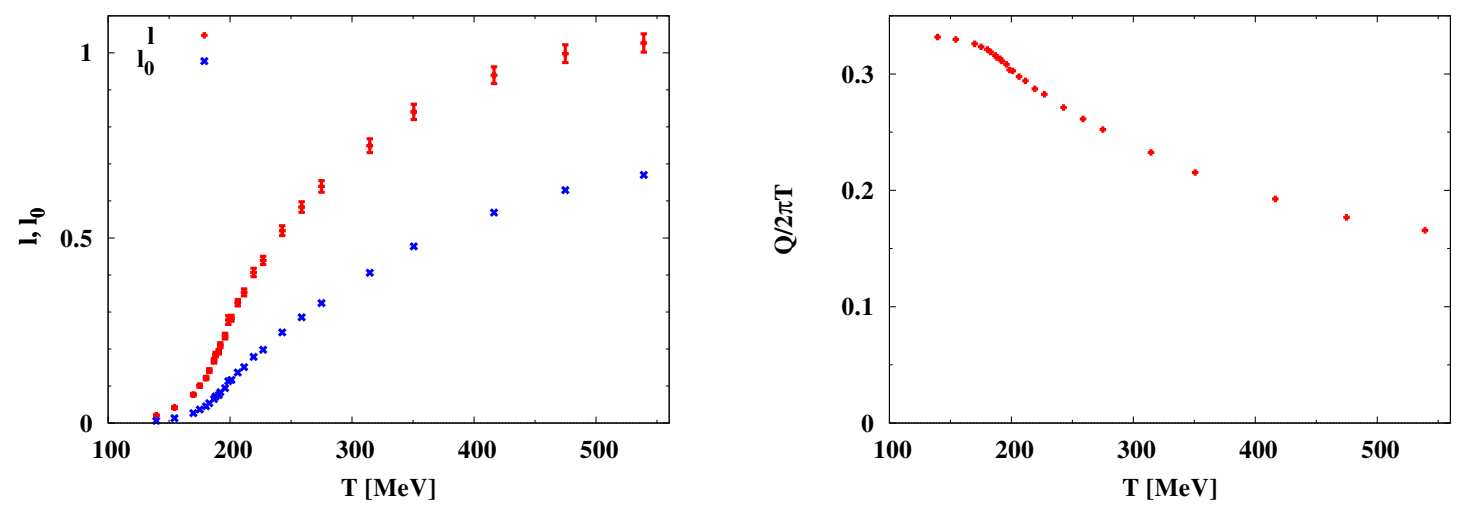

Figure 1. Left panel: the Polyakov loop $(l)$ determined from the lattice calculation [91], the Polyakov loop $\left(l_{0}\right)$ with the perturbative correction removed according to eq. (2.21) as a function of $T$. Right panel: $Q$ as a function of $T$. We set $\Lambda_{\overline{\mathrm{MS}}}=T_{c} / 1.35$, where $T_{c}=170 \mathrm{MeV}$.

where $C_{f} \equiv\left(N^{2}-1\right) /(2 N)$ is the Casimir for the fundamental representation, $m_{D}$ is the Debye mass of the gluon, and $N_{f}$ is number of quark flavors. We use the running coupling constant calculated in the modified minimal subtraction scheme at two-loop order, and the result for the Debye mass at one-loop order [89]:

$$
\begin{aligned}
g^{2}= & 24 \pi^{2}\left[\left(11 N-2 N_{f}\right)\left\{\ln \left(\frac{4 \pi T}{\Lambda_{\overline{\mathrm{MS}}}}\right)-\gamma_{E}\right\}+N_{f}(4 \ln 2-1)-\frac{11 N}{2}\right]^{-1}, \\
m_{D}^{2}= & \left(2 N+N_{f}\right) 4 \pi^{2} T^{2}\left[\left(11 N-2 N_{f}\right)\left\{\ln \left(\frac{4 \pi T}{\Lambda_{\overline{\mathrm{MS}}}}\right)-\gamma_{E}\right\}\right. \\
& \left.+4 N_{f} \ln 2-\frac{5 N^{2}+N_{f}^{2}+9 N_{f} /(2 N)}{2 N+N_{f}}\right]^{-1}
\end{aligned}
$$

where $\Lambda_{\overline{\mathrm{MS}}}$ is the renormalization mass scale in the modified minimal subtraction scheme, and $\gamma_{E} \simeq 0.57721$ is Euler's constant.

Equation (2.19) shows that a finite renormalization gives $\ell(Q=0)>1$. We assume that perturbative corrections exponentiate,

$$
\ell(Q)=e^{\delta \ell(Q=0)} \ell_{0}(Q) .
$$

Taking $\ell$ from numerical simulations of lattice QCD [91], and calculate $\ell_{0}$ from eq. (2.21), to obtain $Q$ from eq. (2.12). These quantities are plotted in figure 1 , by setting $\Lambda_{\overline{\mathrm{MS}}}=T_{c} / 1.35$. We see that $\ell_{0}$ is different from unity even around $\sim 3 T_{c}$, where $T_{c} \sim 170 \mathrm{MeV}$ is the pseudo-critical temperature of the phase transition [91].

There are several shortcomings of the above procedure. Instead of taking the value of the Polyakov loop from numerical simulations on the lattice, it would be better to have a complete effective model, whose parameters fit both thermodynamic quantities, such as the pressure, and the value of the Polyakov loop. With dynamical quarks, such a model does not, at present, exist, although it is actively under study. 
Indeed, the case of the pure gauge theory has been studied intensively in refs. [79, 80]. Fitting the parameters of the matrix model to the pressure, a good fit is found a computed quantity, the value of the 't Hooft loop. However, at a given temperature above $T_{c}$, the value of the Polyakov loop is uniformly larger than that measured on the lattice. Indeed, the width of the transition region in the matrix model, from $T_{c}$ to $\sim 1.2 T_{c}$, is much narrower than that indicated by the value of the (renormalized) Polyakov loop, from $T_{c}$ to $\sim 4.0 T_{c}[79,80]$.

In this vein, we note that two other effective models which predict the value of the Polyakov loop. Using either using the functional renormalization group [59-70], or background field methods [71-73], both models find a similarly narrow width for the region in which the Polyakov loop goes from zero to near unity. (We exclude Polyakov loop models, which by assumption fit the value of the Polyakov loop.)

Either all such models neglect important physics, or there is some subtle effect associated with the renormalization of the Polyakov loop (which is, after all, a nonlocal operator). At present we do not know the resolution of this problem. For this reason, we adopt the approach described above and simply take the value of the Polyakov loop from lattice simulations.

Another qualm about the prescription above is that in subtracting the perturbative corrections, we have used those computed in the ordinary perturbative vacuum, with $Q^{a}=0$. Clearly this should be done self-consistently, taking the correct $Q^{a} \neq 0$. Given the discrepancy between the lattice $Q^{a}$ and those in the matrix model, it seems prudent to delay such a technically challenging computation until this difference is better understood.

\section{Dilepton production rate}

\subsection{Computation to leading order}

We calculate the production rate of dileptons when $Q_{a} \neq 0$ in this subsection. To leading order in $\alpha_{\mathrm{em}}$, the production rate is

$$
\frac{d \Gamma}{d^{4} P}=-\frac{\alpha_{\mathrm{em}}}{24 \pi^{4} P^{2}} W_{\mu}^{\mu}(P)
$$

where $W_{\mu \nu}(P)$ is the Wightman correlator for two electromagnetic currents,

$$
W_{\mu \nu}(P)=\int d^{4} x e^{i P \cdot x}\left\langle j_{\nu}(0) j_{\mu}(x)\right\rangle,
$$

where $j^{\mu} \equiv e \sum_{f} \bar{\psi}_{f} \gamma^{\mu} \psi_{f}$, with $\psi$ is the quark operator with flavor index $f$. In thermal equilibrium, $W_{\mu \nu}$ is related to the imaginary part of the retarded photon self-energy as

$$
W_{\mu \nu}(P)=-2 n(E) \operatorname{Im} \Pi_{\mu \nu}^{R}(P),
$$

with

$$
\Pi_{\mu \nu}^{R}=-i \int d^{4} x e^{i P \cdot x} \theta\left(x^{0}\right)\left\langle\left[j_{\mu}(x), j_{\nu}(0)\right]\right\rangle .
$$

Here $P \equiv P_{1}+P_{2}$ with $P_{1}$ and $P_{2}$ being the momenta of the two leptons. 
At the leading order in the QCD coupling constant $g$, the contribution is obtained by $1 \rightarrow 2$ processes, illustrated in figure 2 . In this process, a quark anti-quark pair becomes a virtual photon, which then decays to a dilepton pair. This gives

$$
\begin{aligned}
\frac{d \Gamma}{d^{4} P}= & \frac{\alpha_{\mathrm{em}}}{24 \pi^{4} P^{2}} \sum_{f, \text { spin }} \int \frac{d^{3} k_{1}}{(2 \pi)^{3}} \frac{1}{2 E_{1}} \int \frac{d^{3} k_{2}}{(2 \pi)^{3}} \frac{1}{2 E_{2}}(2 \pi)^{4} \delta^{(4)}\left(P-K_{1}-K_{2}\right) \\
& \times|\mathcal{M}|^{2} \sum_{a=1}^{N} \widetilde{n}_{a}\left(E_{1}\right) \widetilde{n}_{\bar{a}}\left(E_{2}\right)
\end{aligned}
$$

where $f$ is a subscript for flavor running from 1 to $N_{f}$. We use the spacetime signature $(+---)$ in this paper; four-momenta are denoted by capital letters, $P^{\mu}=(E, \vec{p}), K_{1}^{\mu}=$ $\left(E_{1}, \vec{k}_{1}\right), K_{2}^{\mu}=\left(E_{2}, \vec{k}_{2}\right)$. The quark anti-quark pair is produced on it mass shell, $K_{1}^{2}=$ $K_{2}^{2}=0$, so $E_{1}=\left|\vec{k}_{1}\right| \equiv k_{1}, E_{2}=\left|\vec{k}_{2}\right| \equiv k_{2}$, and $P$ is time-like, $P^{2}>0$. Without loss of generality we can assume that the (virtual) photon energy is positive, $E>0$. Here $\widetilde{n}_{a}\left(E_{1}\right)$ and $\widetilde{n}_{\bar{a}}\left(E_{2}\right)$ are the statistical distribution functions for the quark and anti-quark in eq. (2.16). The square of matrix element is

$$
\sum_{\text {spin }}|\mathcal{M}|^{2}=8 e^{2} q_{f}^{2} K_{1} \cdot K_{2}=4 e^{2} q_{f}^{2} P^{2}
$$

where we have used $K_{1}^{2}=K_{2}^{2}=0$, and $q_{f}$ is the electromagnetic charge of the quark with flavor $f$ in the unit of $e$.

The result when $Q^{a}=0$ is well known [25]:

$$
\left.\frac{d \Gamma}{d^{4} P}\right|_{Q=0}=\frac{\alpha_{\mathrm{em}}^{2}}{12 \pi^{4}} \sum_{f} q_{f}^{2} N n(E) h(E, p) .
$$

For three flavors of quarks, $\sum_{f} q_{f}^{2}=2 / 3$. Here

$$
h(E, p) \equiv 1-\frac{2 T}{p} \ln \left(\frac{1+e^{-p_{-} / T}}{1+e^{-p_{+} / T}}\right),
$$

and

$$
p_{ \pm}=\frac{1}{2}(E \pm p)
$$

is the range of the quark momenta. Especially, when the dilepton pair is produced at rest, $\vec{p}=0$, the quark anti-quark pair are produced back to back, with $\vec{k}_{1}=-\vec{k}_{2}$. Their energies are equal, $E_{1}=E_{2}=E / 2$, and there is no integral over the quark momentum. The expression then reduces to

$$
\left.\frac{d \Gamma}{d^{4} P}\right|_{Q=0}=\frac{\alpha_{\mathrm{em}}^{2}}{12 \pi^{4}} \sum_{f} q_{f}^{2} N \widetilde{n}^{2}(E / 2) .
$$

This is natural, as the product of a Fermi-Dirac distribution function for the quark and anti-quark appears. 
Equation (3.5) illustrates our comment in section 2.3, that for hard momenta the only change when $Q^{a} \neq 0$ is in the change in the statistical distribution functions. To handle the $Q$-dependence of the $\widetilde{n}$ 's it is useful to note that

$$
\widetilde{n}_{a}\left(E_{1}\right) \widetilde{n}_{\bar{a}}\left(E_{2}\right)=n(E)\left(1-\widetilde{n}_{a}\left(E_{1}\right)-\widetilde{n}_{\bar{a}}\left(E_{2}\right)\right)
$$

remembering that $E=E_{1}+E_{2}$. This identity is familiar from when $Q^{a}=0$.

Using this, we can derive

$$
\operatorname{Im} \Pi_{\mu}^{R \mu}=\alpha_{\mathrm{e} m} \sum_{f} q_{f}^{2}\left(\frac{E^{2}-p^{2}}{p}\right) \sum_{a=1}^{N} \int_{p_{-}}^{p_{+}} d k\left(1-\widetilde{n}_{a}(k)-\widetilde{n}_{\bar{a}}(E-k)\right) .
$$

Here we wrote the photon retarded self-energy instead of the dilepton production rate for future convenience. We use energy-momentum conservation to write $E_{1}=k$ and $E_{2}=E-k$. This expression is useful when we compare to the results of ref. [99] at the end of this section, see eq. (3.23).

To leading order, we can write the rate for dilepton production when $Q^{a} \neq 0$ as a momentum dependent factor times that for $Q^{a}=0$,

$$
\left.\frac{d \Gamma}{d^{4} P}\right|_{Q \neq 0}=\left.f_{\overline{l l}}(Q) \frac{d \Gamma}{d^{4} P}\right|_{Q=0},
$$

where

$$
h(E, p) f_{\bar{l}}(Q)=\frac{1}{N} \sum_{a=1}^{N}\left(1-\frac{2 T}{p} \ln \left(\frac{1+e^{-\left(p_{-}-i Q^{a}\right) / T}}{1+e^{-\left(p_{+}-i Q^{a}\right) / T}}\right)\right) .
$$

This result can be evaluated by expanding in powers of $\exp \left(-\left(p_{\mp}-i Q^{a}\right) / T\right)$, and performing the sum over $a$ to obtain a series of Polyakov loops. For general $N$ all independent Polyakov loops, which run from $\ell_{1}$ to $\ell_{N-1}$, enter. The resulting expression is not very illuminating.

There are two cases in which one can obtain simple results. One is the confined phase of the pure gauge theory, eqs. (2.9) and (2.10). Then only loops which are a multiple of $N$ contribute, so that

$$
h(E, p) f_{l \bar{l}}\left(Q_{\text {conf }}\right)=1-\frac{2 T}{N p} \ln \left(\frac{1+e^{-N p_{-} / T}}{1+e^{-N p_{+} / T}}\right) .
$$

Another special case is three colors. Then one can rewrite $f_{\overline{l l}}$ so that only the first Polyakov loop appears,

$$
h(E, p) f_{l \bar{l}}(Q)=1-\frac{2 T}{3 p} \ln \left(\frac{1+3 \ell e^{-p_{-} / T}+3 \ell e^{-2 p_{-} / T}+e^{-3 p_{-} / T}}{1+3 \ell e^{-p_{+} / T}+3 \ell e^{-2 p_{+} / T}+e^{-3 p_{+} / T}}\right) .
$$

Of course $f_{l \bar{l}}(0)=1$ in the perturbative QGP, when $\ell=1$. In the confined phase where $\ell=0$, this agrees with the result in eq. (3.15).

In figure 3 we plot $f_{l \bar{l}}(Q)$ as a function of temperature for three colors. We do this for back to back dileptons, $p=0$, with $E=1 \mathrm{GeV}$. We see that the production of dileptons is 


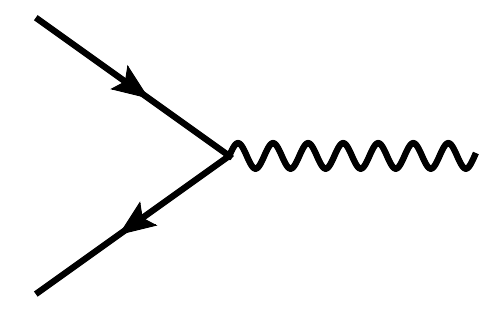

Figure 2. The 1 to 2 process which results the production of dilepton. The solid line denotes the quark while the wavy line denotes the (virtual) photon.

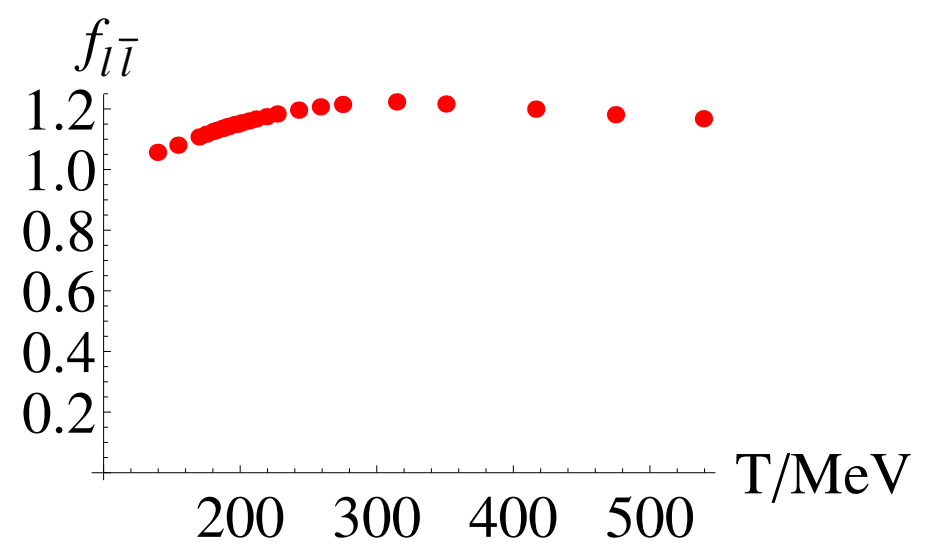

Figure 3. The ratio of dilepton production in the semi-QGP versus the perturbative QGP, $f_{l \bar{l}}$ in eq. (3.16), as a function of temperature. The dileptons are back to back, $p=0$, with a total energy $E=1 \mathrm{GeV}$.

not suppressed by the effect of the Polyakov loop, but moderately enhanced, by $\sim 20 \%$, at low temperatures $T \sim 300 \mathrm{MeV}$ in the semi-QGP.

This enhancement is rather unexpected. While the probability to produce either a single quark or anti-quark is small when the loop is small, that to produce a quark antiquark pair is greater in the semi-QGP than the perturbative QGP.

\subsection{Enhancement of dilepton production in the confined phase versus the perturbative Quark-Gluon Plasma}

To better understand the enhancement of dilepton production in the semi-QGP, relative to that in the perturbative QGP, we consider dilepton production for infinite $N$, comparing the confined phase to the perturbative QGP.

To simplify the analysis we consider dileptons which are produced back to back. This is most useful, because if the total spatial momentum of the pair vanishes, $p=0$, then each dilepton carries the same energy, $E / 2$, and we can ignore the integral over phase space as a common factor, independent of the $Q^{a}$. The effects of confinement, represented by $Q^{a} \neq 0$, can then be included just by computing the sum over the statistical distribution functions 
in eq. (3.5),

$$
\sum_{a=1}^{N} \widetilde{n}_{a}(E / 2) \widetilde{n}_{\bar{a}}(E / 2)=\sum_{a=1}^{N} \frac{1}{e^{\left(E / 2-i Q^{a}\right) / T}+1} \frac{1}{e^{\left(E / 2+i Q^{a}\right) / T}+1} .
$$

We note that, since the background field acts like a chemical potential for color, albeit imaginary, the sign for $Q^{a}$ is opposite between the quark and the anti-quark.

In the perturbative phase, $Q^{a}=0$, and eq. (3.17) is just $=N \widetilde{n}(E / 2)^{2}$, as appears in eq. (3.10).

In the semi-QGP, eq. (3.17) is computed by expanding each statistical distribution function in powers of $\exp \left(\left(E / 2 \mp i Q^{a}\right) / T\right)$,

$$
\sum_{a=1}^{N} \sum_{m=1}^{\infty} \sum_{m^{\prime}=1}^{\infty}(-)^{m+m^{\prime}} \exp \left(-\left(\left(m+m^{\prime}\right) E / 2+i\left(m-m^{\prime}\right) Q^{a}\right) / T\right) .
$$

This sum is especially easy to compute in the confined phase at infinite $N$. In that case, if $m \neq m^{\prime}$ the sum over $a$ gives $\sum \exp \left(i\left(m-m^{\prime}\right) Q^{a} / T\right)$; this is the Polyakov loop $\ell_{\left|m-m^{\prime}\right|}$, whose contribution vanishes at large $N$. The only nonzero contributions are from terms where $m=m^{\prime}$. For the terms in eq. (3.18) where $m=m^{\prime}$, though, the dependence on $Q^{a}$ drops out, cancelling identically between the quark and the anti-quark. The sums over $a$ and $m$ are then independent, and easy to do,

$$
\sum_{a=1}^{N} \sum_{m=1}^{\infty} e^{-m E / T}=\frac{N}{e^{E / T}-1}=N n(E)
$$

which does not vanish at large $N$. That is, while we start with only Fermi-Dirac distribution functions with $Q^{a} \neq 0$, in the confined phase at infinite $N$ we end up with a Bose-Einstein distribution function, which corresponds to the mesonic distribution function instead of the quark and the anti-quark. We also note that, previously we showed that the cancellation of the phases of the quark and the anti-quark are essential for the non-suppression of the dilepton rate at large $N$ by using the Boltzmann approximation [98]. From the discussion above, we see that the cancellation $\left(m=m^{\prime}\right)$ is important also in the case that we do not use the Boltzmann approximation.

This is a type of statistical confinement. Our simple model does not have true bound states, but there is a remnant of a bound state from the statistical sum over the $Q^{a}$ 's. It is this sum in eq. (3.18) which generates the Bose-Einstein distribution function in eq. (3.19).

Thus in the confined phase at infinite $N$,

$$
f_{\bar{l}}\left(Q_{\text {conf }}\right)_{N=\infty}=\frac{n(E)}{\widetilde{n}^{2}(E / 2)} .
$$

We note that this result also can be obtained by taking $p \rightarrow 0$ limit in eq. (3.14). This demonstrates a few interesting features. First, $f_{\overline{l l}}\left(Q_{\text {conf }}\right)_{N=\infty}$ is always larger than unity. Second, at low energy, the Bose-Einstein distribution function is enhanced, $n(E) \sim T / E$, while the Fermi-Dirac distribution function is constant, so

$$
f_{\bar{l}}\left(Q_{\text {conf }}\right)_{N=\infty}=\frac{4 T}{E}, \quad E \ll T .
$$


Thus under the given assumptions, at small energies dilepton production in the confined phase dominates that from the perturbative Quark-Gluon Plasma. This occurs because statistical confinement generates confined "bosons" from quark anti-quark pairs, and these confined bosons become over-occupied when their energies are much smaller than temperature. This occurs even though the probability to produce a single quark, or anti-quark, is strictly zero in the confined phase at infinite $N$. Nevertheless, we note that, when $E \leq g T$, we need to calculate with the HTL resummation [102] instead of our calculation, so our result eq. (3.21) can be altered in that energy region.

More generally, that the ratio of dilepton production in the confined phase to that in the perturbative QGP, $f_{l \bar{l}}(Q)$, is of order one, indicates that at all temperature dilepton production is of order $N$. This is one example of quark-hadron duality [103].

A similar enhancement of dilepton production in the confined phase was found previously by Lee, Wirstram, Zahed, and Hansson [14]. They considered a condensate for $A_{0}^{2}$. We can take our result in eq. (3.14), and expand up to quadratic order in the $Q^{a}$ 's, to obtain

$$
h(E, p) f_{\overline{l l}}(Q) \approx h(E, p)+\frac{1}{N p T} \sum_{a=1}^{N} Q_{a}^{2}\left(\widetilde{n}\left(p_{-}\right)\left(1-\widetilde{n}\left(p_{-}\right)\right)-\widetilde{n}\left(p_{+}\right)\left(1-\widetilde{n}\left(p_{+}\right)\right)\right),
$$

in agreement with eq. (7) of ref. [14]. These authors suggested that the enhancement of dilepton production in the confined phase may be related to the excess seen in heavy ion collisions for dilepton masses below that for the $\rho$-meson [24].

We can also make contact with results from Polyakov Nambu-Jona-Lasino (PNJL) models [56-58], especially with the computation of dilepton production by Islam, Majumder, Haque, and Mustafa [99]. To do so we need a simple identity. For three colors the sum of the Fermi-Dirac distribution function, with the $Q^{a}$ and $\ell$ as in eqs. (2.11) and (2.12), obeys

$$
\frac{1}{3} \sum_{a=1}^{3} \widetilde{n}_{a}(E)=\frac{1}{3} \sum_{a=1}^{3} \widetilde{n}_{\bar{a}}(E)=\frac{\ell \mathrm{e}^{-E / T}+2 \ell \mathrm{e}^{-2 E / T}+\mathrm{e}^{-3 E / T}}{1+3 \ell \mathrm{e}^{-E / T}+3 \ell \mathrm{e}^{-2 E / T}+\mathrm{e}^{-3 E / T}} .
$$

In the PNJL models of refs. [56-58, 99], when $\ell \neq 1$ the effective statistical distribution function is defined as the right hand side of eq. (3.23); e.g., eqs. (67) and (68) of ref. [57]. In refs. $[57,58,99]$ this effective distribution function was obtained by taking the derivative of the free energy, when $\ell \neq 1$, with respect to a given energy $E$. Since the free energy involves a sum over all colors, it is clear that defining the effective statistical distribution function in this way automatically gives a sum over all $\widetilde{n}_{a}(E)$ (or $\widetilde{n}_{\bar{a}}(E)$ ), which appears on the left hand side of eq. (3.23). (The identity of eq. (3.23) holds for the case of zero quark chemical potential. Then we can define $\ell$ to be real, and $\sum_{a} \widetilde{n}_{a}(E)$ and $\sum_{a} \widetilde{n}_{\bar{a}}(E)$ are equal. At nonzero quark chemical potential the loops in the fundamental and anti-fundamental representations are not equal, $\ell_{\mathbf{3}} \neq \ell_{\overline{\mathbf{3}}}$ [104]. In this instance, identities similar to eq. (3.23) hold for $\sum_{a} \widetilde{n}_{a}(E)$ and $\sum_{a} \widetilde{n}_{\bar{a}}(E)$ separately, and are again equal to those in the PNJL model, [57, 58, 99].)

In our matrix model the sum over the statistical distribution functions with all $Q^{a}$, $\sum_{a} \widetilde{n}_{a}(E)$ and $\sum_{a} \widetilde{n}_{\bar{a}}(E)$, enters naturally when we sum over all quark colors, eqs. (3.11) 
and (3.14). In the PNJL model calculation done in ref. [99], $\operatorname{Im} \Pi_{00}^{R}$ is given in eq. (4.36), and $\operatorname{Im} \Pi_{i i}^{R}$ by eq. (4.46). Taking the quarks to be massless, and using the fact that $\int_{p_{-}}^{p_{+}} d p \widetilde{n}_{\bar{a}}(E-p)=\int_{p_{-}}^{p_{+}} d p \widetilde{n}_{\bar{a}}(E)$, it can be shown that their result for $\operatorname{Im} \Pi_{\mu}^{R \mu}$ coincides identically with our eq. (3.12).

We emphasize that the equality between our results and the PNJL model [99] is valid only to leading order. In both cases, at leading order dilepton production is only a function of the Polyakov loop and the temperature. (As well as the quark mass and chemical potential, if one chooses to add them.) The results will certainly differ beyond leading order, and depend strongly upon the details of each effective model.

We note that, results for dilepton production at nonzero quark masses were computed in ref. [99] and by Satow and Weise [105]. There is a relatively mild dependence on the quark masses, apart from obvious kinematical constraints, such as the energy of the photon has to be greater than twice the quark mass.

\section{Photon production}

\subsection{Overview}

To leading order in $\alpha_{\mathrm{em}}$, the photon rate in the QGP is

$$
p \frac{d \Gamma_{\gamma}}{d^{3} p}=-\frac{1}{2(2 \pi)^{3}} g^{\mu \nu} W_{\mu \nu}(P) .
$$

Since a photon on its mass shell cannot decay directly to a quark anti-quark pair, this quantity vanishes at one-loop order.

In our model the first nonzero contribution occurs at two-loop order, from the diagrams shown in figure 4 . Cutting the diagrams we obtain $2 \rightarrow 2$ processes, which are Compton scattering and pair annihilation, both of order $e^{2} g^{2}$. We note that, consequently, and unlike the case of dilepton production to leading order, the results which we find have no direct correspondence in a PNJL model. One could compute photon production in a PNJL model, but since these models do not have dynamical gluons, the results will be very different from our matrix model. Each of these two processes has an infrared divergence when the momentum exchanged becomes soft [6]. The divergence is removed by using a resummed quark propagator for soft momenta, corresponding to the uncutted lower quark line in the left diagram of figure 4 , for example.

It was later realized that there exists another kinematic regime which contributes at the same order [9-11]. This corresponds to the case when the photon becomes collinear with quarks in the loop in the two diagrams of figure 4, more precisely, when the longitudinal momenta (defined with respect to photon momentum) of quarks remain hard, $\sim T$, and the transverse momenta are soft, $\sim g T$. Despite the reduced phase space, due to collinear enhancement, this regime was found to contribute equally as the $2 \rightarrow 2$ processes, in the analysis by Arnold, Moore and Yaffe (AMY) [10]: the collinear regime in this diagram also gives an overall $e^{2} g^{2}$ contribution to the photon emission rate. To clarify terminology, we will refer $2 \leftrightarrow 2$ rate as the contribution from figure 4 , excluding the rate in the collinear regime. We refer to the rest of the contributions $\sim e^{2} g^{2}$ as the collinear rate. 


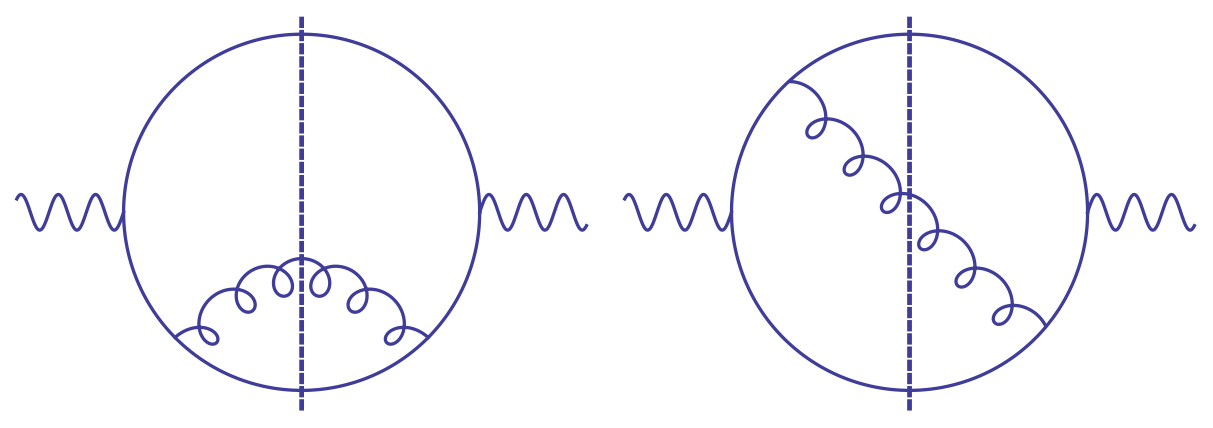

Figure 4. Two loops diagrams contributing to photon self-energy.

It turns out that the collinear rate goes beyond two-loop order: i.e. further additional soft gluons ladders in the collinear regime still contributes at the same order. Thus, the story is further complicated by interference among different diagrams. Physically, it is because that, the formation time of a photon, $t_{F} \sim 1 /\left(g^{2} T\right)$, is comparable to the mean free path for quarks, $\lambda \sim 1 /\left(g^{2} T\right)$. Since these two scales are similar, interference effects between scattering with multiple gluons must be included, which is the LandauPomeranchuk-Migdal (LPM) effect [10, 11]. Different diagrams add destructively, so that the LPM effect leads to additional suppression of collinear photon rate by $p^{-1 / 2}$ at large photon momentum $p \gg T$.

In this section we compute the production of real photons with large momentum in the presence of a nontrivial Polyakov loop. We begin by reviewing the computation of photon production to leading order in perturbation theory for $2 \rightarrow 2$ processes. We then generalize this to $Q^{a} \neq 0$. In contrast to dilepton production, we find that photon production is strongly suppressed in the confined phase. We give a simple explanation for this in terms of the initial state of the scattering.

We then give a detailed computation of the leading contributions to the collinear rate when $Q^{a} \neq 0$. In the presence of a nontrivial loop, the thermal mass of the quark is suppressed by a loop dependent factor, but it remains $\sim g \sqrt{N} T$ (here we explicitly wrote the $N$ dependence in the large $N$ limit). In contrast, the damping rate is suppressed by a factor of $1 / N$. Consequently, the mean free path of a quark or gluon is much larger, $\lambda \sim 1 /\left(g^{2} T\right)$, not $1 /\left(g^{2} N T\right)$ as in the $Q_{a}=0$ case. This implies that the LPM effect can be neglected at large $N$.

We compute the collinear processes when $Q^{a} \neq 0$ at large $N$. Doing so, we find that for three colors, the result is not that small, at least for physically reasonable values of the QCD coupling constant. Nevertheless, we find the result illuminating, to show how results can change in the semi-QGP.

\subsection{Hard momentum exchange with trivial Polyakov loop}

To establish notation on kinematics, we first review the computation of the differential photon rate for $2 \leftrightarrow 2$ processes at hard momentum exchange, in the case of $Q^{a}=0$ [6]. 
In kinetic theory, this is given by

$$
\begin{gathered}
p \frac{d \Gamma}{d^{3} p}=\sum_{i=1,2} \int \frac{d^{3} k_{1} d^{3} k_{2} d^{4} P^{\prime}}{(2 \pi)^{8} 8 E_{1} E_{2}} \delta^{(4)}\left(K_{1}+K_{2}-P-P^{\prime}\right) \delta\left(\left(P^{\prime}\right)^{2}\right) \theta\left(E^{\prime}\right) \\
\times n\left(E_{1}\right) n\left(E_{2}\right)\left(1 \pm n\left(E^{\prime}\right)\right)|\mathcal{M}|_{i}^{2}
\end{gathered}
$$

The summation $i$ represents the contribution of Compton scattering and pair annihilation, whose diagrams are shown in figure 5 . The statistical factors $n\left(E_{1}\right), n\left(E_{2}\right)$, and $n\left(E^{\prime}\right)$ can refer to either Fermi-Dirac or Bose-Einstein factors, depending upon the particular process. For Compton scattering, the statistical factor above is $1-\widetilde{n}\left(E^{\prime}\right)$, which corresponds to Pauli blocking; for pair annihilation, the corresponding factor $1+n\left(E^{\prime}\right)$, which represents Bose enhancement.

The incoming momenta are $K_{1}=\left(E_{1}, \vec{k}_{1}\right)$ and $K_{2}=\left(E_{2}, \vec{k}_{2}\right)$ the outgoing momentum $P^{\prime}=\left(E^{\prime}, \vec{p}^{\prime}\right)$, and $P=(E, \vec{p})$ is the photon momentum. We assume all particles are massless, so $E_{1}=\left|\vec{k}_{1}\right|$, etc. Whether the incoming or outgoing momenta are quarks or gluons depends upon the process considered. In this paper, we consider the case that the photon energy is much larger than temperature, $E \gg T$.

It is convenient to introduce the Mandelstam variables,

$$
\begin{aligned}
& s=\left(K_{1}+K_{2}\right)^{2}, \\
& t=\left(K_{1}-P\right)^{2}, \\
& u=\left(K_{2}-P\right)^{2} .
\end{aligned}
$$

With our kinematics,

$$
s \geq 0 ; t, u \leq 0 .
$$

We decompose the incoming momenta $\vec{k}_{1}$ and $\vec{k}_{2}$ into components parallel and perpendicular to the photon momentum $\vec{p}$, with

$$
\begin{aligned}
& k_{1}^{\|}=\frac{t}{2 p}+E_{1}, \quad\left(k_{1}^{\perp}\right)^{2}=-\frac{t^{2}}{4 p^{2}}-\frac{t E_{1}}{p}, \\
& k_{2}^{\|}=\frac{u}{2 p}+E_{2}, \quad\left(k_{2}^{\perp}\right)^{2}=-\frac{u^{2}}{4 p^{2}}-\frac{u E_{2}}{p},
\end{aligned}
$$

and where ${\overrightarrow{k_{1}}}^{\perp} \cdot{\overrightarrow{k_{2}}}^{\perp}=k_{1}^{\perp} k_{2}^{\perp} \cos \left(\phi_{1}-\phi_{2}\right)$. We can then convert the variables of integration as

$$
d^{3} k_{1} d^{3} k_{2}=\frac{1}{4} d \phi_{1} d \phi_{2} d k_{1}^{\|} d k_{2}^{\|} d\left(k_{1}^{\perp}\right)^{2} d\left(k_{2}^{\perp}\right)^{2}=\frac{1}{4} d \phi_{1} d \phi_{2} \frac{E_{1} E_{2}}{p^{2}} d t d u d E_{1} d E_{2} .
$$

The integrand only depends on $\phi \equiv \phi_{1}-\phi_{2}$ through $\delta\left(\left(P^{\prime}\right)^{2}\right)$ :

$$
\delta\left(P^{\prime 2}\right)=\delta\left(2 E_{1} E_{2}-2 k_{1}^{\|} k_{2}^{\|}-2 k_{1}^{\perp} k_{2}^{\perp} \cos \phi-s\right) .
$$

The angular integrals are easily done to give the following result:

$$
\int \frac{d^{3} k_{1} d^{3} k_{2}}{8 E_{1} E_{2}} \delta\left(\left(P^{\prime}\right)^{2}\right)=\int \frac{d t d u d x d y(2 \pi)}{32 p} \frac{1}{\sqrt{a y^{2}+b y+c}},
$$


where we define

$$
x=E_{1}+E_{2} \quad ; \quad y=E_{1}-E_{2},
$$

and

$$
\begin{aligned}
a & =-\frac{s^{2}}{4} \\
b & =\left(\frac{x}{2}-p\right)\left(t^{2}-u^{2}\right), \\
c & =-\frac{1}{4}(t-u)^{2} x^{2}+p s^{2} x-p^{2} s^{2}-u t s .
\end{aligned}
$$

We start with the integral over $y$. Let $y_{ \pm}$be the solutions of the quadratic form in $y$, $a y_{ \pm}^{2}+b y_{ \pm}+c=0$. The integral over $y$ runs from $y_{-}$to $y_{+}$, where $a y^{2}+b y+c \geq 0$.

In considering the quadratic form in $y$, we assumed that $b^{2}-4 a c \geq 0$. A bit of algebra shows that this determines the range for $x$ to be $x \geq p+s /(4 p)$. As the energy $E^{\prime}=E_{1}+E_{2}-p=x-p$, we automatically satisfy the condition that this particle has positive energy, $E^{\prime}>0$, and can set $\theta\left(E^{\prime}\right)=1$ in eq. (4.2).

Since we assume that the incoming momenta are hard, the distribution functions, $n\left(E_{1}\right)$ and $n\left(E_{2}\right)$, can be replaced by their Boltzmann forms, $\exp \left(-E_{1} / T\right)$ and $\exp \left(-E_{2} / T\right)$. Consequently, the product of statistical distribution functions in eq. (4.2) reduces to

$$
n\left(E_{1}\right) n\left(E_{2}\right)\left(1 \pm n\left(E^{\prime}\right)\right) \sim e^{-\left(E_{1}+E_{2}\right) / T}\left(1 \pm \frac{1}{e^{E^{\prime} / T} \mp 1}\right)=e^{-x / T}\left(1 \pm \frac{1}{e^{(x-p) / T} \mp 1}\right) .
$$

This vastly simplifies the integral over phase space. In general, the product in eq. (4.11) is a function of both sum and difference of the energies, $x$ and $y$. For hard momenta, though, this reduces just to a function of the sum, of $x$. In appendix A, we show that corrections to eq. (4.11) are in fact exponentially suppressed, as one would expect.

This allows us to immediately perform the integral over $y$. Although the coefficients $b$ and $c$, and $y_{ \pm}$, are all functions of $x$, in the end we obtain simply

$$
\int_{y_{-}}^{y_{+}} \frac{d y}{\sqrt{a y^{2}+b y+c}}=\left.\frac{1}{\sqrt{-a}} \sin ^{-1}\left(\frac{2 a y+b}{\sqrt{b^{2}-4 a c}}\right)\right|_{y_{-}} ^{y_{+}}=\frac{\pi}{\sqrt{-a}} .
$$

We can then readily evaluate the integral over $x$,

$$
\int_{p+\frac{s}{4 p}}^{\infty} d x e^{-x / T}\left(1 \pm \frac{1}{e^{(x-p) / T} \mp 1}\right)=\mp T e^{-p / T} \ln \left(1 \mp e^{-s /(4 p T)}\right) .
$$

Therefore, the phase space integrals give

$$
\begin{aligned}
& \int \frac{d^{3} k_{1} d^{3} k_{2}}{8 E_{1} E_{2}} \delta\left(\left(P^{\prime}\right)^{2}\right) n\left(E_{1}\right) n\left(E_{2}\right)\left(1 \pm n\left(E^{\prime}\right)\right) \\
& =\int d t d u \frac{\pi^{2}}{8 p s}\left(\mp T e^{-p / T}\right) \ln \left(1 \mp e^{-s /(4 p T)}\right) .
\end{aligned}
$$

To proceed, we consider Compton scattering and pair annihilation separately, since it involves a calculation of the matrix element squared. For Compton scattering off of quarks 
and antiquarks, the squared amplitude is given by

$$
|\mathcal{M}|^{2}=2 \sum_{f} q_{f}^{2}(4 \pi)^{2} \alpha_{\mathrm{em}} \alpha_{s} \frac{N^{2}-1}{2}(-8)\left(\frac{s}{t}+\frac{t}{s}\right) .
$$

The first term in eq. (4.15), $\sim s / t$, is logarithmically divergent when integrated over $t$. The second term, $\sim t / s$, does not produce a logarithmic divergence. As we show below, it is the logarithmic divergence that gives rise to leading logarithmic results in photon production, and we can ignore the second term.

Remembering that $t$ is negative, the logarithmic divergence happens for small $-t$, and invalidates the kinetic theory description. The standard treatment is to introduce an IR cutoff $\mu$ for the spatial component of the exchanged momentum, $\left|\vec{k}_{1}-\vec{p}\right|>\mu$. We assume that this cutoff lies between the hard and soft scales in the problem, $\mu \gg g T$ and $\mu \ll T$. Near zero, the integral over $t$ is modified as follows:

$$
\left|\vec{k}_{1}-\vec{p}\right|>\mu \Rightarrow t+\mu^{2} \leq\left(E_{1}-p\right)^{2} .
$$

On the other hand, the integration range of $y$ is given by $a y^{2}+b y+c \geq 0$, which as $t \rightarrow 0$ takes the following form

$$
2\left|E_{1}-p\right|=|x+y-2 p| \leq 2 \sqrt{\frac{t}{s}\left(4 p^{2}+s-4 p x\right)} .
$$

Comparing eqs. (4.16) and (4.17) and noting $x>p$, the lower cutoff on $-t$ is

$$
-t \geq \frac{s}{4 p(x-p)} \mu^{2} .
$$

Since we compute only to leading logarithmic accuracy, in the integral over $-t$ we can simply take the lower limit to be $\mu^{2}$, to obtain

$$
\int_{\mu^{2}}^{s} d(-t) \frac{s}{t}=-s \ln \left(\frac{s}{\mu^{2}}\right) .
$$

This leaves an integral over $u$. However, since $s=-t-u$, we can trade this for an integral over $s$. The final $s$-integral becomes

$$
\begin{aligned}
& \int_{\mu^{2}}^{\infty} \frac{d s}{s} \ln \left(1+e^{-s /(4 p T)}\right)(-s) \ln \left(\frac{s}{\mu^{2}}\right) \\
& \sim-\ln \left(\frac{p T}{\mu^{2}}\right) \int_{0}^{\infty} d s \ln \left(1+e^{-s /(4 p T)}\right)=-\frac{\pi^{2}}{12}(4 p T) \ln \left(\frac{p T}{\mu^{2}}\right),
\end{aligned}
$$

where we have replaced $\ln \left(s / \mu^{2}\right)$ by $\ln \left(p T / \mu^{2}\right)$ and extend the lower bound of the integration to zero. This is justified as to leading logarithmic order the region of integration is $s \sim p T \gg \mu^{2}$.

Collecting everything together, we obtain

$$
p \frac{d \Gamma}{d^{3} p} \simeq \sum_{f} q_{f}^{2} \frac{\alpha_{\mathrm{em}} \alpha_{s}}{48 \pi^{2}}\left(N^{2}-1\right) T^{2} e^{-p / T} \ln \left(\frac{p T}{\mu^{2}}\right) .
$$



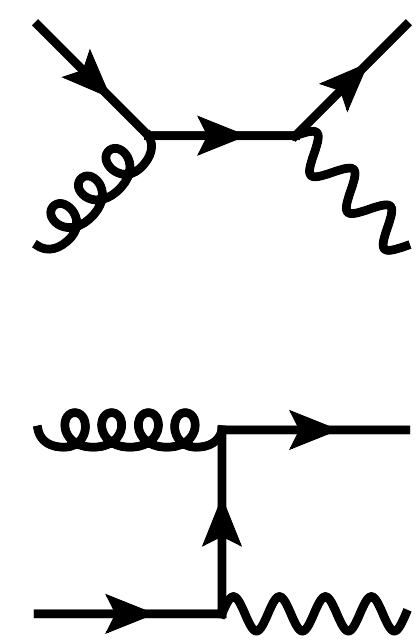
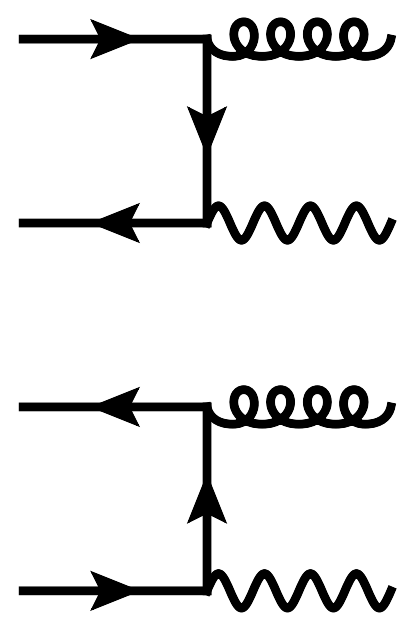

Figure 5. The diagrams for the Compton scattering (left) and the pair annihilation (right). The solid line corresponds to a quark, the wavy line to the photon, and the curly line to the gluon, respectively. The Compton scattering includes $s$ and $t$ channel processes while pair annihilation includes the $t$ and $u$ channel processes.

The case of annihilation proceeds similarly. The squared amplitude is given by

$$
|\mathcal{M}|^{2}=\sum_{f} q_{f}^{2}(4 \pi)^{2} \alpha_{\mathrm{em}} \alpha_{s} \frac{N^{2}-1}{2} 8\left(\frac{u}{t}+\frac{t}{u}\right) .
$$

Since the integrand is symmetric in $t$ and $u$, both $t$ and $u$-channels contribute the same to leading logarithmic order. The integral in the $t$-channel becomes

$$
\int_{s}^{\mu^{2}} d(-t) \frac{u}{t}=\mu^{2}-s+s \ln \frac{s}{\mu^{2}} .
$$

We again keep only the logarithm and use the same trick as in eq. (4.20) to obtain the leading logarithmic result. Note that there is Bose-Einstein enhancement for the annihilation process:

$$
\begin{aligned}
& \int_{\mu^{2}}^{\infty} \frac{d s}{s} \ln \left(1-e^{-s /(4 p T)}\right) s \ln \left(\frac{s}{\mu^{2}}\right) \\
& \sim \ln \left(\frac{p T}{\mu^{2}}\right) \int_{0}^{\infty} d s \ln \left(1-e^{-s /(4 p T)}\right)=-\frac{\pi^{2}}{6}(4 p T) \ln \left(\frac{p T}{\mu^{2}}\right) .
\end{aligned}
$$

The $u$-channel gives an identical contribution.

Collecting everything together, the combination of Compton scattering in the $t$ channel, and pair annihilation in the $t$ and $u$ channels, is

$$
p \frac{d \Gamma}{d^{3} p} \simeq \sum_{f} q_{f}^{2} \frac{\alpha_{\mathrm{em}} \alpha_{s}}{16 \pi^{2}}\left(N^{2}-1\right) T^{2} e^{-p / T} \ln \left(\frac{p T}{\mu^{2}}\right)
$$



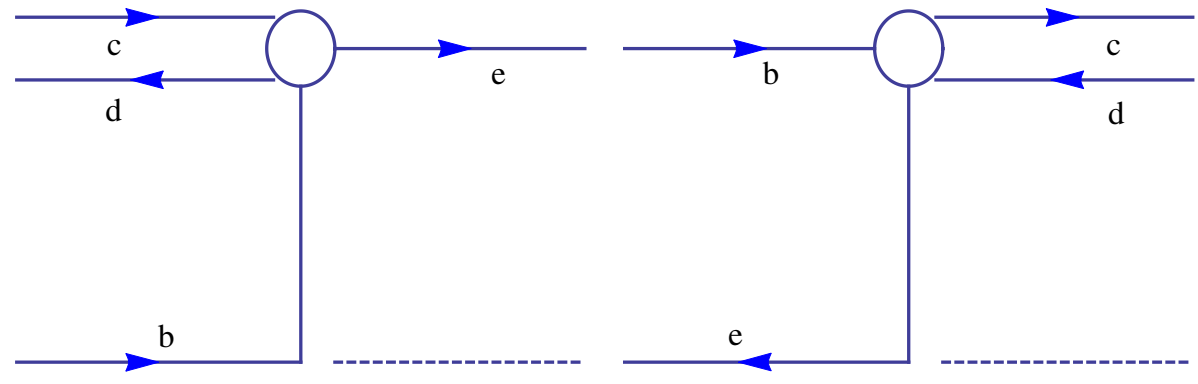

Figure 6. The color labeling of Compton scattering (left) and pair annihilation (right). The double line corresponds to a gluon, a single line to a quark or antiquark. The color flow does not involve color neutral photon, which we still indicate with a dashed line. The quark-gluon vertices are drawn as an empty circle. They have their own graphic representation [76], but this is not needed here. The Feynman diagrams obtained by crossing symmetry are identical in the flow of colors.

\subsection{Hard momentum exchange with nontrivial Polyakov loop}

In the previous section, we computed the matrix elements for the diagrams which contribute to photon production at leading logarithmic order. Once we work in terms of Minkowski variables, there is no change in computing in the presence of a background field for the Polyakov loop.

The only change in a background field arises from the modification of the statistical distribution functions. We start with the case of Compton scattering, as illustrated in the figure in the left hand side of figure 6 . In this case, the incoming momenta are those of a gluon, with momentum $K_{1}$, and a quark, with momentum $K_{2}$. Consequently, in the statistical distribution functions we replace the gluon energy as $E_{1} \rightarrow E_{1}+i\left(Q^{c}-Q^{d}\right)$, while the quark energy $E_{2} \rightarrow E_{2}+i Q^{b}$. Similarly, the energy of the outgoing quark becomes $E^{\prime} \rightarrow E^{\prime}+i Q^{e}$.

With the color labeling in figure 6 , the thermal distribution functions when $Q^{a} \neq 0$ are

$$
\begin{aligned}
\int_{p+s /(4 p)}^{\infty} d x e^{-\left(x+i\left(Q_{b}+Q_{c}-Q_{d}\right)\right) / T}\left(1-\frac{1}{e^{\left(x-p+i Q_{e}\right) / T}+1}\right) \\
=e^{-i\left(Q_{b}+Q_{c}-Q_{d}\right) / T} \int_{p+s / 4 p}^{\infty} d x \sum_{n=0}^{\infty} e^{-x / T}(-1)^{n} e^{-n\left(x-p+i Q_{e}\right) / T} \\
=e^{-i\left(Q_{b}+Q_{c}-Q_{d}\right) / T} \sum_{n=1}^{\infty} \frac{(-1)^{n+1}}{n} T e^{-p / T} e^{-n s /(4 p T)} e^{-i(n-1) Q_{e} / T}
\end{aligned}
$$

To obtain the leading logarithmic result, we recall eq. (4.20): eq. (4.26) should be integrated over $s$. The $s$-dependent factor gives rise to an additional factor of $1 / n$ :

$$
\int_{0}^{\infty} d s \exp \left(-\frac{n}{4 p T} s\right)=\frac{4 p T}{n} .
$$

It is sufficient to calculate the ratio of photon rate with $Q^{a} \neq 0$ to that in the perturbative limit, $Q^{a}=0$. We will thus only keep track of $Q$-dependent factor $\sum_{n}(-1)^{n+1} \exp \left(-i\left(Q_{b}+\right.\right.$ $\left.\left.\left.Q_{c}-Q_{d}+(n-1) Q_{e}\right)\right) / T\right) / n^{2}$. To proceed, we then need the form of the quark-gluon vertex 
in the double line notation [76], appearing in the matrix element squared, eq. (2.3). We then multiply eq. (4.26) by the product of two quark-gluon vertices,

$$
\begin{aligned}
& \sum_{b, c, d, e=1}^{N}\left(T^{d c}\right)_{b e}\left(T^{c d}\right)_{e b} \sum_{n=1}^{\infty} \frac{(-1)^{n+1}}{n^{2}} e^{-i\left(Q_{b}+Q_{c}-Q_{d}+(n-1) Q_{e}\right) / T} \\
= & \sum_{b, c, d, e=1}^{N} \frac{1}{2}\left(\delta_{b d} \delta_{c e}-\frac{2}{N} \delta_{b d} \delta_{c e} \delta_{c d} \delta_{b e}+\frac{1}{N^{2}} \delta_{c d} \delta_{b e}\right) \sum_{n=1}^{\infty} \frac{(-1)^{n+1}}{n^{2}} e^{-i\left(Q_{b}+Q_{c}-Q_{d}+(n-1) Q_{e}\right) / T} \\
= & \left(\frac{N^{2}-1}{2 N}\right) \sum_{n=1}^{\infty} \frac{(-1)^{n+1}}{n^{2}} \operatorname{tr} \mathbf{L}^{n} .
\end{aligned}
$$

When all $Q^{\prime} s$ are zero, this reduces to

$$
\left(\frac{N^{2}-1}{2}\right) \sum_{n=1}^{\infty} \frac{(-1)^{n+1}}{n^{2}}=\left(\frac{N^{2}-1}{2}\right) \frac{\pi^{2}}{12} .
$$

For Compton scattering, the ratio of this contribution when $Q^{a} \neq 0$, to that for $Q^{a}=0$, is just the ratio of eqs. (4.28) and (4.29),

$$
f_{\text {Comp }}(Q)=\frac{12}{\pi^{2}} \sum_{n=1}^{\infty} \frac{(-1)^{n+1}}{n^{2}} \ell_{n},
$$

where $\ell_{n}$ is the $n$-th Polyakov loop in eq. (2.7).

The case of annihilation is similar. In the presence of a background color charge, the thermal distribution becomes

$$
\begin{aligned}
& \int_{p+s /(4 p)}^{\infty} d x e^{-\left(x+i Q_{b}-i Q_{e}\right) / T}\left(1+\frac{1}{e^{\left(x-p+i Q_{c}-i Q_{d}\right) / T}-1}\right) \\
& =\sum_{n=1}^{\infty} \frac{1}{n} T e^{-p / T} e^{-n s /(4 p T)} e^{-i\left(Q_{b}-Q_{e}+(n-1)\left(Q_{c}-Q_{d}\right) / T\right.}
\end{aligned}
$$

Again, the integration of $e^{-n s /(4 p T)}$ over $s$ picks up an additional factor of $1 / n$. The color sum for scattering in the $t$-channel becomes

$$
\begin{aligned}
& \sum_{b, c, d, e=1}^{N}\left(T^{c d}\right)_{b e}\left(T^{d c}\right)_{e b} \sum_{n=1}^{\infty} \frac{1}{n^{2}} e^{-i\left(Q_{b}-Q_{e}+(n-1)\left(Q_{c}-Q_{d}\right)\right) / T} \\
= & \frac{1}{2} \sum_{b, c, d, e=1}^{N}\left(\delta_{b c} \delta_{d e}-\frac{2}{N} \delta_{b c} \delta_{d e} \delta_{c d} \delta_{b e}+\frac{1}{N^{2}} \delta_{c d} \delta_{b e}\right) \sum_{n=1}^{\infty} \frac{1}{n^{2}} e^{-i\left(Q_{b}-Q_{e}+(n-1)\left(Q_{c}-Q_{d}\right)\right) / T} \\
= & \frac{1}{2} \sum_{n=1}^{\infty} \frac{1}{n^{2}}\left(\left(\operatorname{tr} \mathbf{L}^{n}\right)^{2}-1\right)
\end{aligned}
$$

When all $Q^{\prime} s$ are zero, eq. (4.32) becomes

$$
\left(\frac{N^{2}-1}{2}\right) \sum_{n=1}^{\infty} \frac{1}{n^{2}}=\left(\frac{N^{2}-1}{2}\right) \frac{\pi^{2}}{6} .
$$


Scattering in the $u$-channel gives a result identical to that in the $t$-channel. Therefore, the suppression factor for annihilation is given by

$$
f_{\text {pair }}(Q)=\frac{1}{N^{2}-1} \frac{6}{\pi^{2}} \sum_{n=1}^{\infty} \frac{1}{n^{2}}\left(N^{2} \ell_{n}^{2}-1\right) .
$$

Remember that Compton scattering is $1 / 3$ of the total for $2 \rightarrow 2$ scatterings, eqs. (4.21) and (4.25). Summing over Compton scattering and pair annihilation, to leading logarithmic order, we obtain the contribution from $2 \rightarrow 2$ scattering from hard momenta in the semi-QGP,

$$
\begin{aligned}
p \frac{d \Gamma}{d^{3} p} & =\sum_{f} q_{f}^{2} \frac{1}{16}\left(N^{2}-1\right) \alpha_{\mathrm{em}} \alpha_{s} \frac{T^{2}}{\pi^{2}} e^{-p / T} \ln \left(\frac{p T}{\mu^{2}}\right) f_{\gamma}(Q), \\
f_{\gamma}(Q) & =\frac{1}{3}\left(f_{\text {Comp }}(q)+2 f_{\text {pair }}(q)\right) .
\end{aligned}
$$

These expressions can be more simply expressed when $N=3$ in terms of $Q^{a}=2 \pi T(-q, 0, q)$, eq. (2.13):

$$
\begin{aligned}
f_{\text {Comp }}(q) & =1-8 q^{2}, \\
f_{\text {pair }}(q) & =1-6 q+9 q^{2}, \\
f_{\gamma}(Q) & =1-4 q+\frac{10}{3} q^{2} .
\end{aligned}
$$

The results for more than three colors are similar, simple quadratic polynomials in the $Q^{a}$ 's. That for $f_{\text {Comp }}(Q)$ involves the $Q^{a}$, while that for $f_{\text {pair }}(Q)$ is a function of the differences, $Q^{a}-Q^{b}$.

We also note that exactly the same functions of $q^{a}$ enter into collisional energy loss for a heavy quark in the semi-QGP. Because of the historical convention, the function for Compton scattering in photon production, $f_{\text {Comp }}(q)$, is identical to that for Coulomb scattering of a heavy quark, eq. (33) of ref. [85]. Similarly, the function for pair annihilation in photon production, $f_{\text {pair }}(Q)$, is the same function as for Compton scattering of a heavy quark, eq. (45) of ref. [85]. While these two functions are the same, in detail they enter differently into collisional energy loss for a heavy quark, times different logarithms of the energy.

\subsection{Soft momentum exchange}

We now compute the contribution to photon production when the momentum exchanged is soft. This case is simpler than when the momentum exchanged is hard, and so we treat the case of a nontrivial Polyakov loop at the outset.

We follow the analysis of Baier, Nakkagawa, Niegawa, and Redlich [6]. We begin the computation in imaginary time, and then analytically continue the external momentum. The photon self-energy in the imaginary time is

$$
\Pi_{\mu}^{\mu}(P)=2 e^{2} \sum_{f} q_{f}^{2} \sum_{a=1}^{N} T \sum_{k^{0}} \int \frac{d^{3} k}{(2 \pi)^{3}} \operatorname{tr}\left[\gamma^{\mu} S_{a}^{*}(K) \gamma_{\mu} S_{a}(K-P)\right] .
$$


The overall factor of two arises because $K$ or $K-P$ can be a soft momentum: we have chosen only $K$ to be soft. Thus the momenta $K-P$ is hard, so we can use the bare quark propagator, $S_{a}(K-P)$. For the quark with soft momenta it is necessary to use a propagator, $S_{a}^{*}(K)$, which is resummed with Hard Thermal Loops (HTLs) in the presence of $Q^{a} \neq 0[76]$,

$$
\begin{aligned}
S_{a}(K) & =\int_{-\infty}^{\infty} \frac{d \omega}{2 \pi} \frac{\rho(\omega, \vec{k})}{\omega-i \tilde{k}_{0}}, \\
S_{a}^{*}(K) & =\int_{-\infty}^{\infty} \frac{d \omega}{2 \pi} \frac{\rho_{a}^{*}(\omega, \vec{k})}{\omega-i \tilde{k}_{0}}
\end{aligned}
$$

with $\tilde{k}_{0} \equiv k_{0}+Q_{a}$ and the quark spectral functions are

$$
\begin{aligned}
\rho(\omega, \vec{k}) & =2 \pi \varepsilon(\omega) \not K \delta\left(K^{2}\right), \\
\rho_{a}^{*}(\omega, \vec{k}) & =\frac{\gamma^{0}-\vec{\gamma} \cdot \hat{k}}{2} \rho_{a+}^{*}(\omega, \vec{k})+\frac{\gamma^{0}+\vec{\gamma} \cdot \hat{k}}{2} \rho_{a-}^{*}(\omega, \vec{k}),
\end{aligned}
$$

where $\varepsilon(\omega)$ is the sign function. We note that the bare quark spectral function $\rho(\omega, \vec{k})$ does not have its color index. The HTL spectral functions are a sum of pole and cut terms,

$$
\begin{aligned}
\rho_{a \pm}^{*}(\omega, \vec{k})= & 2 \pi\left[Z_{ \pm a}(k) \delta\left(\omega-\omega_{ \pm a}(k)\right)+Z_{\mp a}(k) \delta\left(\omega+\omega_{\mp a}(k)\right)\right] \\
& +\theta\left(k^{2}-\omega^{2}\right) \rho_{a \pm}^{\text {spacelike }}(\omega, \vec{k}) .
\end{aligned}
$$

The quark quasi-particles have a thermal mass $m_{\mathrm{qk} a}$, a dispersion relation $\omega_{ \pm a}(k)$, and residue $Z_{ \pm a}(k)(k=|\vec{k}|)$. Explicitly,

$$
\begin{aligned}
Z_{ \pm a}(k) & =\frac{\omega_{ \pm a}^{2}(k)-k^{2}}{2 m_{\mathrm{qk}^{a}}^{2}} \\
\omega_{ \pm a}(k) \mp k & =\frac{m_{\mathrm{qk}}^{2} a}{k}\left[\left(1 \mp \frac{\omega_{ \pm a}(k)}{k}\right) Q_{0}\left(\frac{\omega_{ \pm a}(k)}{k}\right) \pm 1\right],
\end{aligned}
$$

where

$$
Q_{0}(x) \equiv \frac{1}{2} \ln \left(\frac{x+1}{x-1}\right) .
$$

The explicit form of the cut term from Landau damping, $\rho_{a \pm}^{\text {spacelike }}$, is irrelevant for our analysis. The result for the quark quasi-particle mass $m_{\mathrm{qk} a}$ is given later.

Introducing a spectral representation for the propagators,

$$
\begin{aligned}
\Pi_{\mu}^{\mu}(P)=-2 e^{2} \sum_{f} q_{f}^{2} \sum_{a=1}^{N} & \int \frac{d^{3} k}{(2 \pi)^{3}} \int \frac{d \omega_{1}}{2 \pi} \int \frac{d \omega_{2}}{2 \pi} \\
\times & \frac{\left(\widetilde{n}_{a}\left(\omega_{2}\right)-\widetilde{n}_{a}\left(\omega_{1}\right)\right)}{-i p^{0}+\omega_{1}-\omega_{2}} \operatorname{tr}\left[\gamma^{\mu} \rho_{a}^{*}\left(\omega_{1}, \vec{k}\right) \gamma_{\mu} \rho\left(\omega_{2}, \vec{k}-\vec{p}\right)\right],
\end{aligned}
$$

Since for massless quarks their spectral density has only a vector component,

$$
\operatorname{tr}\left[\gamma^{\mu} \rho_{a}^{*}\left(\omega_{1}, \vec{k}\right) \gamma_{\mu} \rho\left(\omega_{2}, \vec{k}-\vec{p}\right)\right]=-2 \operatorname{tr}\left[\rho_{a}^{*}\left(\omega_{1}, \vec{k}\right) \rho\left(\omega_{2}, \vec{k}-\vec{p}\right)\right] .
$$


Now we compute the discontinuity in the amplitude, as we analytically continue the photon energy $p_{0} \rightarrow-i E \pm \epsilon$, for infinitesimal $\epsilon$,

$$
\begin{aligned}
\operatorname{Disc} \Pi_{\mu}^{\mu}(P)=+2 e^{2} \sum_{f} q_{f}^{2} \sum_{a=1}^{N} \int \frac{d^{3} k}{(2 \pi)^{3}} \int \frac{d \omega}{2 \pi} & \left(\widetilde{n}_{a}(\omega-E)-\widetilde{n}_{a}(\omega)\right) \\
& \times \operatorname{tr}\left[\rho_{a}^{*}(\omega, \vec{k}) \rho(\omega-E, \vec{k}-\vec{p})\right],
\end{aligned}
$$

where $\operatorname{Disc} \Pi_{\mu}^{\mu}(P) \equiv\left[\Pi_{\mu}^{\mu}(E+i \epsilon, \vec{p})-\Pi_{\mu}^{\mu}(E-i \epsilon, \vec{p})\right] /(2 i)$. Since the photon is a singlet under color, there is no ambiguity in how we do the analytic continuation for the photon energy. We have also used the fact that the spectral function is real. When the $Q^{a}$ 's vanish, this discontinuity is the same as the imaginary part of the retarded self-energy. When the $Q^{a} \neq 0$, however, if we were to compute the imaginary part, we would also obtain contributions from the imaginary parts of the statistical distribution functions, which are complex valued. To us this is an unphysical contribution which we neglect. After all, the discontinuity is directly related to the amplitude to produce physical particles, albeit with an (imaginary) chemical potential for color.

By using the decomposition of the spectral functions, eqs. (4.40) and (4.41),

$$
\begin{aligned}
\operatorname{tr}\left[\rho_{a}^{*}(\omega, \vec{k}) \rho(\omega-E, \vec{k}-\vec{p})\right]= & 4 \pi \epsilon(\omega-E) \delta\left((P-K)^{2}\right)\left(\rho_{+a}^{*}(\omega, \vec{k})(\omega-E-k+\hat{k} \cdot \vec{p})\right. \\
& \left.+\rho_{-a}^{*}(\omega, \vec{k})(\omega-E+k-\hat{k} \cdot \vec{p})\right)
\end{aligned}
$$

where $p=|\vec{p}|$.

Since $k \ll T$, by using the assumption $p \gg T$, we find $p \gg k$. Using this and $P^{2}=0$,

$$
\epsilon(\omega-E) \delta\left((K-P)^{2}\right) \simeq-\frac{1}{2 p k} \delta\left(\cos \theta-\frac{\omega}{k}\right) .
$$

Thus,

$$
\begin{aligned}
\operatorname{Disc} \Pi_{\mu}^{\mu}(P) \simeq & +2 \sum_{f} q_{f}^{2} e^{2} \sum_{a=1}^{N} \int \frac{d^{3} k}{(2 \pi)^{2}} \int \frac{d \omega}{2 \pi}\left(\widetilde{n}_{a}(-E)-\widetilde{n}_{a}(0)\right) \frac{-1}{k} \delta\left(\cos \theta-\frac{\omega}{k}\right) \\
& \times\left(\rho_{+a}^{*}(\omega, \vec{k})\left(-1+\frac{\omega}{k}\right)+\rho_{-a}^{*}(\omega, \vec{k})\left(-1-\frac{\omega}{k}\right)\right) \\
= & -2 \sum_{f} q_{f}^{2} e^{2} \frac{1}{2 \pi} \sum_{a=1}^{N}\left(\widetilde{n}_{a}(-E)-\widetilde{n}_{a}(0)\right) \int_{0}^{\mu} d k k \int_{-k}^{k} \frac{d \omega}{2 \pi} \\
& \times\left(\rho_{+a}^{*}(\omega, \vec{k})\left(-1+\frac{\omega}{k}\right)+\rho_{-a}^{*}(\omega, \vec{k})\left(-1-\frac{\omega}{k}\right)\right)
\end{aligned}
$$

where we introduce an ultraviolet cutoff, $\mu$. In the HTL approximation, the only difference between the quark propagators in the usual perturbative regime and those in the semi-QGP is that in the latter, the quark thermal mass is color dependent. Thus we can use standard sum rules for the quark spectral densities [106] by just keeping track of the appropriate 
color indices.

$$
\begin{gathered}
\int_{-\infty}^{\infty} \frac{d \omega}{2 \pi} \rho_{ \pm a}^{*}(\omega, \vec{k})=1, \\
\int_{-\infty}^{\infty} \frac{d \omega}{2 \pi} \omega \rho_{ \pm a}^{*}(\omega, \vec{k})= \pm k .
\end{gathered}
$$

Using the spectral functions in the time-like region, eq. (4.42), we obtain

$$
\begin{aligned}
\operatorname{Disc} \Pi_{\mu}^{\mu}(P) \simeq & +2 \sum_{f} q_{f}^{2} e^{2} \frac{1}{2 \pi} \sum_{a=1}^{N}\left(\widetilde{n}_{a}(-E)-\widetilde{n}_{a}(0)\right) \\
& \times \int_{0}^{\mu} d k k 2\left[Z_{+a}(k)\left(-1+\frac{\omega_{+a}(k)}{k}\right)+Z_{-a}(k)\left(-1-\frac{\omega_{-a}(k)}{k}\right)\right] \\
\simeq & +2 \sum_{f} q_{f}^{2} e^{2} \frac{1}{2 \pi} \sum_{a=1}^{N}\left(\widetilde{n}_{a}(-E)-\widetilde{n}_{a}(0)\right) \\
& \times\left[\mu\left(\omega_{-a}(\mu)-\omega_{+a}(\mu)\right)+2 \int_{0}^{\mu} d k\left(\omega_{+a}(k)-\omega_{-a}(k)\right)\right]
\end{aligned}
$$

where we have used [106]

$$
\left(\omega_{ \pm} \mp k\right)\left(\omega_{ \pm}^{2}-k^{2}\right) \frac{1}{m_{\mathrm{qk}}^{2}}=\omega_{ \pm}-k \frac{d \omega_{ \pm}}{d k}
$$

The wave function constants and the mass shells are functions of the color index, $a$, but we suppress this index for now to make it easier to read. By using the asymptotic form for the mass shells at hard momenta, $k \gg g T$,

$$
\begin{aligned}
& \omega_{+} \simeq k+\frac{m_{\mathrm{qk}}^{2}}{k}, \\
& \omega_{-} \simeq k,
\end{aligned}
$$

we get

$$
\begin{aligned}
\operatorname{Disc} \Pi_{\mu}^{\mu}(P) \simeq+2 \sum_{f} q_{f}^{2} e^{2} \frac{1}{2 \pi} & \sum_{a=1}^{N} m_{\mathrm{qk} a}^{2}\left(\widetilde{n}_{a}(-E)-\widetilde{n}_{a}(0)\right) \\
\times & {\left[-1+2 \int_{0}^{\mu} d k \frac{\omega_{+a}(k)-\omega_{-a}(k)}{m_{\mathrm{qk}}^{2} a}\right] . }
\end{aligned}
$$

We now make the dependence of the thermal quark mass on the color index a manifest again.

Evaluating the integral by using eqs. (4.56) and (4.57) at the leading-log accuracy,

$$
\operatorname{Disc} \Pi_{\mu}^{\mu}(P) \simeq+2 \sum_{f} q_{f}^{2} e^{2} \frac{1}{2 \pi} \sum_{a=1}^{N} m_{\mathrm{qk} a}^{2}\left(\widetilde{n}_{a}(-E)-\widetilde{n}_{a}(0)\right) \ln \left(\frac{\mu^{2}}{g^{2} T^{2}}\right) .
$$

The lower limit of the integral comes from $k \sim g T$, in which eqs. (4.56) and (4.57) becomes unreliable. 
To leading logarithmic order, then, the $Q^{a}$ 's only enter through the statistical distribution functions of the quarks, and the quark thermal mass. By using eqs. (3.3) and (4.1), the contribution to the production rate for photons from soft quarks is found to be

$$
p \frac{d \Gamma}{d^{3} p}=\left.f_{\gamma}^{\mathrm{soft}}(Q) p \frac{d \Gamma}{d^{3} p}\right|_{\mathrm{pQGP}} .
$$

The result in the perturbative QGP [6] is

$$
\left.p \frac{d \Gamma}{d^{3} p}\right|_{\mathrm{pQGP}}=-\sum_{f} q_{f}^{2} \frac{1}{8} \alpha_{\mathrm{em}} \alpha_{s} \frac{T^{2}}{\pi^{2}}\left(N^{2}-1\right)\left(\frac{\widetilde{n}(-p)-1 / 2}{1-e^{p / T}}\right) \ln \left(\frac{\mu^{2}}{g^{2} T^{2}}\right) .
$$

In the semi-QGP, this is modified by a $Q$-dependent factor,

$$
f_{\gamma}^{\mathrm{soft}}(Q)=\frac{1}{N} \frac{\sum_{a=1}^{N} m_{\mathrm{qk} a}^{2}\left(\widetilde{n}_{a}(-p)-\widetilde{n}_{a}(0)\right)}{m_{\mathrm{qk}}^{2}(\widetilde{n}(-p)-\widetilde{n}(0))},
$$

where $m_{\mathrm{qk}}$ is the thermal mass when $Q^{a}=0$, whose expression will be written later.

To evaluate the photon production rate in the semi-QGP, we need the explicit form of the thermal quark mass when $Q^{a} \neq 0$. From ref. [75],

$$
m_{\mathrm{qk} a}^{2}=\frac{g^{2}}{24}\left(\sum_{b=1}^{N}\left(\mathcal{A}\left(Q^{a}-Q^{b}\right)-\widetilde{\mathcal{A}}\left(Q^{b}\right)\right)-\frac{1}{N}\left(\mathcal{A}(0)-\widetilde{\mathcal{A}}\left(Q^{a}\right)\right)\right) .
$$

The function $\mathcal{A}(Q)$ is given by

$$
\mathcal{A}(Q)=\frac{3}{\pi^{2}} \int_{0}^{\infty} d E E\left(\frac{1}{e^{(E+i Q) / T}-1}+\frac{1}{e^{(E-i Q) / T}-1}\right),
$$

and $\widetilde{\mathcal{A}}(Q) \equiv \mathcal{A}(Q+\pi T)$. Note that $\mathcal{A}(Q)$ is an even function of $Q$.

Our definition of $\mathcal{A}(Q)$ differs by $T^{2}$ from that in ref. [75], which we do to emphasize the physics in the following section, eqs. (4.78) and (4.79). Also for the purposes of this discussion to follow, we note that in eq. (4.62) the terms involving $\sum_{b} \mathcal{A}\left(Q^{a}-Q^{b}\right)$ and $\mathcal{A}(0)$ are from the gluon distribution functions, while $\widetilde{\mathcal{A}}\left(Q^{b}\right)$ and $\widetilde{\mathcal{A}}\left(Q^{a}\right)$ are from the quark distribution functions.

In the perturbative QGP, the thermal quark mass squared is

$$
m_{\mathrm{qk}}^{2}=\frac{g^{2}}{24}\left(N-\frac{1}{N}\right) T^{2}\left(1-\left(-\frac{1}{2}\right)\right)=\left(\frac{N^{2}-1}{2 N}\right) \frac{g^{2} T^{2}}{8} .
$$

In the first expression the 1 is from the gluon distribution functions, while the $+1 / 2$ is from the quark distribution functions.

It is direct to evaluate $\mathcal{A}\left(Q^{a}\right)$ in terms of the dimensionless variable $q^{a}=Q^{a} /(2 \pi T)$, eq. (2.6),

$$
\mathcal{A}(Q)=\left(1-6|q|_{\bmod 1}\left(1-|q|_{\bmod 1}\right)\right) T^{2} .
$$

While nominally a quadratic polynomial in $q$, some care must be taken in using this expression. Only the absolute value of $q$ enters because by construction eq. (4.63) is even in 
$Q$. Secondly, $q$ is defined modulo one, since only $\exp \left( \pm 2 \pi i q^{a}\right)$ enters into the Bose-Einstein distribution functions in eq. (4.63), so the $q^{a}$ are manifestly periodic variables.

Equation (4.61) can be simplified for large photon energy $p \gg T$. In this case, $\widetilde{n}_{a}(-p) \sim$ $\widetilde{n}(-p) \sim 1$, independent of $q$. We further make use of the fact that all $Q$ 's pair up as in eq. (2.8) and the corresponding thermal quark masses are identical for the components in the pair, $m_{\mathrm{qk}}^{2} a=m_{\mathrm{qk}}^{2} N+1-a$. Consequently, we have

$$
\sum_{a=1}^{N} m_{\mathrm{qk} a}^{2}\left(\widetilde{n}_{a}(-p)-\widetilde{n}_{a}(0)\right) \simeq \sum_{a=1}^{N / 2} m_{\mathrm{qk} a}^{2}\left(2-\widetilde{n}_{a}(0)-\widetilde{n}_{N+1-a}(0)\right)=\sum_{a=1}^{N} m_{\mathrm{qk} a}^{2}(1-\widetilde{n}(0)) .
$$

This allows us to express $f_{\gamma}^{\text {soft }}(Q)$ as the ratio of average thermal quark mass squared when $Q^{a} \neq 0$ to the perturbative thermal quark mass:

$$
f_{\gamma}^{\mathrm{soft}}(Q) \simeq \frac{1}{N} \frac{\sum_{a} m_{\mathrm{qk}}^{2} a}{m_{\mathrm{qk}}^{2}}
$$

We note that eq. (4.67) is derived assuming an even $N$. The conclusion holds for odd $N$ also.

For three colors, taking the eigenvalues as in eq. (2.11), the components of thermal mass read

$$
\begin{gathered}
m_{\mathrm{qk} 1}^{2}=m_{\mathrm{qk} 3}^{2}=\frac{g^{2} T^{2}}{6}\left(1-\frac{9}{2} q+5 q^{2}\right), \\
m_{\mathrm{qk} 2}^{2}=\frac{g^{2} T^{2}}{6}(1-3 q) .
\end{gathered}
$$

The suppression factor is then

$$
f_{\gamma}^{\mathrm{soft}}(Q)=\frac{1}{3}\left[(1-3 q)+\left(1-\frac{9}{2} q+5 q^{2}\right) \frac{\widetilde{n}_{1}(-p)-\widetilde{n}_{1}(0)+\widetilde{n}_{3}(-p)-\widetilde{n}_{3}(0)}{(\widetilde{n}(-p)-\widetilde{n}(0))}\right] .
$$

For large energy, we obtain a simple polynomial in $q$,

$$
f_{\gamma}^{\text {soft }}(Q) \simeq \frac{1}{3}\left[(1-3 q)+2\left(1-\frac{9}{2} q+5 q^{2}\right)\right]=1-4 q+\frac{10}{3} q^{2},
$$

which agrees with the suppression factor for the hard contribution, $f_{\gamma}(Q)$. Altogether, the photon production rate from soft momentum exchange is

$$
p \frac{d \Gamma}{d^{3} p}=\sum_{f} q_{f}^{2} \frac{1}{2} \alpha_{\mathrm{em}} \alpha_{s} \frac{T^{2}}{\pi^{2}} e^{-p / T} \ln \left(\frac{\mu^{2}}{g^{2} T^{2}}\right) f_{\gamma}(Q) .
$$

Comparing the hard contribution in eq. (4.35) to the soft contribution in eq. (4.70), we see that the dependence upon the momentum cutoff $\mu$ cancels. This is a nontrivial check of our computation. The sum of the two contributions is

where

$$
p \frac{d \Gamma}{d^{3} p}=\left.f_{\gamma}(Q) p \frac{d \Gamma}{d^{3} p}\right|_{\mathrm{pQGP}},
$$

$$
\left.p \frac{d \Gamma}{d^{3} p}\right|_{\mathrm{pQGP}}=\sum_{f} q_{f}^{2} \frac{1}{2} \alpha_{\mathrm{em}} \alpha_{s} \frac{T^{2}}{\pi^{2}} e^{-p / T} \ln \left(\frac{p}{g^{2} T}\right) .
$$

We can extract $Q$ from lattice results of Polyakov loop and obtain $f(Q)$ as a function of the temperature. The result is shown in figure 7 . 


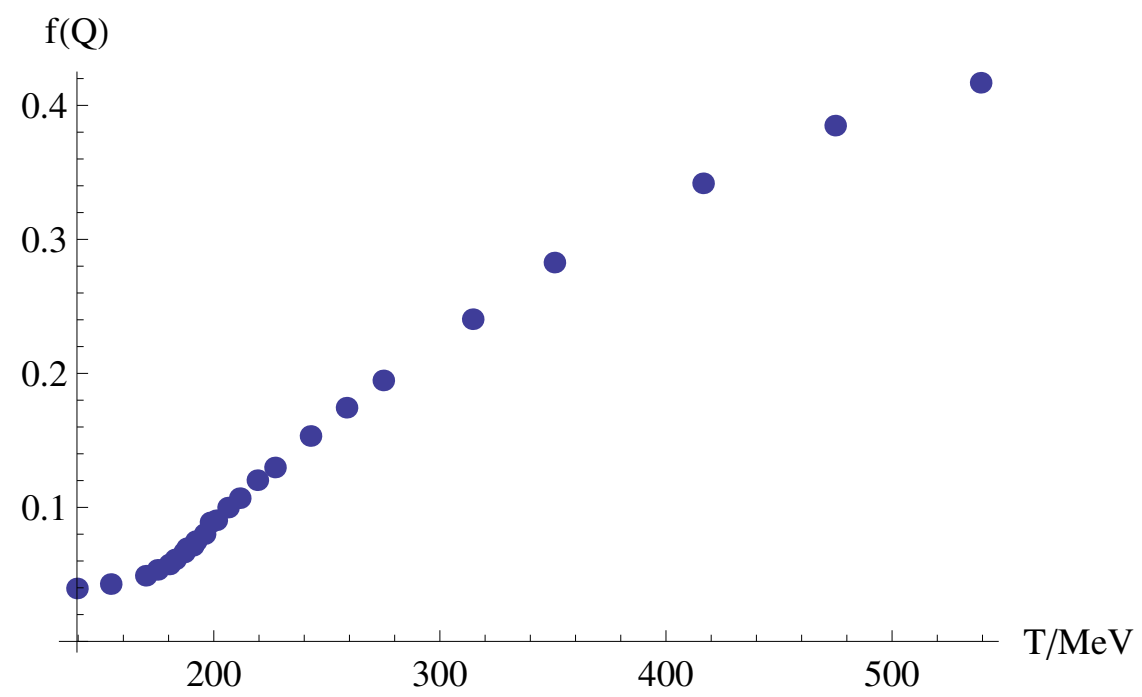

Figure 7. The suppression factor $f_{\gamma}(Q)$ in eq. (4.69) versus temperature.

\subsection{Why so few photons are produced in the semi-QGP}

For dilepton production we found a moderate enhancement near $T_{c}$. In contrast, figure 7 shows that photon production is strongly suppressed in the semi-QGP, versus the perturbative QGP. To understand the suppression of photons, as in section 3.2 it helps to generalize the computation to an arbitrary number of colors. In the calculation of the contribution from the Compton scattering, the following product of the distribution function appears, as was discussed in section 4.3:

$$
\frac{1}{N^{2}} \sum_{b, c=1}^{N} e^{-\left(E_{1}-i Q_{b}\right) / T} e^{-\left(E_{2}-i Q_{c}+i Q_{b}\right) / T}\left(1-\widetilde{n}_{c}\left(E^{\prime}\right)\right) .
$$

Here the Boltzmann approximation was applied to the initial state, and we took the large$N$ limit, in which we ignore the second term of $\left(T^{d c}\right)_{b e}$ (eq. (2.3)) appearing in the matrix element squared. The factor $1 / N^{2}$ was multiplied for normalization. The quantity above becomes

$$
\frac{1}{N} \sum_{c=1}^{N} e^{-E_{1} / T} e^{-\left(E_{2}-i Q_{c}\right) / T}\left(1-\widetilde{n}_{c}\left(E^{\prime}\right)\right)
$$

after partial cancellation of the phase of the distribution functions in the initial state. Here we note that this cancellation is not complete unlike the dilepton case: the phase $i Q_{c} / T$ still remains in the present case while the phase completely cancels for dilepton production in the Boltzmann approximation [98]. By performing the sum as in the dilepton case and using eq. (2.10), this expression can be rewritten as

$$
e^{-E / T} \widetilde{n}\left(N E^{\prime}\right)
$$

in the confined phase. We see that this expression vanishes in the $N \rightarrow \infty$ limit, unlike the dilepton case. The origin of this behavior can be tracked to the fact that the cancellation of 
the phase of the distribution functions for the initial state is only partial, and not complete. This is because that the initial state for photon production is not a color singlet, as it is for dilepton production.

For the contribution from pair annihilation, the product of the distribution functions is, in the confined phase and the large- $N$ limit, again $e^{-E / T} n\left(N E^{\prime}\right)$. We note that previously [98], we gave a similar but simpler analysis, using the Boltzmann approximation to both the final as well as the initial state.

Next, let us discuss more quantitative point: the origin of the $1 / N^{2}$ dependence of the suppression factor in the confined phase. For hard photons, with $E \gg T$, we have shown that the ratio of photon production in the semi-QGP, to that in the perturbative QGP, is just the ratio of the thermal quark masses squared, of course summed over color:

$$
f_{\gamma}(Q)=\frac{1}{m_{\mathrm{qk}}^{2}} \frac{1}{N} \sum_{a=1}^{N} m_{\mathrm{qk} a}^{2}
$$

This result is not surprising, as the photon production rate is usually written [6-11] as proportional to the thermal quark mass squared. In the perturbative QGP this is somewhat trivial, however, as photon production is naturally proportional to $\sim e^{2} g^{2} T^{2}$. This relation is less trivial in the semi-QGP, since then the thermal quark mass is a function of the $Q^{a}$ 's. Of course eq. (4.76) holds only to the order at which we compute, which is leading logarithmic order.

To illustrate how large photon suppression can be, we take the most extreme case, the confined phase. It is most useful to use the form of $\mathcal{A}(Q)$ in eq. (4.63), as an integral over the energy, $E$. The gluon distribution enters as

$$
\frac{1}{N^{2}} \sum_{a, b=1}^{N} \mathcal{A}\left(Q^{a}-Q^{b}\right)=\frac{6}{\pi^{2}} \int_{0}^{\infty} d E E \frac{1}{N^{2}} \sum_{a, b=1}^{N} \frac{1}{e^{\left(E-i\left(Q^{a}-Q^{b}\right)\right) / T}-1} .
$$

In the perturbative QGP, $Q^{a}=0$, this integral is $\mathcal{A}(0)=T^{2}$. In the confined phase, we use eq. (2.18) to obtain

$$
\frac{1}{N^{2}} \sum_{a, b=1}^{N} \mathcal{A}\left(Q_{\mathrm{conf}}^{a}-Q_{\mathrm{conf}}^{b}\right)=\frac{6}{\pi^{2}} \int_{0}^{\infty} d E E \frac{1}{e^{N E / T}-1}=\frac{T^{2}}{N^{2}}
$$

Notice that the integral over $E$ is exactly the same as when $Q^{a}=0$. The only difference is that because only loops which are multiples of $N$ enter, the energy enters not as $E / T$, but as $N E / T$. Hence in the confined phase we can replace $T$ by $T / N$ : as the integral is $\sim T^{2}$, this term is suppressed by $1 / N^{2}$ relative to that in the perturbative QGP.

From eq. (4.78), we see that the terms involving the gluon distribution function in the thermal quark mass squared, eq. (4.62), cancel identically. This leaves only the terms from the quark distribution functions, which are functions of the color index $a$. However, photon 
production only depends only upon the sum over colors, eq. (4.76), and so we compute

$$
\begin{aligned}
\frac{1}{N} \sum_{b=1}^{N} \widetilde{\mathcal{A}}\left(Q_{\text {conf }}^{b}\right) & =-\frac{6}{\pi^{2}} \int_{0}^{\infty} d E E \frac{1}{N} \sum_{b=1}^{N} \frac{1}{e^{\left(E-i Q_{\text {conf }}^{b}\right) / T}+1} \\
& =-\frac{6}{\pi^{2}} \int_{0}^{\infty} d E E \frac{1}{e^{N E / T}+1}=-\frac{T^{2}}{2 N^{2}}
\end{aligned}
$$

by using eq. (2.17). Again, this result is precisely $1 / N^{2}$ times the result for $Q^{a}=0$. We thus find that in the confined phase, the square of the thermal quark masses, summed over color, is

$$
\frac{1}{N} \sum_{a=1}^{N} m_{\mathrm{qk}}^{2}\left(Q_{\text {conf }}\right)=\left(\frac{N^{2}-1}{2 N}\right) \frac{g^{2} T^{2}}{24} \frac{1}{N^{2}} .
$$

Comparing to the thermal quark mass in the perturbative QGP, eq. (4.64), we obtain

$$
f_{\gamma}\left(Q_{\text {conf }}\right)=\frac{1}{3 N^{2}}
$$

The coefficient of $1 / 3$ arises as follows. As discussed following eq. (4.64), for the thermal quark mass squared in the perturbative QGP, the gluon terms contribute two thirds (the 1 ), and the quarks, one third (the $1 / 2$ ). In the confined vacuum the gluon distributions cancel identically, while the quark terms are precisely $1 / N^{2}$ times that for $Q^{a}=0$, or $1 /\left(3 N^{2}\right)$ in all.

This shows that photon production is strongly suppressed in the confined phase, by $\sim 1 / N^{2}$. Because the coefficient is small, $=1 / 3$, even for three colors the suppression is significant, $=1 / 27$. This is why the suppression in figure 7 is so dramatic.

The above analysis applies to the soft contribution to photon production. It can also be computed from the hard contribution to photon production, since the suppression factor is common. As demonstrated in section 4.3, there are two contributions. That from Compton scattering is given in eq. (4.30), where by definition, $f_{\text {Comp }}(0)=1$ in the perturbative QGP. To compute its value in the confined phase of the pure gauge theory, we remember that the only nonzero loops are those which wrap around a multiple of $N$ times, eq. (2.10). Hence

$$
f_{\text {Comp }}\left(Q_{\text {conf }}\right)=\frac{12}{\pi^{2}} \sum_{n=1}^{\infty} \frac{(-1)^{n+1}}{n^{2}} \ell_{n}\left(Q_{\text {conf }}\right)=\frac{12}{\pi^{2}} \sum_{j=1}^{\infty} \frac{(-1)^{j+1}}{(j N)^{2}}=\frac{1}{N^{2}} .
$$

The contribution of pair annihilation is given by $f_{\text {pair }}(Q)$ in eq. (4.34), where $f_{\text {pair }}(0)=1$. In the confined phase,

$$
f_{\text {pair }}\left(Q_{\text {conf }}\right)=\frac{1}{N^{2}-1} \frac{6}{\pi^{2}}\left(N^{2} \sum_{j=1}^{\infty} \frac{1}{(j N)^{2}}-\sum_{n=1}^{\infty} \frac{1}{n^{2}}\right)=0,
$$

and the contribution from pair annihilation vanishes identically.

In the confined phase, then, the hard part of photon production only receives a contribution from Compton scattering. From eq. (4.36), relative to the perturbative QGP photon 


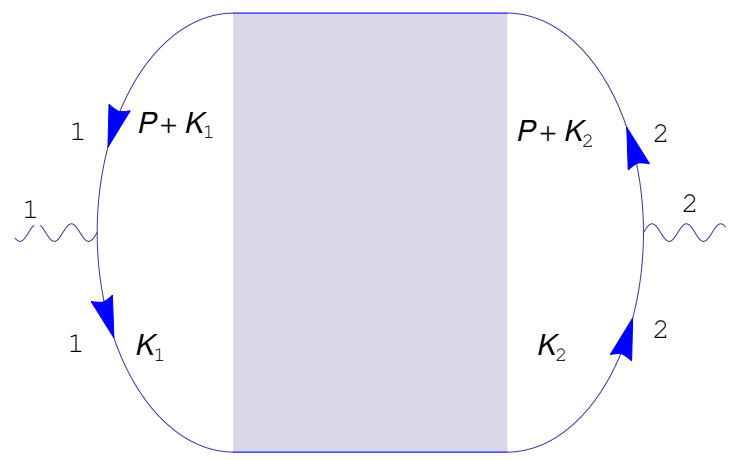

Figure 8. The diagram of four point function with labels indicating fields insertions on different branches of the Keldysh contour.

production in the semi-QGP is one third the sum of Compton scattering, plus equal contributions from pair annihilation in the $t$ and $u$ channels. Since pair annihilation vanishes in the confined phase, eq. (4.83), and the contribution from the Compton scattering is just $1 / N^{2}$ times that of the perturbative QGP, eq. (4.82), in all we obtain a relative suppression factor of $1 /\left(3 N^{2}\right)$, eq. (4.81).

\section{Collinear rate}

\subsection{Review of AMY's calculation of photon production}

\subsubsection{Photon self-energy in RA basis}

First we recapitulate the detailed analysis by Arnold, Moore, and Yaffe (AMY) [9-11] for the collinear contribution to the photon production, in the case that $Q_{a}=0$. We start with the expression for differential photon emission rate, eq. (4.1). In the $1 / 2$ basis in the real time formalism, $W_{\mu \nu}$ is given by

$$
\begin{aligned}
W_{\mu \nu}= & e^{2} \sum_{f} q_{f}^{2} \int \frac{d^{4} K_{1}}{(2 \pi)^{4}} \int \frac{d^{4} K_{2}}{(2 \pi)^{4}}\left(P+2 K_{1}\right)_{\mu}\left(P+2 K_{2}\right)_{\nu} \\
& \times G_{1122}\left(-K_{1}, P+K_{1},-P-K_{2}, K_{2}\right) .
\end{aligned}
$$

As will be justified in the next subsection, $\left(P+2 K_{1}\right)_{\mu}$ and $\left(P+2 K_{2}\right)_{\nu}$ come from quarkphoton vertices. $G_{1122}\left(-K_{1}, P+K_{1},-P-K_{2}, K_{2}\right)$ is the Fourier transform of the fourpoint function $G_{1122}\left(x_{1}, x_{2}, y_{1}, y_{2}\right)$. The labels 1,2 distinguish different field insertions on the Keldysh contour. Figure 8 summarizes the field labeling and momenta flow, with convention that the momenta flow from right to left in propagators. It is easier to calculate the four-point function in the RA basis, which is defined for quarks and gluons as

$$
\begin{array}{ll}
\psi_{R}=\frac{\psi_{1}+\psi_{2}}{2}, & \psi_{A}=\psi_{1}-\psi_{2} ; \\
A_{R}^{\mu}=\frac{A_{1}^{\mu}+A_{2}^{\mu}}{2}, & A_{A}^{\mu}=A_{1}^{\mu}-A_{2}^{\mu} .
\end{array}
$$




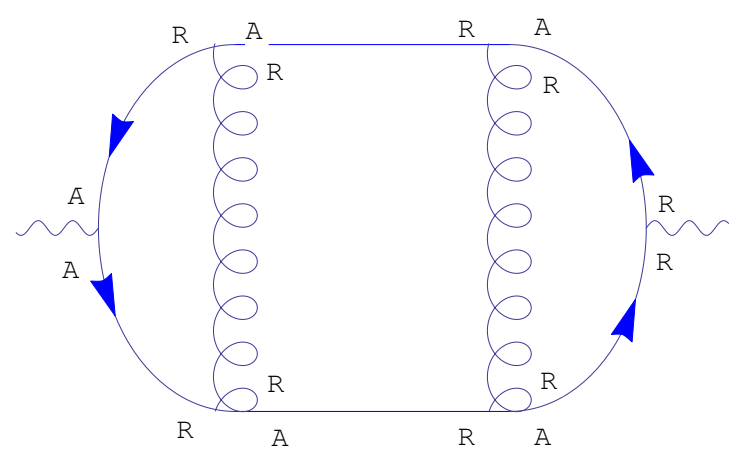

Figure 9. The four point function $G_{A A R R}$ with labels of all internal lines uniquely fixed in $R A$ basis.

In this basis, $G_{R A}$ and $G_{A R}$ correspond to the retarded and advanced propagators, respectively. The propagator $G_{A A}$ vanishes, while vertices with an odd number of $\mathrm{R}$ indices vanish. The latter is true for quark-gluon vertices only, but only these are relevant to the calculation of the collinear rate. To perform the calculation in the RA basis, we need to decompose $G_{1122}$ in terms of four-point functions. While there are in total 16 four-point functions, only 7 of them are independent [107]. The decomposition into an independent set has been done for neutral scalar in ref. [107]. It is easily generalized to the case of fermions with $\mu=0$ as

$$
\begin{aligned}
G_{1122}= & \alpha_{1} G_{A A R R}+\alpha_{2} G_{A A A R}+\alpha_{3} G_{A A R A}+\alpha_{4} G_{A R A A} \\
& +\alpha_{5} G_{R A A A}+\alpha_{6} G_{A R R A}+\alpha_{7} G_{A R A R} \\
& +\beta_{1} G_{A A R R}^{*}+\beta_{2} G_{A A A R}^{*}+\beta_{3} G_{A A R A}^{*}+\beta_{4} G_{A R A A}^{*} \\
& +\beta_{5} G_{R A A A}^{*}+\beta_{6} G_{A R R A}^{*}+\beta_{7} G_{A R A R}^{*} .
\end{aligned}
$$

Detailed analysis by AMY [9-11] shows that the collinear rate receives contributions only from $G_{A A R R}$. Thus the only coefficients which we need are $\alpha_{1}$ and $\beta_{1}$,

$$
\alpha_{1}=\widetilde{n}\left(p_{1}\right) \widetilde{n}\left(p_{2}\right), \beta_{1}=-\left(1-\widetilde{n}\left(p_{3}\right)\right)\left(1-\widetilde{n}\left(p_{4}\right)\right) \frac{-1+\widetilde{n}\left(p_{1}\right)+\widetilde{n}\left(p_{2}\right)}{-1+\widetilde{n}\left(p_{3}\right)+\widetilde{n}\left(p_{4}\right)} .
$$

In our case $\left(p_{1}, p_{2}, p_{3}, p_{4}\right)=\left(-K_{1}, P+K_{1},-P-K_{2}, K_{2}\right)$.

For the four-point function $G_{A A R R}$, the RA labeling is uniquely fixed as in figure 9 . The contribution to the collinear regime arises from the kinematic regime where hard quark in the loop is nearly collinear with the photon: $k^{0} \simeq k_{\|} \gtrsim T, k_{\perp} \sim g T$, with $\|$ and $\perp$ defined with respect to photon momentum $p$. The gluon exchanged between the quark lines are soft: $q^{0} \sim g T, q \sim g T$. From the collinear scattering of quarks and gluons, the energy of the quarks remain unmodified at order $T$. With this kinematic simplification, eq. (5.5) reduces to

$$
\alpha_{1} \simeq \beta_{1} \simeq \widetilde{n}\left(k_{\|}+p\right)\left(1-\widetilde{n}\left(k_{\|}\right)\right)
$$

and therefore,

$$
G_{1122}=2 \alpha_{1} \operatorname{Re} G_{A A R R}
$$




\subsubsection{Reduction of spinor structure}

To proceed, we need to see how collinear enhancement works. To do so, consider the convolution of two quark propagators, which enters as a unit upon inserting an additional gluon scattering into $G_{A A R R}$ :

$$
\int \frac{d k^{0}}{2 \pi} S_{A R}(P+K) S_{R A}(K)
$$

$S_{A R}$ and $S_{R A}$ are advanced and retarded dressed quark propagators:

$$
-S=\frac{1}{\not k-\not}=\frac{1}{2} \frac{\gamma^{0}-\vec{\gamma} \cdot \hat{k}}{A_{0}-A_{s}}+\frac{1}{2} \frac{\gamma^{0}+\vec{\gamma} \cdot \hat{k}}{A_{0}+A_{s}}
$$

where

$$
A_{0}=k^{0}-\Sigma^{0}, A_{s}=|\vec{k}-\vec{\Sigma}| .
$$

Here $\Sigma=\Sigma^{\mu} \gamma_{\mu}$ is the retarded or advanced quark self-energy, and $\Sigma^{\mu}$ does not have a spinor structure. Note that due to rotational symmetry, $\vec{k} \| \vec{\Sigma}$, so $A_{s}=|k-\Sigma|$. The advanced and retarded propagators differ only in the sign of the damping rate, which corresponds to the imaginary part of self-energy $\Sigma$. Both $S_{A R}$ and $S_{R A}$ have two poles with positive and negative energies. The collinear enhancement occurs when two poles coming from the two propagators pinch the real axis of $k_{0}$ plane. Thus, it suffices to consider the pole contribution:

$$
-S(k) \simeq\left\{\begin{array}{l}
\frac{1}{2} \frac{\gamma^{0}-\vec{\gamma} \cdot \hat{k}}{A_{0}-A_{s}} \simeq \frac{\not k}{2 k^{0}\left(A_{0}-A_{s}\right)} \text { for } k^{0}>0 \\
\frac{1}{2} \frac{\gamma^{0}+\vec{\gamma} \cdot \hat{k}}{A_{0}+A_{s}} \simeq \frac{\not k}{2 k^{0}\left(A_{0}+A_{s}\right)} \text { for } k^{0}<0
\end{array} .\right.
$$

It is useful to write $\not k$ in terms of spinor sums:

$$
\begin{aligned}
& \not k=\sum_{s} u_{s}(k) \bar{u}_{s}(k), \text { for } k^{0}>0, \\
& \not k=\sum_{t} v_{t}(k) \bar{v}_{t}(k), \text { for } k^{0}<0,
\end{aligned}
$$

where $u$ and $v$ refer to the spinor basis

$$
\begin{aligned}
& u=\left(\sqrt{k \cdot \sigma} \xi_{s}, \sqrt{k \cdot \bar{\sigma}} \xi_{s}\right)^{T}, \\
& v=\left(\sqrt{k \cdot \sigma} \eta_{t},-\sqrt{k \cdot \bar{\sigma}} \eta_{t}\right)^{T},
\end{aligned}
$$

with

$$
\xi_{s}=\left(\delta_{s 1}, \delta_{s 2}\right)^{T}, \eta_{t}=\left(\delta_{t 1}, \delta_{t 2}\right)^{T}, \quad s, t=1,2 .
$$

Here, $\sigma_{\mu}=\left(1, \sigma^{i}\right), \bar{\sigma}_{\mu}=\left(1,-\sigma^{i}\right)$ with Pauli matrices $\sigma^{i}$. Note that $k^{0}, k \gtrsim T$ and $\Sigma \sim g T$, so we can take

$$
A_{0}-A_{s} \simeq k^{0} \pm \frac{i}{2} \Gamma_{k}-E_{k}, \quad A_{0}+A_{s} \simeq k^{0} \pm \frac{i}{2} \Gamma_{k}+E_{k}, \quad E_{k}=\sqrt{k^{2}+m^{2}} .
$$

The asymptotic thermal mass $m$ and damping rate $\Gamma_{k} / 2$ are of order $m \sim g T$ and $\Gamma_{k} \sim$ $g^{2} T$, and the explicit expressions of these quantities will be given later. For the retarded 
(advanced) propagator, we take the positive (negative) sign, respectively. It is not difficult to find that the pinching of poles occurs when $k^{0} \simeq k_{\|}$, with $k_{\|}$defined with respect to photon momentum $p$. With the approximation in eqs. (5.15) and (5.8) evaluates to

$$
\left.\int \frac{d k^{0}}{2 \pi} S_{A R}(P+K) S_{R A}(K) \simeq \frac{(P+K) K}{4 k_{\|}\left(k_{\|}+p\right)(\Gamma+i \delta E)}\right|_{k^{0}=k_{\|}},
$$

where

$$
\Gamma \equiv \frac{1}{2}\left(\Gamma_{k}+\Gamma_{k+p}\right), \delta E \equiv E_{k} \operatorname{sgn}\left(k_{\|}\right)+p-E_{k+p} \operatorname{sgn}\left(k_{\|}+p\right) .
$$

Note that $P+K$ and $K$ in the numerator of eq. (5.16) carry independent spinor indices, which are to be contracted with quark-gluon and quark-photon vertices. Contracting each quark-gluon vertex with two spinors from the propagators joining it, with eq. (5.12) there is one of two situations, depending on the sign of $k^{0}$. Since

$$
\bar{u}_{t}(K) \gamma^{\mu} u_{s}(K)=2 K^{\mu} \delta_{t s} \quad, \quad \bar{v}_{t}(K) \gamma^{\mu} v_{s}(K)=2 K^{\mu} \delta_{t s},
$$

each gives the same result. We have neglected the momentum of the exchanged soft gluon $Q$, since it is negligible compared with $K$. Cross terms between $u$ and $v$ are not allowed because multiple scatterings with soft gluons do not change the sign of $k^{0}$. We have not included the coupling constant $g$ and color factors, which will be discussed separately in the next subsection. According to eq. (5.18), each quark-gluon vertex gives rises to $2 K^{\mu}$, while maintaining the quark's chirality.

Now consider the quark-photon vertex, contracting the left/right quark-photon vertex with two spinors from the propagators joining them. As an example, consider $k^{0}>0$ :

$$
\bar{u}_{t}(K) \gamma^{\mu} u_{s}(K+P), \quad \bar{u}_{s}(K+Q+P) \gamma^{\nu} u_{t}(K+Q) .
$$

Summing over spinor indices and (transverse) photon polarizations, after some algebra $[9$, 11] we obtain

$$
\begin{aligned}
\sum_{s, t=1,2} \sum_{i=\perp} \bar{u}_{t}(K) \gamma^{i} u_{s}(K+P) \bar{u}_{s}(K+Q+P) \gamma^{i} u_{t}(K+Q) \\
=4 k^{0}\left(k^{0}+p\right) k_{\perp} \cdot\left(k_{\perp}+q_{\perp}\right)\left(\frac{\left(k^{0}\right)^{2}+\left(k^{0}+p\right)^{2}}{\left(k^{0}\right)^{2}\left(k^{0}+p\right)^{2}}\right) \\
\simeq 4 k_{\perp} \cdot\left(k_{\perp}+q_{\perp}\right)\left(\frac{k_{\|}^{2}+\left(k_{\|}+p\right)^{2}}{k_{\|}\left(k_{\|}+p\right)}\right)
\end{aligned}
$$

The other cases are similar, with the same result as eq. (5.20). Note that by definition $p_{\perp}=0$, so we can write eq. (5.20) as

$$
\begin{aligned}
& \sum_{s, t=1,2} \sum_{i=\perp} \bar{u}_{t}(K) \gamma^{i} u_{s}(K+P) \bar{u}_{s}(K+Q+P) \gamma^{i} u_{t}(K+Q) \\
&=\left(\frac{k_{\|}^{2}+\left(k_{\|}+p\right)^{2}}{k_{\|}\left(k_{\|}+p\right)}\right) \sum_{i=\perp} \epsilon_{\mu}^{i} \epsilon_{\nu}^{i}(2 K+P)^{\mu}(2 K+2 Q+P)^{\nu} .
\end{aligned}
$$




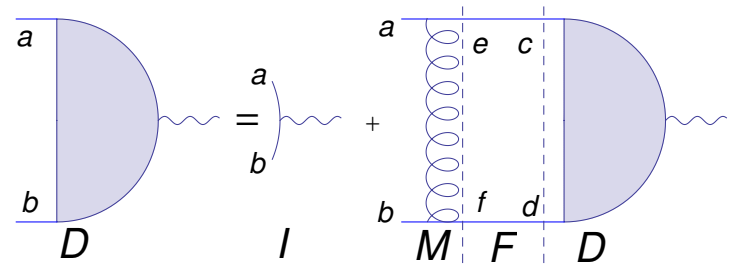

Figure 10. The diagrammatic equation in terms of graphical elements $D, I, M$ and $F$.

Apart from an overall factor $\left(k_{\|}^{2}+\left(k_{\|}+p\right)^{2}\right) /\left(k_{\|}\left(k_{\|}+p\right)\right)$, eq. (5.21) allows us to interpret $(2 K+P)^{\mu}$ and $(2 K+2 Q+P)^{\nu}$ as quark-photon vertices on the left and right of self-energy diagrams. We have thus shown in eqs. (5.18) and (5.21) that quark-gluon and quark-photon vertices can be simplified as $2\left(K_{1}+K_{2}\right)^{\mu}$ with $K_{1}$ and $K_{2}$ being the incoming and outgoing momenta of quarks. We note that $\sum_{i=\perp} \epsilon_{\mu}^{i} \epsilon_{\nu}^{i}=-g_{\mu \nu}$ as in eq. (4.1).

\subsubsection{Color structure in the double line basis}

The color structure of the gluon propagator is given by $P_{c d}^{a b}$, as in eq. (5.44). Thus the color sum which appears when a gluon propagator is sandwiched between two quark-gluon vertices is

$$
\left(T^{a b}\right)_{e f} P_{c d}^{a b}\left(T^{d c}\right)_{g h}=\frac{1}{2} P_{e f}^{h g}
$$

This can be simplified further by noting that in the photon self-energy, eq. (5.22) is sandwiched with the quark-photon vertex in the vertices which are all the way to the left or all the way to the right. Starting from the left hand side gives

$$
\frac{1}{2} \delta_{h}^{e} P_{e f}^{h g}=\frac{1}{2}\left(N-\frac{1}{N}\right) \delta_{g}^{f}=C_{F} \delta_{g}^{f}
$$

where $C_{F}=\left(N^{2}-1\right) /(2 N)$ is the quadratic Casimir for the fundamental representation. If this is iterated further, each quark-gluon vertex preserves the Kronecker delta in color, and generates an additional factor of $C_{F}$. After the last quark-gluon scattering, the delta function is color is contracted with the right most quark-photon vertex, giving an overall factor of $N$.

\subsubsection{Resummation of infinite self-energy diagrams}

We next resum diagrams with arbitrary quark-gluon scatterings. This is done by solving the integral equation illustrated in figure 10. The graphical elements are the same as those defined by Arnold, Moore, and Yaffe [9, 11], except that we use the double line notation for future convenience. The integral equation shown in figure 10 becomes

$$
D_{a b}^{\mu}(K, P)=I_{a b}^{\mu}(K, P)+\int \frac{d^{4} q}{(2 \pi)^{4}} M(K, Q, P)_{a b, e f} F(K+Q, P)_{e f, c d} D_{c d}^{\mu}(K+Q, P)
$$


The color structure can be taken as

$$
\begin{aligned}
I_{a b}^{\mu}(K, P) & =I^{\mu}(K, P) \delta_{a b}, \\
M(K, Q, P)_{a b, e f} & =\frac{1}{2 C_{F}} \mathcal{P}_{b f}^{a e} M(K, Q, P), \\
F(K+Q, P)_{e f, c d} & =\delta_{c e} \delta_{d f} F(K+Q, P), \\
D_{a b}^{\mu}(K, P) & =D^{\mu}(K, P) \delta_{a b},
\end{aligned}
$$

so that eq. (5.24) simplifies

$$
D^{\mu}(K, P)=I^{\mu}(K, P)+\int \frac{d^{4} q}{(2 \pi)^{4}} M(K, Q, P) F(K+Q, P) D^{\mu}(K+Q, P) .
$$

Note the color factor from eq. (5.23) cancels the factor of $1 /\left(2 C_{F}\right)$ in eq. (5.25).

As discussed previously, we regard the quark-gluon vertices and quark-photon vertices as $(K+Q)^{\mu}$, with $K$ and $Q$ being the incoming and outgoing momenta. As a result,

$$
I^{\mu}(K, P)=(2 K+P)^{\mu},
$$

and the function $F(K, P)$ equals

$$
\begin{aligned}
F(K, P) & =\left.(-i)^{2} G_{A R}(P+K) G_{R A}(K)\right|_{\text {pinch }} \\
& \simeq\left(\frac{-1}{4 k_{\|}\left(k_{\|}+p\right)}\right)\left(\frac{1}{\Gamma+i \delta E}\right) 4 \pi \delta\left(2 k^{0}+p-E_{k} \operatorname{sgn}\left(k_{\|}\right)-E_{k+p} \operatorname{sgn}\left(k_{\|}+p\right)\right) \\
& \simeq\left(\frac{-1}{4 k_{\|}\left(k_{\|}+p\right)}\right)\left(\frac{1}{\Gamma+i \delta E}\right) 2 \pi \delta\left(k^{0}-k_{\|}\right) .
\end{aligned}
$$

The pinching condition is enforced by the delta function. The rung of the ladder equals

$$
\begin{aligned}
M(K, Q, P) & =i g^{2} C_{F}(2 K+Q+2 P)^{\mu}(2 K+Q)^{\nu} G_{\mu \nu}^{R R}(Q) \\
& \simeq 4 i g^{2} C_{F} k_{\|}\left(k_{\|}+p\right) \widehat{P}^{\mu} \widehat{P}^{\nu} G_{\mu \nu}^{R R}(Q)
\end{aligned}
$$

with $\widehat{P}^{\mu}=(1, \hat{p})$. The Ward identity and the fact that $K^{\mu}$ is almost collinear with $P^{\mu}$ was used to simplify eq. (5.29). To further simplify eq. (5.26), we define

$$
f^{\mu}(\mathbf{k}, p) \equiv-4 k_{\|}\left(k_{\|}+p\right) \int \frac{d k^{0}}{2 \pi} F(K, P) D^{\mu}(K, P)
$$

which leads us to

$$
(\Gamma+i \delta E) f^{\mu}(\mathbf{k}, p)=(2 K+P)^{\mu}+\int \frac{d^{3} q}{(2 \pi)^{3}} C(\mathbf{q}, p) f^{\mu}(\mathbf{k}+\mathbf{q}, p),
$$

where

$$
C(\mathbf{q}, p)=g^{2} C_{F} \int \frac{d q^{0}}{2 \pi} 2 \pi \delta\left(q^{0}-q_{\|}\right)\left(-i G_{\mu \nu}^{R R}(Q) \widehat{P}^{\mu} \widehat{P}^{\nu}\right) .
$$

The delta function in eq. (5.32) again results from $\delta\left(k^{0}+q^{0}-k_{\|}-q_{\|}\right)$in $F(K+Q, P)$. We can further simplify eq. (5.31) using the explicit expression of the damping rate $\Gamma$,

$$
\Gamma_{k}=g^{2} C_{F} \int \frac{d^{3} q d q^{0}}{(2 \pi)^{4}} 2 \pi \delta\left(q^{0}-q_{\|}\right)\left(-i G_{\mu \nu}^{R R}(Q) \widehat{P}^{\mu} \widehat{P}^{\nu}\right) .
$$


As this is independent of $k, \Gamma=\left(\Gamma_{k}+\Gamma_{k+p}\right) / 2=\Gamma_{k}$. This allows us to write eq. (5.31) as

$$
i \delta E f^{\mu}(\mathbf{k}, p)=(2 K+P)^{\mu}+\int \frac{d^{3} q}{(2 \pi)^{3}} C(\mathbf{q}, p)\left[f^{\mu}(\mathbf{k}+\mathbf{q}, p)-f^{\mu}(\mathbf{k}, p)\right] .
$$

As is clear from eq. (5.21), only the transverse components of $f^{\mu}(k, p)$ are needed, so we can project eq. (5.34) onto the transverse plane,

$$
i \delta E \mathbf{f}_{\perp}(\mathbf{k}, p)=2 \mathbf{k}_{\perp}+\int \frac{d^{3} q}{(2 \pi)^{3}} C(\mathbf{q}, p)\left[\mathbf{f}_{\perp}(\mathbf{k}+\mathbf{q}, p)-\mathbf{f}_{\perp}(\mathbf{k}, p)\right] .
$$

The last element is to determine the propagator, $G_{\mu \nu}^{R R}(Q) \widehat{P}^{\mu} \widehat{P}^{\nu}$. Since $q \sim g T$, we use the HTL-resummed propagator,

$$
-i G_{\mu \nu}^{R R}(Q) \widehat{P}^{\mu} \widehat{P}^{\nu}=-\frac{2 T}{q_{0}}\left(1-\frac{q_{\|}^{2}}{q^{2}}\right) \operatorname{Im}\left(\frac{1}{Q^{2}-\Pi_{T}^{R}(Q)}-\frac{1}{Q^{2}-\Pi_{L}^{R}(Q)}\right),
$$

where we have taken Feynman gauge, and used $q_{0} \simeq q_{\|} \cdot \Pi_{L}$ and $\Pi_{T}$ are the retarded longitudinal and transverse self-energies of the gluon:

$$
\begin{aligned}
& \Pi_{L}^{R}(Q)=-\frac{Q^{2}}{q^{2}} M^{2}\left[1-\frac{q_{0}}{2 q} \ln \left(\frac{q_{0}+q}{q_{0}-q}\right)\right], \\
& \Pi_{T}^{R}(Q)=\frac{M^{2}}{2}\left[\left(\frac{q_{0}}{q}\right)^{2}-\frac{Q^{2}}{q^{2}} \frac{q_{0}}{2 q} \ln \left(\frac{q_{0}+q}{q_{0}-q}\right)\right],
\end{aligned}
$$

where the gluon Debye mass is given by

$$
M^{2}=g^{2} T^{2}\left(\frac{N}{3}+\frac{N_{f}}{6}\right) .
$$

The Wightman correlator for two electromagnetic currents can be expressed as

$$
\begin{aligned}
W^{\mu \nu} & =(-) 2 N e^{2} \sum_{f} q_{f}^{2} \alpha_{1} \operatorname{Re} \int \frac{d^{4} K}{(2 \pi)^{4}} I^{\mu}(K, P) F(K, P) D^{\nu}(K, P)\left(\frac{k_{\|}^{2}+\left(k_{\|}+p\right)^{2}}{k_{\|}\left(k_{\|}+p\right)}\right) \\
& =N e^{2} \sum_{f} q_{f}^{2} \int \frac{d^{3} k}{(2 \pi)^{3}} A\left(k_{\|}, p\right) \operatorname{Re}\left[I^{\mu}(K, P) f^{\nu}(K, P)\right]
\end{aligned}
$$

where

$$
A\left(k_{\|}, p\right)=\widetilde{n}\left(k_{\|}+p\right)\left(1-\widetilde{n}\left(k_{\|}\right)\right)\left(\frac{k_{\|}^{2}+\left(k_{\|}+p\right)^{2}}{2 k_{\|}^{2}\left(k_{\|}+p\right)^{2}}\right) .
$$

Note that an overall factor of $\left(k_{\|}^{2}+\left(k_{\|}+p\right)^{2}\right) /\left(k_{\|}\left(k_{\|}+p\right)\right)$ in eq. (5.21) is inserted into eq. (5.40) along with -1 from the fermion loop. From eq. (4.1), $W^{\mu \nu}$ is contracted with $-g_{\mu \nu}$ to give the collinear rate:

$$
p \frac{d \Gamma_{\gamma}}{d^{3} p}=\frac{\alpha_{\mathrm{em}} N \sum_{f} q_{f}^{2}}{4 \pi^{2}} \int \frac{d^{3} k}{(2 \pi)^{3}} A\left(k_{\|}, p\right) \operatorname{Re}\left[2 \mathbf{k}_{\perp} \cdot \mathbf{f}_{\perp}(k, p)\right] .
$$

Note there is an additional factor of $N$ for each color of $W^{\mu \nu}$. 


\subsection{Photon self-energy with nontrivial Polyakov loop}

\subsubsection{Quark and gluon thermal masses with background color charge}

Now we compute the modification of the results in the previous section in the presence of a nontrivial Polyakov loop. In this case, quantities like the thermal mass, the damping rate, and so on are all dependent on the background color charge. This changes the color structure of the self-energy diagrams.

The quantities relevant for the problem at hand are the quark asymptotic thermal mass and the resummed gluon propagator. The asymptotic quark thermal mass is $\sqrt{2}$ times the quark thermal mass in eq. (4.62):

$$
m_{a}^{2}=2 m_{\mathrm{qk} a}^{2} .
$$

Next we consider the HTL-resummed gluon propagator. The resummed gluon propagator consists of bare gluon propagators with arbitrary number of self-energy insertions, figure 11. The bare gluon propagator in the RA basis of the real time formalism, in the presence of background color charge, is proportional to $\mathcal{P}_{c d}^{a b}$ :

$$
\begin{aligned}
G_{R A, c d}^{\mu \nu, a b} & =\frac{g^{\mu \nu}}{\left(k^{0}+i \epsilon\right)^{2}-k^{2}} \mathcal{P}_{c d}^{a b}, & G_{A R, c d}^{\mu \nu, a b} & =\frac{g^{\mu \nu}}{\left(k^{0}-i \epsilon\right)^{2}-k^{2}} \mathcal{P}_{c d}^{a b}, \\
G_{R R, c d}^{\mu \nu, a b} & =-i \pi \epsilon\left(k^{0}\right)\left(1+2 n_{a b}\left(k^{0}\right)\right) \delta\left(K^{2}\right) g^{\mu \nu} \mathcal{P}_{c d}^{a b}, & G_{A A}^{\mu \nu} & =0 .
\end{aligned}
$$

Here we need to recall that in the analysis in the case of $Q_{a}=0$, Bose-Einstein enhancement was essentially important for the collinear contribution to be as large as the $2 \rightarrow 2$ contribution: for soft gluons with $k^{0} \sim g T, n\left(k^{0}\right) \sim 1 / g$. This is no longer true in the presence of hard background charge $Q_{a} \sim T$. The only exception is for diagonal gluons, $Q_{a}=Q_{b}$, where Bose-Einstein enhancement is still operative. Therefore, we only need the expression of the diagonal components of the gluon propagator. Thus we contract the bare gluon propagator in eq. (5.44) with $\delta_{a b}$. As a result, the soft diagonal gluon carries only one index at large $N$ : the color structure of the bare gluon propagator is

$$
\delta_{a b} \mathcal{P}_{c d}^{a b}=\delta_{a b} \delta_{c d} \mathcal{P}_{a c}
$$

where we defined a color projection operator for diagonal gluons,

$$
\mathcal{P}_{a c} \equiv \mathcal{P}_{c c}^{a a}=\delta_{a c}-\frac{1}{N}
$$

which satisfies $\mathcal{P}_{a b} \mathcal{P}_{b c}=\mathcal{P}_{a c}$. We note that the first term of the projection operator is orthogonal among diagonal gluons while the second term mixes different diagonal gluons, which is a consequence of the over completeness of the double line basis. At large $N$, eq. (5.45) becomes $\delta_{a b} \delta_{c d} \delta_{a c}$, which indicates that there is only one color index to be specified.

In terms of diagonal gluon projection operator, the resummed gluon propagator is given by the sum of the following terms:

$$
\frac{1}{Q^{2}} \mathcal{P}_{a b}+\frac{1}{Q^{2}} \mathcal{P}_{a c} \Pi_{c d} \frac{1}{Q^{2}} \mathcal{P}_{d b}+\frac{1}{Q^{2}} \mathcal{P}_{a c} \Pi_{c d} \frac{1}{Q^{2}} \mathcal{P}_{d e} \Pi_{e f} \frac{1}{Q^{2}} \mathcal{P}_{f b}+\ldots
$$


where the Lorentz indices are suppressed for the time being. Here $\Pi_{c d}$ is given by $\Pi_{c d}(Q) \equiv$ $\Pi_{c c, d d}(Q)$, with $\Pi_{c d, e f}(Q)$ being the gluon self-energy. Each color projection operator is accompanied by a momentum dependent part of the bare propagator $1 / Q^{2}$. The color structure of one-index gluon self-energy is

$$
\Pi_{a b}=\delta_{a b} F_{a}-\frac{1}{N} G_{a b} .
$$

By restoring the Lorentz indices, the gluon self-energy in the HTL approximation [76] is given by

$$
\Pi_{a b, c d}^{\mu \nu}(Q)=M_{a b, c d}^{2} \delta \Pi^{\mu \nu}
$$

Here

$$
\delta \Pi^{\mu \nu}=\left(-\delta^{0 \mu} \delta^{0 \nu}+q^{0} \int \frac{d \Omega_{q}}{4 \pi} \frac{\widehat{Q}^{\mu} \widehat{Q}^{\nu}}{Q \cdot \widehat{Q} \pm i \epsilon}\right)
$$

with $\widehat{Q} \equiv(1, \widehat{\mathbf{q}})$. The sign in the denominator is plus (minus) if the self-energy is retarded (advanced). The form of $\delta \Pi^{\mu \nu}$ is identical to that in perturbative QGP. The Polyakov loop dependence is entirely in the gluon Debye mass:

$$
\begin{aligned}
M_{a b, c d}^{2}= & \frac{g^{2}}{6}\left[\delta_{a d} \delta_{b c}\left(\sum_{e=1}^{N}\left(\mathcal{A}\left(Q^{a e}\right)+\mathcal{A}\left(Q^{e b}\right)\right)-N_{f}\left(\widetilde{\mathcal{A}}\left(Q^{a}\right)+\widetilde{\mathcal{A}}\left(Q^{b}\right)\right)\right)\right. \\
& \left.-2 \delta_{a b} \delta_{c d}\left(\mathcal{A}\left(Q^{a c}\right)-\frac{N_{f}}{N}\left(\widetilde{\mathcal{A}}\left(Q^{a}\right)+\widetilde{\mathcal{A}}\left(Q^{c}\right)-\frac{1}{N} \sum_{e=1}^{N} \widetilde{\mathcal{A}}\left(Q^{e}\right)\right)\right)\right],
\end{aligned}
$$

which for $Q_{a}=0$ reduces to eq. (5.39), namely

$$
M_{a b, c d}^{2}=g^{2} T^{2}\left(\frac{1}{3} N+\frac{1}{6} N_{f}\right) \mathcal{P}_{a b, c d} .
$$

Equation (5.51) leads us to

$$
\begin{aligned}
F_{a}^{\mu \nu} & =\frac{g^{2}}{3}\left(\sum_{e=1}^{N} \mathcal{A}\left(Q^{a e}\right)-N_{f} \widetilde{\mathcal{A}}\left(Q^{a}\right)\right) \delta \Pi^{\mu \nu}(Q), \\
G_{a b}^{\mu \nu} & =\frac{g^{2}}{3}\left(N \mathcal{A}\left(Q^{a b}\right)-N_{f}\left(\widetilde{\mathcal{A}}\left(Q^{a}\right)+\widetilde{\mathcal{A}}\left(Q^{b}\right)\right)-\sum_{e=1}^{N} \widetilde{\mathcal{A}}\left(Q^{e}\right)\right) \delta \Pi^{\mu \nu}(Q),
\end{aligned}
$$

where we have restored the Lorentz indices.

Formally the two terms in eq. (5.48) are of the same order if we regard $\delta_{a b} \sim 1 / N$. However, we show in appendix B that a naive large $N$ limit is justified. This allows us to disregard the term proportional to $G$, so that the gluon Debye mass becomes

$$
M_{a}^{2}=\frac{g^{2}}{3}\left[\sum_{e=1}^{N} \mathcal{A}\left(Q^{a e}\right)-N_{f} \widetilde{\mathcal{A}}\left(Q^{a}\right)\right],
$$




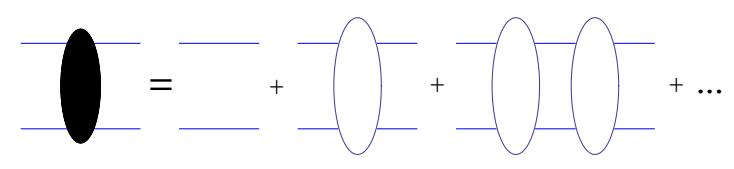

Figure 11. The resummed gluon propagator as an infinite series of propagators with arbitrary self-energy insertions. Each unfilled circle represents a self-energy insertion $\Pi$. The double line notation is used.

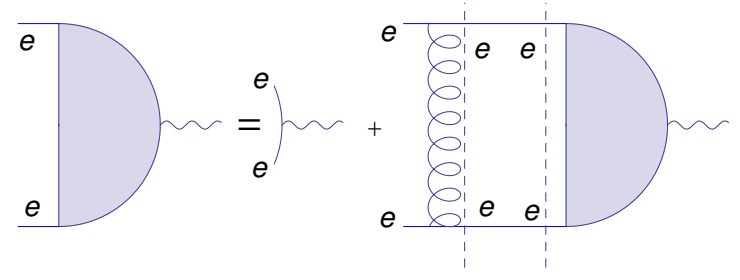

Figure 12. The diagrammatic equation for a color index $e$, in the presence of nontrivial Polyakov loop.

where $M_{a}^{2}$ is defined as $M_{a a, b b}^{2}=M_{a}^{2} \delta_{a b}$. When $Q^{a}=2 \pi T q \neq 0$, the explicit form of $M_{a}^{2}$ for $N=N_{f}=3$ are

$$
\begin{aligned}
& M_{1}^{2}=M_{3}^{2}=g^{2} T^{2}\left(\frac{3}{2}-6 q+4 q^{2}\right), \\
& M_{2}^{2}=g^{2} T^{2}\left(\frac{3}{2}-4 q+4 q^{2}\right) .
\end{aligned}
$$

\subsubsection{AMY's integral equation with one color index}

We next generalize the integral equation of AMY for a nontrivial Polyakov loop in the limit of a large number of colors. From the discussion of the previous subsection, all elements of the graph carry one color index, as in figure 12 .

The integral equation analogous to eq. (5.26) is

$$
D_{e}^{\mu}(K, P)=I_{e}^{\mu}(K, P)+\int \frac{d^{4} q}{(2 \pi)^{4}} M_{e}(K, Q, P) F_{e}(K+Q, P) D_{e}^{\mu}(K+Q, P) .
$$

Most quantities only need trivial modifications:

$$
\begin{aligned}
I_{e}^{\mu}(K, P) & =(2 K+P)^{\mu}, \\
F_{e}(K, P) & =\frac{-1}{4 k_{\|}\left(k_{\|}+p\right)} \frac{1}{\Gamma_{e}+i \delta E_{e}} 2 \pi \delta\left(k^{0}-k_{\|}\right), \\
M_{e}(K, Q, P) & =4 i g^{2} \frac{1}{2} k_{\|}\left(k_{\|}+p\right) \widehat{P}^{\mu} \widehat{P}^{\nu} G_{\mu \nu, e}^{R R}(Q) .
\end{aligned}
$$

A distinct difference is the color factor $C_{F}$ in $M_{e}$ changes to $1 / 2$. This follows from enforcing color neutrality on the soft gluon and dropping terms $1 / N$ in the gluon self-energy. Apart 
from this, the color index $e$ enters $\Gamma$ and $\delta E$ through quark asymptotic thermal mass eq. (5.43) and gluon Debye mass eq. (5.54). Explicitly,

$$
\begin{aligned}
\delta E_{e} & =E_{k} \operatorname{sgn}\left(k_{\|}\right)+p-E_{k+p} \operatorname{sgn}\left(k_{\|}+p\right) \simeq \frac{p}{2 k_{\|}\left(k_{\|}+p\right)}\left(k_{\perp}^{2}+m_{e}^{2}\right), \\
-i G_{\mu \nu e}^{R R}(Q) \widehat{P}^{\mu} \widehat{P}^{\nu} & =-\frac{2 T}{q_{0}}\left(1-\frac{q_{\|}^{2}}{q^{2}}\right) \operatorname{Im}\left(\frac{1}{Q^{2}-\Pi_{T, e}^{R}(Q)}-\frac{1}{Q^{2}-\Pi_{L, e}^{R}(Q)}\right),
\end{aligned}
$$

with

$$
\begin{aligned}
& \Pi_{L, e}^{R}(Q)=-\frac{Q^{2}}{q^{2}} M_{e}^{2}\left[1-\frac{q_{0}}{2 q} \ln \left(\frac{q_{0}+q}{q_{0}-q}\right)\right], \\
& \Pi_{T, e}^{R}(Q)=\frac{M_{e}^{2}}{2}\left[\left(\frac{q_{0}}{q}\right)^{2}-\frac{Q^{2}}{q^{2}} \frac{q_{0}}{2 q} \ln \left(\frac{q_{0}+q}{q_{0}-q}\right)\right] .
\end{aligned}
$$

Following the case with $Q^{a}=0$, we define

$$
f_{e}^{\mu}(\mathbf{k}, p)=-4 k_{\|}\left(k_{\|}+p\right) \int \frac{d k^{0}}{2 \pi} F_{e}(K, P) D_{e}^{\mu}(K, P) .
$$

Similarly,

$$
\left(\Gamma_{e}+i \delta E_{e}\right) f_{e}^{\mu}(\mathbf{k}, p)=(2 K+P)^{\mu}+\int \frac{d^{3} q}{(2 \pi)^{3}} C_{e}(\mathbf{q}, p) f_{e}^{\mu}(\mathbf{k}+\mathbf{q}, p),
$$

where

$$
C_{e}(\mathbf{q}, p)=\frac{g^{2}}{2} \int \frac{d q^{0}}{2 \pi} 2 \pi \delta\left(q^{0}-q_{\|}\right)\left(-i G_{\mu \nu, e}^{R R}(Q) \widehat{P}^{\mu} \widehat{P}^{\nu}\right) .
$$

The term proportional to $\Gamma_{e}$ can be written in terms of $C_{e}$,

$$
\Gamma_{e}=\frac{g^{2}}{2} \int \frac{d^{3} q d q_{0}}{(2 \pi)^{4}} 2 \pi \delta\left(q_{0}-q_{\|}\right)\left(-i G_{\mu \nu, e}^{R R}(Q) \widehat{P}^{\mu} \widehat{P}^{\nu}\right)
$$

Physically, this is because quark damping is due to scattering off of soft and diagonal gluons. We note that, by using $q \sim g T$ and $G_{\mu \nu, e}^{R R} \sim T / q^{3}, \Gamma_{e}$ is of order $g^{2} T$. This is suppressed by $1 / N$ compared with $\Gamma \sim g^{2} N T$, which is the damping rate when $Q^{a}=0$. The diagrams of quark damping are the same as gluon rung $M$, as illustrated in figure 13. Note that the Bose-Einstein enhancement fixes the color indices as $f=e$.

Therefore we have

$$
i \delta E_{e} f_{e}^{\mu}(\mathbf{k}, p)=(2 K+P)^{\mu}+\int \frac{d^{3} q}{(2 \pi)^{3}} C_{e}(\mathbf{q}, p)\left[f_{e}^{\mu}(\mathbf{k}+\mathbf{q}, p)-f_{e}^{\mu}(\mathbf{k}, p)\right] .
$$

We again need only an equation for projected $\mathbf{f}_{\mathbf{e}}$,

$$
i \delta E_{e} \mathbf{f}_{\mathbf{e} \perp}(\mathbf{k}, p)=2 \mathbf{k}_{\perp}+\int \frac{d^{3} q}{(2 \pi)^{3}} C_{e}(\mathbf{q}, p)\left[\mathbf{f}_{\mathbf{e} \perp}(\mathbf{k}+\mathbf{q}, p)-\mathbf{f}_{\mathbf{e} \perp}(\mathbf{k}, p)\right] .
$$




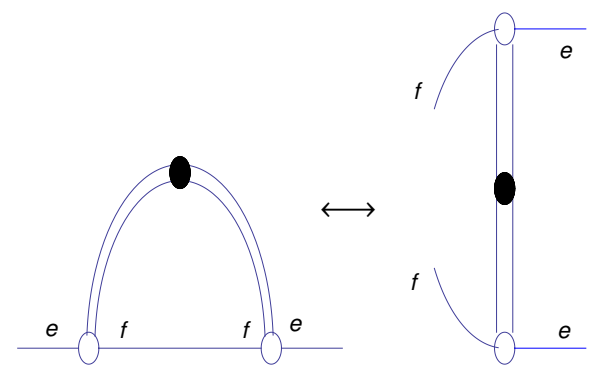

Figure 13. The correspondence between the diagrams for quark damping and for soft gluon exchange. The left-hand side is the quark self-energy, whose imaginary part gives the damping rate of the quark. The right-hand side is the diagram from gluon exchange, which is the cut diagram on the left-hand side. The double line notation is used.

The last changes are for eqs. (5.4) and (5.7). Following the derivation of ref. [107], eq. (5.4) becomes

$$
\begin{aligned}
G_{1122}= & \alpha_{1} G_{A A R R}+\alpha_{2} G_{A A A R}+\alpha_{3} G_{A A R A}+\alpha_{4} G_{A R A A} \\
& +\alpha_{5} G_{R A A A}+\alpha_{6} G_{A R R A}+\alpha_{7} G_{A R A R} \\
& +\beta_{1} G_{A A R R}^{\Delta}+\beta_{2} G_{A A A R}^{\Delta}+\beta_{3} G_{A A R A}^{\Delta}+\beta_{4} G_{A R A A}^{\Delta} \\
& +\beta_{5} G_{R A A A}^{\Delta}+\beta_{6} G_{A R R A}^{\Delta}+\beta_{7} G_{A R A R}^{\Delta}
\end{aligned}
$$

where $\Delta$ is defined as complex conjugation together with charge conjugation, i.e. flipping the sign of background color charge. The relevant coefficients are

$$
\alpha_{1} \simeq \beta_{1} \simeq \widetilde{n}_{e}\left(k_{\|}+p\right)\left(1-\widetilde{n}_{e}\left(k_{\|}\right)\right)
$$

As a result, eq. (5.7) becomes

$$
G_{1122}=\alpha_{1} G_{A A R R}\left(Q_{a}\right)+\alpha_{1} G_{A A R R}^{*}\left(-Q_{a}\right) .
$$

Note that the background charge enters the integral equation eq. (5.66) only through eqs. (5.43) and (5.54), which are independent of the sign of $Q_{a}$, as $\mathcal{A}$ is an even function. We still have

$$
G_{1122}=2 \alpha_{1} \operatorname{Re} G_{A A R R}\left(Q_{a}\right) .
$$

Finally, the collinear rate is given by

$$
p \frac{d \Gamma_{\gamma}}{d^{3} p}=\frac{\alpha_{\mathrm{em}} \sum_{f} q_{f}^{2}}{4 \pi^{2}} \int \frac{d k_{\|}}{2 \pi} \sum_{e=1}^{N} A_{e}\left(k_{\|}, p\right) \int \frac{d^{2} k_{\perp}}{(2 \pi)^{2}} \operatorname{Re}\left[2 \mathbf{k}_{\perp} \cdot \mathbf{f}_{\mathbf{e}_{\perp}}(k, p)\right],
$$

where

$$
A_{e}\left(k_{\|}, p\right)=\widetilde{n}_{e}\left(k_{\|}+p\right)\left(1-\widetilde{n}_{e}\left(k_{\|}\right)\right)\left(\frac{k_{\|}^{2}+\left(k_{\|}+p\right)^{2}}{2 k_{\|}^{2}\left(k_{\|}+p\right)^{2}}\right) .
$$

Note that the factor $N$ in eq. (5.42) is replaced by a sum over color index $e$ in eq. (5.71).

To summarize, the collinear rate in the presence of nontrivial Polyakov loop is given by eq. (5.71), with $\mathbf{f}_{\mathbf{e}_{\perp}}(k, p)$ the solution of eq. (5.66). All quantities which depend upon the 
background charge are defined in eqs. (5.43), (5.54), (5.58), (5.59), and (5.63). We note that the Polyakov loop effect enters separately in both the longitudinal and the transverse parts, as can be seen from eqs. (5.59), (5.71) and (5.72). In the longitudinal part, the Polyakov loop dependence is reflected in the distribution function factor $\widetilde{n}_{e}\left(k_{\|}+p\right)\left(1-\widetilde{n}_{e}\left(k_{\|}\right)\right)$. In the transverse part, the Polyakov loop effect appears in the asymptotic quark thermal mass $m_{e}$ and the gluon Debye mass $M_{e}$.

\subsection{Photon rate in the collinear regime at large $\mathrm{N}$}

To obtain the photon rate in collinear regime, we need to solve eq. (5.66). In the limit of large $N$, the collision term in eq. (5.66) is suppressed by $1 / N$. This can be understood as follows. Since $\delta E_{e} \sim m_{e}^{2} / k_{\|} \sim g^{2} T^{2} N / k_{\|}$, the left-hand side of eq. (5.66) is of order $g^{2} T^{2} N / k_{\|} \mathbf{f}_{\mathbf{e} \perp}$. On the other hand, by using eq. (5.64) the terms containing $C_{e}$ in the righthand side are of order $\Gamma_{e} \mathbf{f}_{\mathbf{e} \perp} \sim g^{2} T \mathbf{f}_{\mathrm{e} \perp}$. Thus, at sufficiently large $N$, terms in the latter are small compared to the former.

This allows us to solve eq. (5.66) perturbatively. The solutions to zeroth and first order in terms of $C_{e}$ are easily obtained. In the argument of all quantities, we suppress $p$ but indicate $\mathbf{k}$ :

$$
\begin{aligned}
\mathbf{f}_{\mathbf{e} \perp}{ }^{(0)} & =\frac{2 \mathbf{k}_{\perp}}{i \delta E_{e}(\mathbf{k})} \\
\mathbf{f}_{\mathbf{e} \perp}{ }^{(1)} & =\frac{1}{i \delta E_{e}(\mathbf{k})} \int \frac{d^{3} q}{(2 \pi)^{3}} C_{e}(q)\left[\frac{2\left(\mathbf{k}_{\perp}+\mathbf{q}_{\perp}\right)}{i \delta E_{e}(\mathbf{k}+\mathbf{q})}-\frac{2 \mathbf{k}_{\perp}}{i \delta E_{e}(\mathbf{k})}\right] .
\end{aligned}
$$

Only the solution to first order contributes to the photon rate. The relevant combination is

$$
\int \frac{d^{2} k_{\perp}}{(2 \pi)^{2}} \operatorname{Re}\left[2 \mathbf{k}_{\perp} \cdot \mathbf{f}_{\mathbf{e} \perp}(\mathbf{k})\right]=4 \int \frac{d^{2} k_{\perp}}{(2 \pi)^{2}} \int \frac{d^{3} q}{(2 \pi)^{3}} C_{e}(q)\left[\frac{k_{\perp}^{2}}{\delta E_{e}(\mathbf{k})^{2}}-\frac{\mathbf{k}_{\perp} \cdot\left(\mathbf{k}_{\perp}+\mathbf{q}_{\perp}\right)}{\delta E_{e}(\mathbf{k}) \delta E_{e}(\mathbf{k}+\mathbf{q})}\right] .
$$

We note that, because of this truncation, the LPM effect is suppressed. Using the sum rules of refs. [8, 108],

$$
\int \frac{d q^{0} d q^{\|}}{2 \pi} \delta\left(q^{0}-q^{\|}\right)\left(-i G_{\mu \nu, e}^{R R}(Q) \widehat{P}^{\mu} \widehat{P}^{\nu}\right)=T\left(\frac{1}{q_{\perp}^{2}}-\frac{1}{q_{\perp}^{2}+M_{e}^{2}}\right),
$$

eq. (5.74) simplifies to

$$
\begin{aligned}
\int \frac{d^{2} k_{\perp}}{(2 \pi)^{2}} \operatorname{Re}\left[2 \mathbf{k}_{\perp} \cdot \mathbf{f}_{\mathbf{e} \perp}(\mathbf{k})\right]= & 2 g^{2}\left(\frac{2 k_{\|}\left(k_{\|}+p\right)}{p}\right)^{2} \int \frac{d^{2} k_{\perp}}{(2 \pi)^{2}} \int \frac{d^{2} q_{\perp}}{(2 \pi)^{2}} \frac{T M_{e}^{2}}{q_{\perp}^{2}\left(q_{\perp}^{2}+M_{e}^{2}\right)} \\
& \times \frac{1}{k_{\perp}^{2}+m_{e}^{2}}\left(\frac{k_{\perp}^{2}}{k_{\perp}^{2}+m_{e}^{2}}-\frac{\mathbf{k}_{\perp} \cdot\left(\mathbf{k}_{\perp}+\mathbf{q}_{\perp}\right)}{|\mathbf{k}+\mathbf{q}|^{2}+m_{e}^{2}}\right) \\
= & -\frac{2 g^{2} T}{(2 \pi)^{2}}\left(\frac{k_{\|}\left(k_{\|}+p\right)}{p}\right)^{2} \int d k_{\perp}^{2} \int d q_{\perp}^{2} \frac{M_{e}^{2}}{q_{\perp}^{2}\left(q_{\perp}^{2}+M_{e}^{2}\right)} \\
& \times \frac{1}{k_{\perp}^{2}+m_{e}^{2}}\left(\frac{m_{e}^{2}}{k_{\perp}^{2}+m_{e}^{2}}-\frac{q_{\perp}^{2}+2 m_{e}^{2}}{2 \sqrt{\left(k_{\perp}^{2}+q_{\perp}^{2}+m_{e}^{2}\right)^{2}-\left(2 k_{\perp} q_{\perp}\right)^{2}}}\right),
\end{aligned}
$$


where in the second line we have performed the two angular integrations, and used the formula [8],

$$
\int_{0}^{\infty} d k_{\perp}^{2}\left(\frac{1}{k_{\perp}^{2}+m_{e}^{2}}-\frac{1}{\sqrt{\left(k_{\perp}^{2}+q_{\perp}^{2}+m_{e}^{2}\right)^{2}-\left(2 k_{\perp} q_{\perp}\right)^{2}}}\right)=0 .
$$

Here $k_{\perp}$ and $q_{\perp}$ are of order $g T$. Nevertheless, since the integrand is convergent in both the infrared and the ultraviolet, we can extend the range of the integrations of $k_{\perp}$ and $q_{\perp}$ to $[0, \infty]$. The result can be expressed in terms of a dimensionless function of the mass ratio $M_{e} / m_{e}$ :

$$
\int \frac{d^{2} k_{\perp}}{(2 \pi)^{2}} \operatorname{Re}\left[2 \mathbf{k}_{\perp} \cdot \mathbf{f}_{\mathbf{e} \perp}(\mathbf{k})\right] \equiv 2 g^{2}\left(\frac{2 k_{\|}\left(k_{\|}+p\right)}{p}\right)^{2} T \mathcal{G}\left(\frac{M_{e}}{m_{e}}\right),
$$

where

$$
\begin{aligned}
\mathcal{G}\left(\frac{M_{e}}{m_{e}}\right) \equiv & -\frac{1}{(4 \pi)^{2}} \int_{0}^{\infty} d k_{\perp}^{2}\left[\frac{m_{e}^{2}}{\left(k_{\perp}^{2}+m_{e}^{2}\right)^{2}} \ln \left|\frac{m_{e}^{2} M_{e}^{2}}{\left(k_{\perp}^{2}+m_{e}^{2}\right)^{2}}\right|\right. \\
& \left.-\frac{M_{e}^{2}-2 m_{e}^{2}}{2 \sqrt{A}\left(k_{\perp}^{2}+m_{e}^{2}\right)} \ln \frac{M_{e}^{2}\left(m_{e}^{2}-k_{\perp}^{2}-M_{e}^{2}-\sqrt{A}\right)}{M_{e}^{2}\left(m_{e}^{2}-k_{\perp}^{2}-M_{e}^{2}\right)+A-\left(k_{\perp}^{2}+m_{e}^{2}\right) \sqrt{A}}\right]
\end{aligned}
$$

with $A \equiv\left(M_{e}^{2}\right)^{2}-2 M_{e}^{2}\left(m_{e}^{2}-k_{\perp}^{2}\right)+\left(k_{\perp}^{2}+m_{e}^{2}\right)^{2}$. In general, the left hand side of eq. (5.78) is a complicated function of $k_{\|}, p$ and $M_{e} / m_{e}$. However, when LPM effect can be ignored, it factorizes into the form of the right hand side of eq. (5.78). The function $\mathcal{G}\left(M_{e} / m_{e}\right)$ can be interpreted as the suppression factor for collinear rate, which can be determined numerically.

Consequently, the collinear rate can be expressed as

$$
p \frac{d \Gamma_{\gamma}}{d^{3} p}=\frac{\alpha_{\mathrm{em}} \sum_{f} q_{f}^{2}}{4 \pi^{2}} \sum_{e=1}^{N} \int \frac{d k_{\|}}{2 \pi} \widetilde{n}_{e}\left(k_{\|}+p\right)\left(1-\widetilde{n}_{e}\left(k_{\|}\right)\right) 4 g^{2} T \mathcal{G}\left(\frac{M_{e}}{m_{e}}\right)\left(\frac{k_{\|}^{2}+\left(k_{\|}+p\right)^{2}}{p^{2}}\right) .
$$

The final $k_{\|}$-integral can be done as follows:

$$
\begin{aligned}
& \int \frac{d k_{\|}}{2 \pi}\left(\frac{k_{\|}^{2}+\left(k_{\|}+p\right)^{2}}{p^{2}}\right) \widetilde{n}_{e}\left(k_{\|}+p\right)\left(1-\widetilde{n}_{e}\left(k_{\|}\right)\right) \\
& =\int_{-\infty+i Q_{e}}^{+\infty+i Q_{e}} \frac{d l}{2 \pi}\left(\frac{2 l^{2}+p^{2} / 2-2 Q_{e}^{2}-4 i l Q_{e}}{p^{2}}\right) \widetilde{n}\left(l+\frac{p}{2}\right) \widetilde{n}\left(-l+\frac{p}{2}\right),
\end{aligned}
$$

where $l=k_{\|}+p / 2+i Q_{e}$. The integrand is exponentially suppressed as $\operatorname{Re} l \rightarrow \pm \infty$, which allows us to shift the integration contour to the real axis. The following integration formulas are useful:

$$
\begin{aligned}
\int \frac{d l}{2 \pi} \widetilde{n}\left(l+\frac{p}{2}\right) \widetilde{n}\left(-l+\frac{p}{2}\right) & =\frac{1}{2 \pi} \frac{p}{e^{p / T}-1}, \\
\int \frac{d l}{2 \pi} l^{2} \widetilde{n}\left(l+\frac{p}{2}\right) \widetilde{n}\left(-l+\frac{p}{2}\right) & =\frac{1}{2 \pi} \frac{p\left(4 \pi^{2} T^{2}+p^{2}\right)}{12\left(e^{p / T}-1\right)} .
\end{aligned}
$$


They can be obtained by integrating $l \widetilde{n}\left(l+\frac{p}{2}\right) \widetilde{n}\left(-l+\frac{p}{2}\right)$ and $l^{3} \widetilde{n}\left(l+\frac{p}{2}\right) \widetilde{n}\left(-l+\frac{p}{2}\right)$ along the rectangular contour bounded by $-\infty, \infty, \infty+2 \pi T i$ and $-\infty+2 \pi T i$. Using eq. (5.82), we obtain the collinear rate from eq. (5.80)

$$
p \frac{d \Gamma_{\gamma}}{d^{3} p}=\frac{\alpha_{\mathrm{em}} \alpha_{s} \sum_{f} q_{f}^{2}}{\pi^{2}} 2 T \sum_{e=1}^{N} \mathcal{G}\left(\frac{M_{e}}{m_{e}}\right) \frac{2 \pi^{2} T^{2}+2 p^{2}-6 Q_{e}^{2}}{3 p\left(e^{p / T}-1\right)} .
$$

For a hard photon, where $p \gg T, Q_{e}$, the collinear rate is simplified to

$$
p \frac{d \Gamma_{\gamma}}{d^{3} p} \simeq \frac{\alpha_{\mathrm{em}} \alpha_{s} \sum_{f} q_{f}^{2}}{\pi^{2}} \frac{4 T p}{3} \sum_{e=1}^{N} \mathcal{G}\left(\frac{M_{e}}{m_{e}}\right) e^{-p / T},
$$

whose parametric behavior is a Boltzmann factor times a term linear in $p$. This $p$ dependence is consistent with AMY's analysis without the LPM mechanism, and the analysis at two-loop order [109].

Note that the Polyakov loop only enters through the sum $\sum_{e} \mathcal{G}\left(M_{e} / m_{e}\right)$. In figure 14 we show the temperature dependence of this function when $N=N_{f}=3$. While each individual term $\mathcal{G}\left(M_{e} / m_{e}\right)$ changes with temperature, especially near $T_{c}$, the sum is remarkably flat, with $\sum_{e} \mathcal{G}\left(M_{e} / m_{e}\right) \simeq 3 \times 0.015$ over a wide range of temperature.

From eq. (5.84) the collinear rate is not suppressed in the confined phase. At first this is a surprising result, and it is worth discussing in some detail. It happens because the soft gluon which is radiated is diagonal in color space, so the quarks in the initial and final state have the same color indices. The distribution factor which appears in eq. (5.80) is $\widetilde{n}_{e}\left(k_{\|}+p\right)\left(1-\widetilde{n}_{e}\left(k_{\|}\right)\right)=n(p)\left(\widetilde{n}_{e}\left(k_{\|}\right)-\widetilde{n}_{e}\left(k_{\|}+p\right)\right)$. For large $p>0$, this factor is nonzero only when $k_{\|}+p$ is positive, and $k_{\|}$is negative [11, 109]. This corresponds to pair annihilation, as illustrated in figure 15; the other processes correspond to bremsstrahlung, and do not contribute in this limit.

Since $k_{\|}+p$ is positive, $k_{\|}$is not only negative, but large. Consequently, as $p \gg T$, we can use a Boltzmann approximation for the statistical distribution functions:

$$
\begin{aligned}
\frac{1}{N} \sum_{e=1}^{N} \widetilde{n}_{e}\left(k_{\|}+p\right)\left(1-\widetilde{n}_{e}\left(k_{\|}\right)\right) & =\frac{1}{N} \sum_{e=1}^{N} \widetilde{n}_{e}\left(k_{\|}+p\right) \widetilde{n}_{\bar{e}}\left(-k_{\|}\right) \\
& \simeq \frac{1}{N} \sum_{e=1}^{N} e^{-\left(k_{\|}+p-i Q_{e}\right) / T} e^{-\left(-k_{\|}+i Q_{e}\right) / T} \\
& =e^{-p / T} .
\end{aligned}
$$

Thus the collinear contribution is not suppressed in the confined phase because the phases cancel between the quark and anti-quark. This is exactly the same cancellation as found for dilepton production, and rather unlike the color flow for the contribution to photon production from $2 \rightarrow 2$ scattering.

This completes our derivation of photon rate in the semi-QGP, with a nontrivial Polyakov loop at large $N$. The result is a sum of leading logarithmic term from the rate for $2 \leftrightarrow 2$, eq. (4.71), and the collinear rate in the large $N$ limit, eq. (5.83). We emphasize that rates for $2 \leftrightarrow 2$ and collinear emission depend upon the Polyakov loop in completely 


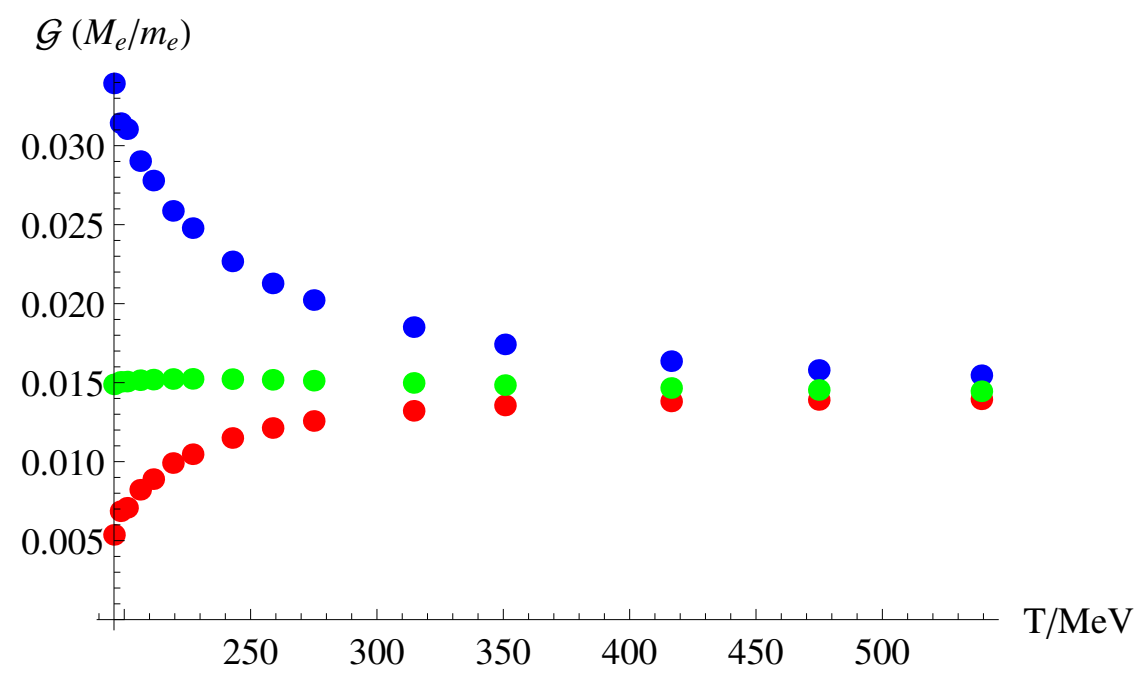

Figure 14. Temperature dependence of function $\mathcal{G}\left(M_{e} / m_{e}\right)$ in eq. (5.84), which is the suppression factor for collinear rate due to background color charge when LPM effect can be ignored. In $N=3$, the background color charge is parametrized as $Q_{e}=(-Q, 0,+Q)$. Points of different colors in the figure correspond to $e=1$ (red), $e=2$ (blue) and $\mathcal{G}$ averaged over three colors (green).

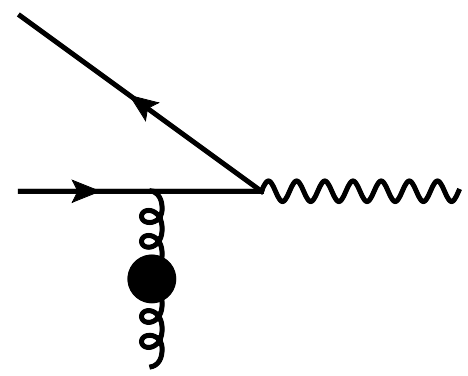

Figure 15. The collinear pair annihilation process. The curly line with a blob is the gluon HTLresummed propagator.

different ways. When the Polyakov loop is small, the rate for $2 \leftrightarrow 2$ is suppressed while that for collinear emission is not. We note that our results are valid only for small values of $g^{2}$ and large $N$. At moderate values of the coupling constant, corrections due to the constant under the logarithm become important. At moderate values of $N$, the LPM effect becomes relevant, and will produce cancellations between diagrams with different number of loops, suppressing the photon rate in the collinear regime.

\subsection{Why the LPM effect is suppressed in the semi-QGP}

A nontrivial Polyakov loop is understood as from quantum fluctuations in $A_{0}$ of order $T / g$. This background gauge field affects quarks and gluons in different ways. As shown in ref. [85], it reduces the density of hard quarks. It also acts as a Higgs effect for gluons, giving mass of order $T$ to off-diagonal gluons, while leaving diagonal gluons massless. The only gluons which scatter off of quarks in the large $N$ limit are diagonal, and so are reduced by $1 / N$. 
In the perturbative QGP, the LPM effect is relevant because the photon formation time, $t_{F}$, is comparable to the mean free path, $\lambda$, of a quark undergoing multiple scattering with gluons in the medium. The formation time is the time scale when a collinear photon can be well separated from the quark, which is

$$
t_{F} \sim \frac{1}{\delta E} \sim \frac{T}{k_{\perp}^{2}+m^{2}} \sim \frac{1}{g^{2} N T} .
$$

On the other hand, the mean free path has the same order of magnitude as the damping rate of a quark in the thermal bath, with $\lambda \sim 1 / \Gamma \sim 1 /\left(g^{2} N T\right)$, which is comparable to $t_{F}$.

A nontrivial Polyakov loop modifies the two scales differently. The thermal mass $m$ results from interactions of a quark with hard thermal gluons. The Polyakov loop suppresses the quark and the gluon density, and thus also $m$, by a loop dependent factor. The damping rate is due to the scattering off of soft gluons, but as these are suppressed for $Q^{a} \sim T$, only the scattering off of soft, diagonal gluons matters. Consequently, $\lambda \sim 1 /\left(g^{2} T\right)$ times a loop dependent factor, so at large $N, \lambda \gg t_{F}$. This implies that quarks rarely scatter more than once during the emission of a photon, and thus the LPM effect can be ignored.

\section{Summary and concluding remarks}

We calculated the production rates of the dilepton and the real photon in a matrix model of the semi-QGP. The main results of this paper are eqs. (3.13), (4.71), and (5.84). The dilepton production rate was found to be slightly enhanced in the confined phase due to a cancellation in the phases of the statistical distribution functions for the quark and antiquark [98]. By contrast, the photon production rate due to the $2 \rightarrow 2$ scattering is strongly suppressed for small values of the Polyakov loop, as the phases in the distribution functions do not cancel. We showed that the collinear contribution to the photon production is suppressed at large $N$ in the semi-QGP, since when the Polyakov loop is small, the $Q^{a}$ 's are large, and off-diagonal gluons do not experience Bose-Einstein enhancement. We computed the collinear contribution at large $N$, and found that because of a cancellation of phases, like dilepton production it is not suppressed even in the confined phase.

These results will modify the theoretical predictions for thermal production in heavy ion collisions. Certainly the production rates for dileptons and photons are altered. The elliptic flow for these particles are similarly modified, as the total elliptic flow is an average over all the phases, from the initial state, to the QGP, to hadrons. These effects were previously discussed in ref. [98]. However, in that work the modifications of photon production from $2 \rightarrow 2$ scattering and from collinear emission were not considered separately. Clearly a more realistic treatment is called for.

In the current analysis, the effect of the confinement is taken into account as a nontrivial value of the Polyakov loop. It is also interesting to consider the effect of the chiral symmetry restoration as well as confinement [99, 105].

For the future, besides doing a more complete analysis of photon production, the most urgent problem is to compute radiative energy loss for light quarks. This is closely related to collinear photon emission, and so we expect that near $T_{c}$, it will be dominated by diagonal gluons for processes in which the color phases cancel. 


\section{Acknowledgments}

R.D.P. would like to thank C. Islam, S. Majumder, N. Haque, and M. Mustafa for discussions about their work on dilepton production in the PNJL model [99] in Mumbai, at the workshop "QCD at high density", ${ }^{1}$ and in Kolkata, at the "7th International Conference on Physics and Astrophysics of the Quark-Gluon Plasma". ${ }^{2}$ S.L. would like to thank B. Wu and L. Yaffe for useful discussions. Y.H. is supported by JSPS KAKENHI (Grants No. 24740184), and by the RIKEN iTHES Project. S.L. is supported by the RIKEN Foreign Postdoctoral Researchers Program. R.D.P. is supported by DOE Contract DE-SC0012704 and by the RIKEN/BNL Research Center. D.S. is supported by JSPS Strategic Young Researcher Overseas Visits Program for Accelerating Brain Circulation (No. R2411).

\section{A Corrections to Boltzmann approximation to thermal distribution functions}

We will argue that correction to (4.11) is suppressed by additional exponential. We illustrate this in case of Compton scattering. The exact thermal distribution factors can be expressed as

$$
\begin{aligned}
& \frac{1}{e^{\beta E_{1}}+1} \frac{1}{e^{\beta E_{2}}-1}\left(1-\frac{1}{e^{\beta E^{\prime}}+1}\right) \\
& =\sum_{m, n=0}^{\infty}(-1)^{m} e^{-(m+n+2) \beta x / 2-(m-n) \beta y / 2}\left(1-\frac{1}{e^{\beta(x-E)}+1}\right) .
\end{aligned}
$$

Now the $y$-integral becomes

$$
\int \frac{d y e^{-(m-n) \beta y / 2}}{\sqrt{a y^{2}+b y+c}}=\frac{\pi}{\sqrt{-a}} e^{-(m-n) \beta y_{0} / 2} I_{0}\left(\frac{m-n}{2} \beta \Delta y\right),
$$

where

$$
\begin{aligned}
y_{0} & =\frac{t-u}{t+u}(x-2 E), \\
\Delta y & =-\frac{2 \sqrt{t u(t+u+4 E(x-E))}}{t+u},
\end{aligned}
$$

and $I_{0}(z)$ is the modified Bessel function of the first kind. Note that the leading logarithmic contribution comes from $t \sim \mu^{2}, s \sim p T$, which implies $\Delta y \sim \mu$. Therefore we may set $I_{0}((m-n) \beta \Delta y / 2)=1$. This leads to the following $x$-integral

$$
\begin{aligned}
& \int_{p+\frac{s}{4 p}}^{\infty} d x e^{-(\beta+\delta) x}\left(1-\frac{1}{e^{\beta(x-p)}+1}\right) \\
& =\left.\frac{e^{-\beta p-\delta x}}{\delta} F\left(1,-\frac{\delta}{\beta}, 1-\frac{\delta}{\beta},-e^{\beta(x-p)}\right)\right|_{x=p+s /(4 p)} \\
& =\frac{e^{-(\beta+\delta)(p+s /(4 p))}}{\delta+\beta} F\left(1,1+\frac{\delta}{\beta}, 2+\frac{\delta}{\beta},-e^{-\beta s /(4 p)}\right),
\end{aligned}
$$

\footnotetext{
${ }^{1}$ http://theory.tifr.res.in/ qcd2015.

${ }^{2}$ http://indico.vecc.gov.in/indico/internalPage.py?pageId=37\&confId=29.
} 
with $\delta=\beta(m+n) / 2+\beta(m-n)(t-u) /(2(t+u))>0$ unless $m=n=0$. Here, $F(a, b, c, z)$ is the hypergeometric function. For non-vanishing $\delta$, there is an additional exponential suppression factor $e^{-\delta(p+s /(4 p))}$. Therefore, we conclude any terms with non-vanishing $m$ or $n$ is negligible in eq. (A.1), leaving only the term with $m=n=0$, which corresponds to the Boltzmann approximation.

\section{B Thermal gluon mass in the presence of Polyakov loop}

We regard $N_{f} \sim N$ as a large number. Naively, the $F$ and $G$ terms give the same order contribution in $N$ because $\delta_{a b} \sim 1 / N$, however, as we show below, the $G$ term is suppressed by $1 / N$ compared to the $F$ term. By plugging eq. (5.48) into eq. (5.47), we obtain the first few terms explicitly:

$$
\begin{aligned}
& \frac{1}{Q^{2}}\left(\delta_{a b}-\frac{1}{N}\right), \\
& \frac{1}{\left(Q^{2}\right)^{2}}\left(\delta_{a b} F_{a}-\frac{1}{N} L_{1}(F, G)\right), \\
& \frac{1}{\left(Q^{2}\right)^{3}}\left(\delta_{a b} F_{a}^{2}-\frac{1}{N} L_{2}(F, G)\right) .
\end{aligned}
$$

Here $L_{1}\left(L_{2}\right)$ are complicated functions linear(quadratic) in $F$ or $G$. By induction, we can obtain the form of propagator with $n$ self-energy insertions

$$
\frac{1}{\left(Q^{2}\right)^{n+1}}\left(\delta_{a b} F_{a}^{n}-\frac{1}{N} L_{n}(F, G)\right) .
$$

It is easy to see the $\delta_{a b}$ term can be summed as a geometric series, while the $1 / N$ term is not summable in simple manner. In any case, the resummed gluon propagator has the following color structure

$$
\delta_{a b} A_{a}(Q)-\frac{1}{N} B_{a b}(Q)
$$

with $A_{a}(Q)$ and $B_{a b}(Q)$ of the same order in $N$. Note $A_{a}(Q)=\left(Q^{2}-F_{a}\right)^{-1} \delta_{a b}$ is entirely from $F$, while $B_{a b}(Q)$ has contribution from both $F$ and $G$.

Now we insert the resummed propagator into the graphical element $M$. Focusing again on the color structure, we obtain after summing over gluon color indices:

$$
\begin{aligned}
& \left(T^{a a}\right)_{e f}\left(T^{b b}\right)_{h g}\left[\delta_{a b} A_{a}-\frac{1}{N} B_{a b}\right] \\
& =\delta_{e f} \delta_{h g} \frac{1}{2}\left[\delta_{f g} A_{f}-\frac{1}{N}\left(A_{f}+A_{g}\right)+\frac{1}{N^{2}} \sum_{c=1}^{N} A_{c}-\frac{1}{N} B_{f g}\right. \\
& \left.+\frac{1}{N^{2}} \sum_{c=1}^{N}\left(B_{f c}+B_{c g}\right)-\frac{1}{N^{3}} \sum_{c, d=1}^{N} B_{c d}\right]
\end{aligned}
$$

In the above, we have suppressed the $P$ dependence of $A$ and $B$ for notational simplicity. Formally all terms are of the same order if we regard $\delta_{f g} \sim 1 / N$ and sum as $\sim N$. However, 
we have learned from the case without background color charge that the structure of $M$ is ultimately contracted with $\delta_{e h}$ on the left, which brings eq. (B.4) into the following form

$$
\delta_{f g}\left[\delta_{f g} A_{f}-\frac{1}{N}\left(A_{f}+A_{g}\right)+\frac{1}{N^{2}} \sum_{c=1}^{N} A_{c}-\frac{1}{N} B_{f g}+\frac{1}{N^{2}} \sum_{c=1}^{N}\left(B_{f c}+B_{c g}\right)-\frac{1}{N^{3}} \sum_{c, d=1}^{N} B_{c d}\right] .
$$

We see the $\delta_{f g}$ in the first term of the bracket becomes redundant. We can replace it by 1 . Consequently, all other terms are suppressed by $1 / N$. We will keep only the first term, which is fortunately easy to calculate. This approximation amounts to dropping the $1 / N$ term in eq. (5.51), leading to the gluon Debye mass, eq. (5.54).

Open Access. This article is distributed under the terms of the Creative Commons Attribution License (CC-BY 4.0), which permits any use, distribution and reproduction in any medium, provided the original author(s) and source are credited.

\section{References}

[1] U.W. Heinz, Early Collective Expansion: Relativistic Hydrodynamics and the Transport Properties of QCD Matter, Landolt-Börnstein 23 (2010) 240 [arXiv:0901.4355].

[2] U. Heinz and R. Snellings, Collective flow and viscosity in relativistic heavy-ion collisions, Ann. Rev. Nucl. Part. Sci. 63 (2013) 123 [arXiv:1301.2826] [INSPIRE].

[3] C. Gale, S. Jeon and B. Schenke, Hydrodynamic Modeling of Heavy-Ion Collisions, Int. J. Mod. Phys. A 28 (2013) 1340011 [arXiv:1301.5893] [InSPIRE].

[4] B. Schenke, S. Jeon and C. Gale, $(3+1) D$ hydrodynamic simulation of relativistic heavy-ion collisions, Phys. Rev. C 82 (2010) 014903 [arXiv:1004.1408] [INSPIRE].

[5] B. Schenke, S. Jeon and C. Gale, Elliptic and triangular flow in event-by-event $(3+1) D$ viscous hydrodynamics, Phys. Rev. Lett. 106 (2011) 042301 [arXiv: 1009.3244] [INSPIRE].

[6] R. Baier, H. Nakkagawa, A. Niegawa and K. Redlich, Production rate of hard thermal photons and screening of quark mass singularity, Z. Phys. C 53 (1992) 433 [InSPIRE].

[7] J.I. Kapusta, P. Lichard and D. Seibert, High-energy photons from quark-gluon plasma versus hot hadronic gas, Phys. Rev. D 44 (1991) 2774 [Erratum ibid. D 47 (1993) 4171] [INSPIRE].

[8] P. Aurenche, F. Gelis and H. Zaraket, Landau-Pomeranchuk-Migdal effect in thermal field theory, Phys. Rev. D 62 (2000) 096012 [hep-ph/0003326] [inSPIRE].

[9] P.B. Arnold, G.D. Moore and L.G. Yaffe, Photon and gluon emission in relativistic plasmas, JHEP 06 (2002) 030 [hep-ph/0204343] [INSPIRE].

[10] P.B. Arnold, G.D. Moore and L.G. Yaffe, Photon emission from ultrarelativistic plasmas, JHEP 11 (2001) 057 [hep-ph/0109064] [InSPIRE].

[11] P.B. Arnold, G.D. Moore and L.G. Yaffe, Photon emission from quark gluon plasma: Complete leading order results, JHEP 12 (2001) 009 [hep-ph/0111107] [INSPIRE].

[12] J. Ghiglieri, J. Hong, A. Kurkela, E. Lu, G.D. Moore and D. Teaney, Next-to-leading order thermal photon production in a weakly coupled quark-gluon plasma, JHEP 05 (2013) 010 [arXiv:1302.5970] [INSPIRE]. 
[13] J. Ghiglieri and G.D. Moore, Low Mass Thermal Dilepton Production at NLO in a Weakly Coupled quark-gluon Plasma, JHEP 12 (2014) 029 [arXiv:1410.4203] [INSPIRE].

[14] C.H. Lee, J. Wirstam, I. Zahed and T.H. Hansson, Thermal dileptons from a nonperturbative quark-gluon phase, Phys. Lett. B 448 (1999) 168 [hep-ph/9809440] [INSPIRE].

[15] K. Dusling, D. Teaney and I. Zahed, Thermal dimuon yields at NA60, Phys. Rev. C 75 (2007) 024908 [nucl-th/0604071] [INSPIRE].

[16] K. Dusling and I. Zahed, Low mass dilepton radiation at RHIC, Nucl. Phys. A 825 (2009) 212 [arXiv:0712.1982] [INSPIRE].

[17] K. Dusling and I. Zahed, Thermal photons from heavy ion collisions: A spectral function approach, Phys. Rev. C 82 (2010) 054909 [arXiv:0911.2426] [INSPIRE].

[18] J. Manninen, E.L. Bratkovskaya, W. Cassing and O. Linnyk, Dilepton production in $p+p$, $C u+C u$ and $A u+A u$ collisions at 200 AGeV, Eur. Phys. J. C 71 (2011) 1615 [arXiv: 1005.0500] [INSPIRE].

[19] P. Staig and E. Shuryak, Production of soft $e^{+} e^{-}$Pairs in Heavy Ion Collisions at RHIC by Semi-coherent Two Photon Processes, arXiv:1005.3531 [INSPIRE].

[20] O. Linnyk, W. Cassing, J. Manninen, E.L. Bratkovskaya and C.M. Ko, Analysis of dilepton production in $A u+A u$ collisions at $\sqrt{s_{N N}}=200 \mathrm{GeV}$ within the Parton-Hadron-String Dynamics (PHSD) transport approach, Phys. Rev. C 85 (2012) 024910 [arXiv:1111.2975] [INSPIRE].

[21] O. Linnyk et al., Dilepton production in proton-proton and $\mathrm{Pb}+\mathrm{Pb}$ collisions at $\sqrt{s_{N N}}=2.76 \mathrm{TeV}$, Phys. Rev. C 87 (2013) 014905 [arXiv:1208.1279] [InSPIRE].

[22] P.M. Hohler and R. Rapp, Is $\rho$-Meson Melting Compatible with Chiral Restoration?, Phys. Lett. B 731 (2014) 103 [arXiv: 1311.2921] [INSPIRE].

[23] C.-H. Lee and I. Zahed, Electromagnetic Radiation in Hot QCD Matter: Rates, Electric Conductivity, Flavor Susceptibility and Diffusion, Phys. Rev. C 90 (2014) 025204 [arXiv: 1403.1632] [INSPIRE].

[24] R. Rapp, Dilepton Spectroscopy of QCD Matter at Collider Energies, Adv. High Energy Phys. 2013 (2013) 148253 [arXiv: 1304.2309] [InSPIRE].

[25] G. Vujanovic, C. Young, B. Schenke, R. Rapp, S. Jeon and C. Gale, Dilepton emission in high-energy heavy-ion collisions with viscous hydrodynamics, Phys. Rev. C 89 (2014) 034904 [arXiv: 1312.0676] [INSPIRE].

[26] R. Chatterjee, E.S. Frodermann, U.W. Heinz and D.K. Srivastava, Elliptic flow of thermal photons in relativistic nuclear collisions, Phys. Rev. Lett. 96 (2006) 202302 [nucl-th/0511079] [INSPIRE].

[27] E.L. Bratkovskaya, S.M. Kiselev and G.B. Sharkov, Direct photon production from hadronic sources in high-energy heavy-ion collisions, Phys. Rev. C 78 (2008) 034905 [arXiv: 0806.3465] [INSPIRE].

[28] H. van Hees, C. Gale and R. Rapp, Thermal Photons and Collective Flow at the Relativistic Heavy-Ion Collider, Phys. Rev. C 84 (2011) 054906 [arXiv:1108.2131] [InSPIRE].

[29] G. Basar, D. Kharzeev, D. Kharzeev and V. Skokov, Conformal anomaly as a source of soft photons in heavy ion collisions, Phys. Rev. Lett. 109 (2012) 202303 [arXiv:1206.1334] [INSPIRE]. 
[30] A. Bzdak and V. Skokov, Anisotropy of photon production: initial eccentricity or magnetic field, Phys. Rev. Lett. 110 (2013) 192301 [arXiv:1208.5502] [INSPIRE].

[31] K. Fukushima and K. Mameda, Wess-Zumino-Witten action and photons from the Chiral Magnetic Effect, Phys. Rev. D 86 (2012) 071501 [arXiv:1206.3128] [INSPIRE].

[32] F.-M. Liu and S.-X. Liu, quark-gluon plasma formation time and direct photons from heavy ion collisions, Phys. Rev. C 89 (2014) 034906 [arXiv:1212.6587] [InSPIRE].

[33] C. Shen, U.W. Heinz, J.-F. Paquet, I. Kozlov and C. Gale, Anisotropic flow of thermal photons as a quark-gluon plasma viscometer, Phys. Rev. C 91 (2015) 024908 [arXiv:1308.2111] [INSPIRE].

[34] C. Shen, U.W. Heinz, J.-F. Paquet and C. Gale, Thermal photons as a quark-gluon plasma thermometer reexamined, Phys. Rev. C 89 (2014) 044910 [arXiv: 1308. 2440] [INSPIRE].

[35] O. Linnyk, V.P. Konchakovski, W. Cassing and E.L. Bratkovskaya, Photon elliptic flow in relativistic heavy-ion collisions: hadronic versus partonic sources, Phys. Rev. C $8 \mathbf{8}$ (2013) 034904 [arXiv: 1304.7030] [INSPIRE].

[36] O. Linnyk, W. Cassing and E.L. Bratkovskaya, Centrality dependence of the direct photon yield and elliptic flow in heavy-ion collisions at $\sqrt{s_{N N}}=200 \mathrm{GeV}$, Phys. Rev. C 89 (2014) 034908 [arXiv: 1311.0279] [INSPIRE].

[37] B. Müller, S.-Y. Wu and D.-L. Yang, Elliptic flow from thermal photons with magnetic field in holography, Phys. Rev. D 89 (2014) 026013 [arXiv:1308.6568] [INSPIRE].

[38] K.A. Mamo, Enhanced thermal photon and dilepton production in strongly coupled $N=4$ SYM plasma in strong magnetic field, JHEP 08 (2013) 083 [arXiv:1210.7428] [INSPIRE].

[39] G. Basar, D.E. Kharzeev and E.V. Shuryak, Magneto-sonoluminescence and its signatures in photon and dilepton production in relativistic heavy ion collisions, Phys. Rev. C 90 (2014) 014905 [arXiv: 1402.2286] [INSPIRE].

[40] H. van Hees, M. He and R. Rapp, Pseudo-Critical Enhancement of Thermal Photons in Relativistic Heavy-Ion Collisions, Nucl. Phys. A 933 (2015) 256 [arXiv:1404.2846] [INSPIRE].

[41] L. McLerran and B. Schenke, The Glasma, Photons and the Implications of Anisotropy, Nucl. Phys. A 929 (2014) 71 [arXiv:1403.7462] [INSPIRE].

[42] A. Monnai, Thermal photon $v_{2}$ with slow quark chemical equilibration, Phys. Rev. C 90 (2014) 021901 [arXiv: 1403.4225] [INSPIRE].

[43] M. Dion, J.-F. Paquet, B. Schenke, C. Young, S. Jeon and C. Gale, Viscous photons in relativistic heavy ion collisions, Phys. Rev. C 84 (2011) 064901 [arXiv:1109.4405] [INSPIRE].

[44] N. Haque, A. Bandyopadhyay, J.O. Andersen, M.G. Mustafa, M. Strickland and N. Su, Three-loop HTLpt thermodynamics at finite temperature and chemical potential, JHEP 05 (2014) 027 [arXiv : 1402.6907] [INSPIRE].

[45] P. Huovinen and P. Petreczky, QCD Equation of State and Hadron Resonance Gas, Nucl. Phys. A 837 (2010) 26 [arXiv:0912.2541] [inSPIRE].

[46] A. Andronic, P. Braun-Munzinger, J. Stachel and M. Winn, Interacting hadron resonance gas meets lattice QCD, Phys. Lett. B 718 (2012) 80 [arXiv:1201.0693] [INSPIRE]. 
[47] S. Caron-Huot, P. Kovtun, G.D. Moore, A. Starinets and L.G. Yaffe, Photon and dilepton production in supersymmetric Yang-Mills plasma, JHEP 12 (2006) 015 [hep-th/0607237] [INSPIRE].

[48] A. Parnachev and D.A. Sahakyan, Photoemission with Chemical Potential from QCD Gravity Dual, Nucl. Phys. B 768 (2007) 177 [hep-th/0610247] [InSPIRE].

[49] A. Nata Atmaja and K. Schalm, Photon and Dilepton Production in Soft Wall AdS/QCD, JHEP 08 (2010) 124 [arXiv:0802.1460] [INSPIRE].

[50] A. Peshier and W. Cassing, The hot non-perturbative gluon plasma is an almost ideal colored liquid, Phys. Rev. Lett. 94 (2005) 172301 [hep-ph/0502138] [INSPIRE].

[51] W. Cassing, QCD thermodynamics and confinement from a dynamical quasiparticle point of view, Nucl. Phys. A 791 (2007) 365 [arXiv:0704.1410] [InSPIRE].

[52] W. Cassing, Dynamical quasiparticles properties and effective interactions in the sQGP, Nucl. Phys. A 795 (2007) 70 [arXiv:0707.3033] [INSPIRE].

[53] W. Cassing and E.L. Bratkovskaya, Parton-Hadron-String Dynamics: an off-shell transport approach for relativistic energies, Nucl. Phys. A 831 (2009) 215 [arXiv:0907.5331] [INSPIRE].

[54] E.L. Bratkovskaya, W. Cassing, V.P. Konchakovski and O. Linnyk, Parton-Hadron-String Dynamics at Relativistic Collider Energies, Nucl. Phys. A 856 (2011) 162 [arXiv:1101.5793] [INSPIRE].

[55] V. Ozvenchuk, O. Linnyk, M.I. Gorenstein, E.L. Bratkovskaya and W. Cassing, Dynamical equilibration of strongly interacting "infinite" parton matter within the parton-hadron-string dynamics transport approach, Phys. Rev. C 87 (2013) 024901 [arXiv:1203.4734] [INSPIRE].

[56] K. Fukushima, Chiral effective model with the Polyakov loop, Phys. Lett. B 591 (2004) 277 [hep-ph/0310121] [INSPIRE].

[57] H. Hansen, W.M. Alberico, A. Beraudo, A. Molinari, M. Nardi and C. Ratti, Mesonic correlation functions at finite temperature and density in the Nambu-Jona-Lasinio model with a Polyakov loop, Phys. Rev. D 75 (2007) 065004 [hep-ph/0609116] [INSPIRE].

[58] S.K. Ghosh, A. Lahiri, S. Majumder, M.G. Mustafa, S. Raha and R. Ray, Quark Number Susceptibility: Revisited with Fluctuation-Dissipation Theorem in mean field theories, Phys. Rev. D 90 (2014) 054030 [arXiv: 1407.7203] [inSPIRE].

[59] J. Braun, H. Gies and J.M. Pawlowski, Quark Confinement from Color Confinement, Phys. Lett. B 684 (2010) 262 [arXiv:0708.2413] [INSPIRE].

[60] F. Marhauser and J.M. Pawlowski, Confinement in Polyakov Gauge, arXiv:0812.1144 [INSPIRE].

[61] J. Braun, L.M. Haas, F. Marhauser and J.M. Pawlowski, Phase Structure of Two-Flavor QCD at Finite Chemical Potential, Phys. Rev. Lett. 106 (2011) 022002 [arXiv:0908. 0008] [INSPIRE].

[62] T.K. Herbst, J.M. Pawlowski and B.-J. Schaefer, The phase structure of the Polyakov-quark-meson model beyond mean field, Phys. Lett. B 696 (2011) 58 [arXiv: 1008.0081] [INSPIRE].

[63] J. Braun, A. Eichhorn, H. Gies and J.M. Pawlowski, On the Nature of the Phase Transition in $\mathrm{SU}(N), \mathrm{Sp}(2)$ and $E_{7}$ Yang-Mills theory, Eur. Phys. J. C 70 (2010) 689 [arXiv: 1007.2619] [INSPIRE]. 
[64] L. Fister and J.M. Pawlowski, Confinement from Correlation Functions, Phys. Rev. D 88 (2013) 045010 [arXiv: 1301.4163] [INSPIRE].

[65] T.K. Herbst, J.M. Pawlowski and B.-J. Schaefer, Phase structure and thermodynamics of QCD, Phys. Rev. D 88 (2013) 014007 [arXiv:1302.1426] [InSPIRE].

[66] C.S. Fischer, L. Fister, J. Luecker and J.M. Pawlowski, Polyakov loop potential at finite density, Phys. Lett. B 732 (2014) 273 [arXiv:1306.6022] [INSPIRE].

[67] T.K. Herbst, M. Mitter, J.M. Pawlowski, B.-J. Schaefer and R. Stiele, Thermodynamics of QCD at vanishing density, Phys. Lett. B 731 (2014) 248 [arXiv:1308.3621] [InSPIRE].

[68] M. Haas, L. Fister and J.M. Pawlowski, Gluon spectral functions and transport coefficients in Yang-Mills theory, Phys. Rev. D 90 (2014) 091501 [arXiv: 1308.4960] [INSPIRE].

[69] M. Mitter, J.M. Pawlowski and N. Strodthoff, Chiral symmetry breaking in continuum QCD, Phys. Rev. D 91 (2015) 054035 [arXiv:1411.7978] [INSPIRE].

[70] J. Braun, L. Fister, J.M. Pawlowski and F. Rennecke, From Quarks and Gluons to Hadrons: Chiral Symmetry Breaking in Dynamical QCD, arXiv:1412.1045 [INSPIRE].

[71] U. Reinosa, J. Serreau, M. Tissier and N. Wschebor, Deconfinement transition in SU(N) theories from perturbation theory, Phys. Lett. B 742 (2015) 61 [arXiv:1407.6469] [INSPIRE].

[72] U. Reinosa, J. Serreau, M. Tissier and N. Wschebor, Deconfinement transition in $\mathrm{SU}(2)$ Yang-Mills theory: A two-loop study, Phys. Rev. D 91 (2015) 045035 [arXiv:1412.5672] [INSPIRE].

[73] U. Reinosa, J. Serreau and M. Tissier, Perturbative study of the QCD phase diagram for heavy quarks at nonzero chemical potential, Phys. Rev. D 92 (2015) 025021 [arXiv: 1504.02916] [INSPIRE].

[74] R.D. Pisarski, Effective Theory of Wilson Lines and Deconfinement, Phys. Rev. D 74 (2006) 121703 [hep-ph/0608242] [INSPIRE].

[75] Y. Hidaka and R.D. Pisarski, Suppression of the Shear Viscosity in a "semi" Quark Gluon Plasma, Phys. Rev. D 78 (2008) 071501 [arXiv:0803.0453] [InSPIRE].

[76] Y. Hidaka and R.D. Pisarski, Hard thermal loops, to quadratic order, in the background of a spatial 't Hooft loop, Phys. Rev. D 80 (2009) 036004 [arXiv:0906.1751] [InSPIRE].

[77] Y. Hidaka and R.D. Pisarski, Zero Point Energy of Renormalized Wilson Loops, Phys. Rev. D 80 (2009) 074504 [arXiv: 0907.4609] [INSPIRE].

[78] Y. Hidaka and R.D. Pisarski, Small shear viscosity in the semi quark gluon plasma, Phys. Rev. D 81 (2010) 076002 [arXiv: 0912.0940] [InSPIRE].

[79] A. Dumitru, Y. Guo, Y. Hidaka, C.P.K. Altes and R.D. Pisarski, How Wide is the Transition to Deconfinement?, Phys. Rev. D 83 (2011) 034022 [arXiv:1011.3820] [INSPIRE].

[80] A. Dumitru, Y. Guo, Y. Hidaka, C.P.K. Altes and R.D. Pisarski, Effective Matrix Model for Deconfinement in Pure Gauge Theories, Phys. Rev. D 86 (2012) 105017 [arXiv: 1205.0137] [INSPIRE].

[81] K. Kashiwa, R.D. Pisarski and V.V. Skokov, Critical endpoint for deconfinement in matrix and other effective models, Phys. Rev. D 85 (2012) 114029 [arXiv:1205.0545] [INSPIRE]. 
[82] R.D. Pisarski and V.V. Skokov, Gross-Witten-Wadia transition in a matrix model of deconfinement, Phys. Rev. D 86 (2012) 081701 [arXiv:1206.1329] [INSPIRE].

[83] K. Kashiwa and R.D. Pisarski, Roberge-Weiss transition and 't Hooft loops, Phys. Rev. D 87 (2013) 096009 [arXiv: 1301.5344] [INSPIRE].

[84] S. Lin, R.D. Pisarski and V.V. Skokov, Zero interface tension at the deconfining phase transition for a matrix model of a $\mathrm{SU}(\infty)$ gauge theory, Phys. Rev. D 87 (2013) 105002 [arXiv: 1301.7432] [INSPIRE].

[85] S. Lin, R.D. Pisarski and V.V. Skokov, Collisional energy loss above the critical temperature in QCD, Phys. Lett. B $\mathbf{7 3 0}$ (2014) 236 [arXiv:1312.3340] [INSPIRE].

[86] PHENIX collaboration, A. Adare et al., Observation of direct-photon collective flow in $\sqrt{s_{N N}}=200 \mathrm{GeV}$ Au + Au collisions, Phys. Rev. Lett. 109 (2012) 122302 [arXiv:1105.4126] [INSPIRE].

[87] ALICE collaboration, D. Lohner, Measurement of Direct-Photon Elliptic Flow in Pb-Pb Collisions at $\sqrt{s_{N N}}=2.76$ TeV, J. Phys. Conf. Ser. 446 (2013) 012028 [arXiv:1212.3995] [INSPIRE].

[88] E. Gava and R. Jengo, Perturbative Evaluation of the Thermal Wilson Loop, Phys. Lett. B 105 (1981) 285 [INSPIRE].

[89] Y. Burnier, M. Laine and M. Vepsäläinen, Dimensionally regularized Polyakov loop correlators in hot QCD, JHEP 01 (2010) 054 [Erratum ibid. 1301 (2013) 180] [arXiv:0911.3480] [INSPIRE].

[90] N. Brambilla, J. Ghiglieri, P. Petreczky and A. Vairo, The Polyakov loop and correlator of Polyakov loops at next-to-next-to-leading order, Phys. Rev. D 82 (2010) 074019 [arXiv: 1007.5172] [INSPIRE].

[91] A. Bazavov et al., Equation of state and QCD transition at finite temperature, Phys. Rev. D 80 (2009) 014504 [arXiv:0903.4379] [INSPIRE].

[92] C. DeTar and U.M. Heller, QCD Thermodynamics from the Lattice, Eur. Phys. J. A 41 (2009) 405 [arXiv: 0905.2949] [INSPIRE].

[93] Z. Fodor and S.D. Katz, The Phase diagram of quantum chromodynamics, arXiv:0908.3341 [INSPIRE].

[94] P. Petreczky, Lattice QCD at non-zero temperature, J. Phys. G 39 (2012) 093002 [arXiv: 1203.5320] [INSPIRE].

[95] S. Borsányi, Z. Fodor, C. Hölbling, S.D. Katz, S. Krieg and K.K. Szabo, Full result for the QCD equation of state with 2+1 flavors, Phys. Lett. B 730 (2014) 99 [arXiv:1309.5258] [INSPIRE].

[96] T. Bhattacharya et al., QCD Phase Transition with Chiral Quarks and Physical Quark Masses, Phys. Rev. Lett. 113 (2014) 082001 [arXiv:1402.5175] [InSPIRE].

[97] S. Sharma, QCD Thermodynamics on the Lattice, Adv. High Energy Phys. 2013 (2013) 452978 [arXiv: 1403.2102] [INSPIRE].

[98] C. Gale et al., Production and Elliptic Flow of Dileptons and Photons in a Matrix Model of the quark-gluon Plasma, Phys. Rev. Lett. 114 (2015) 072301 [arXiv:1409.4778] [INSPIRE].

[99] C.A. Islam, S. Majumder, N. Haque and M.G. Mustafa, Vector meson spectral function and dilepton production rate in a hot and dense medium within an effective QCD approach, JHEP 02 (2015) 011 [arXiv: 1411.6407] [INSPIRE]. 
[100] J.-P. Blaizot and E. Iancu, The Quark gluon plasma: Collective dynamics and hard thermal loops, Phys. Rept. 359 (2002) 355 [hep-ph/0101103] [INSPIRE].

[101] G. Baym, J.P. Blaizot, F. Gelis and T. Matsui, Landau-Pomeranchuck-Migdal effect in a quark-gluon plasma and the Boltzmann equation, Phys. Lett. B 644 (2007) 48 [hep-ph/0604209] [INSPIRE].

[102] E. Braaten, R.D. Pisarski and T.-C. Yuan, Production of Soft Dileptons in the quark-gluon Plasma, Phys. Rev. Lett. 64 (1990) 2242 [InSPIRE].

[103] M.A. Shifman, Quark hadron duality, hep-ph/0009131 [INSPIRE].

[104] A. Dumitru, R.D. Pisarski and D. Zschiesche, Dense quarks and the fermion sign problem, in a SU(N) matrix model, Phys. Rev. D 72 (2005) 065008 [hep-ph/0505256] [INSPIRE].

[105] D. Satow and W. Weise, Chiral symmetry breaking and confinement effects on dilepton and photon production around $T_{c}$, Phys. Rev. D 92 (2015) 056001 [arXiv:1505.03869] [INSPIRE].

[106] M. Le Bellac, Thermal Field Theory, Cambridge University Press, Cambridge, U.K. (1996).

[107] E. Wang and U.W. Heinz, A generalized fluctuation dissipation theorem for nonlinear response functions, Phys. Rev. D 66 (2002) 025008 [hep-th/9809016] [INSPIRE].

[108] P. Aurenche, F. Gelis and H. Zaraket, A Simple sum rule for the thermal gluon spectral function and applications, JHEP 05 (2002) 043 [hep-ph/0204146] [INSPIRE].

[109] P. Aurenche, F. Gelis, R. Kobes and H. Zaraket, Bremsstrahlung and photon production in thermal QCD, Phys. Rev. D 58 (1998) 085003 [hep-ph/9804224] [INSPIRE]. 